\title{
Fröhlich polaron and bipolaron: recent developments
}

\author{
Jozef T. Devreese* \\ Universiteit Antwerpen, Groenenborgerlaan 171, B-2020 Antwerpen, Belgium \\ Alexandre S. Alexandrovt \\ Department of Physics, Loughborough University, Loughborough LE11 3TU, \\ United Kingdom
}

\begin{abstract}
It is remarkable how the Fröhlich polaron, one of the simplest examples of a Quantum Field Theoretical problem, as it basically consists of a single fermion interacting with a scalar Bose field of ion displacements, has resisted full analytical or numerical solution at all coupling since $\sim 1950$, when its Hamiltonian was first written. The field has been a testing ground for analytical, semi-analytical, and numerical techniques, such as path integrals, strong-coupling perturbation expansion, advanced variational, exact diagonalisation (ED), and quantum Monte Carlo (QMC) techniques. This article reviews recent developments in the field of continuum and discrete (lattice) Fröhlich (bi)polarons starting with the basics and covering a number of active directions of research.
\end{abstract}

*Electronic address: jozef.devreese@ua.ac.be

${ }^{\dagger}$ Electronic address: a.s.alexandrov@lboro.ac.uk 


\section{Contents}

\section{INTRODUCTION}

\section{Continuum polaron}

A. Pekar's polaron

1. Ground state

2. Effective mass of Pekar's polaron

B. Weak-coupling Fröhlich polaron

C. Lee-Low-Pines transformation

D. All-coupling continuum polaron

1. Feynman theory

2. Diagrammatic Monte-Carlo algorithm

III. Response of continuum polarons

A. Mobility

B. Optical absorption at weak coupling

C. Optical absorption at strong coupling

D. Optical absorption at arbitrary coupling

E. Main-peak line and strong-coupling expansion

F. Comparison between optical conductivity spectra obtained by different methods

G. Sum rules for the optical conductivity spectra of Fröhlich polarons

H. Optical absorption spectra of continuum-polaron gas

I. Ripplopolarons

J. Polaron scaling relations

\section{Discrete Holstein and Fröhlich polaron}

A. Holstein model

1. Non-adiabatic Holstein polaron

2. Adiabatic Holstein polaron

B. Lang-Firsov canonical transformation

1. " $1 / \lambda "$ expansion and polaron band

2. Temperature effect on the polaron band 
C. Discrete Fröhlich polaron at strong coupling

D. Effect of dispersive phonons $\quad 65$

E. All-coupling discrete polaron $\quad 66$

1. Holstein model at any coupling $\quad 66$

2. Holstein polaron in infinite lattices $\quad 69$

3. Discrete Fröhlich polaron at any coupling $\quad 73$

F. Isotope effect on the polaron mass and the polaron band-structure 74

G. Jahn-Teller polaron

$\begin{array}{ll}\text { V. Response of discrete polarons } & 79\end{array}$
A. Hopping mobility
B. Optical conductivity
C. Spectral function of discrete strong-coupling polarons
D. Spectral function of discrete all-coupling polarons

\section{Bipolaron}

A. Polaron-polaron interaction $\quad 90$

B. Holstein bipolaron $\quad 92$

C. Continuum Fröhlich bipolaron $\quad 97$

D. Discrete strong-coupling Fröhlich bipolaron $\quad 100$

E. Discrete all-coupling Fröhlich bipolaron $\quad 105$

\begin{tabular}{ll} 
F. Polaronic exciton & 107 \\
\hline
\end{tabular}

VII. Current status of polarons and open problems 108

Acknowledgments

References

\section{INTRODUCTION}

Charge carriers in inorganic and organic matter interact with ion vibrations. The corresponding electron-phonon interaction (EPI) causes phase transformations, including superconductivity, and dominates the transport properties of many metals and semiconduc- 
tors. EPIs have been shown to be relevant in cuprate and other high-temperature superconductors through, for example, isotope substitution experiments (Khasanov et al. , 2004; Zhao and Morris , 1995; Zhao et al. , 1997), high resolution angle resolved photoemission (ARPES) (Gweon et al. , 2004; Lanzara et al. , 2001; Meevasana et al. , 2006), a number of earlier optical (Calvani et al. , 1994; Mihailović et al. , 1990; Zamboni et al. , 1989), neutron-scattering (Sendyka et al. , 1995) and more recent inelastic scattering (Reznik et al. , 2006), pump-probe (Gadermaier et al., 2009; Radovic et al. , 2008) and tunnelling (Shim et al. , 2008) measurements. In colossal magnetoresistance (CMR) manganites, isotope substitutions (Zhao et al. , 1996), X-ray and neutron scattering spectroscopies (Campbell et al., 2001, 2003) and a number of other experiments also show a significant effect of EPI on the physical properties (for review see (Tokura, 2000)). Therefore it has been suggested that the long-range (Fröhlich, 1954) and/or the moleculartype (e.g. (Jahn and Teller, 1937)) EPIs play significant role in high-temperature superconductors (see (Alexandrov, 1996; Alexandrov, 1998; Bednorz and Müller), 1988; Devreese, 1996, 2005; Müller, 2000), and references therein), and in CMR manganites (see (Alexandrov and Bratkovsky, 1999a; Edwards , 2002; Millis et al. ., 1995) and references therein). Very recent experimental observations of the optical conductivity in the Nb-doped $\mathrm{SrTiO}_{3}$ (van Mechelen et al., 2008) reveal the evidence of the mid-infrared optical conductivity band provided by the polaron mechanism like in many other oxides. The effective mass of the charge carriers is obtained by analyzing the Drude spectral weight. Defining the mass renormalization of the charge carriers as the ratio of the total electronic spectral weight and the Drude spectral weight, a twofold mass enhancement is obtained, which is attributed in Ref. (van Mechelen et al., 2008) to the electron-phonon coupling. The missing spectral weight is recovered according the sum rule (Devreese et al. , 1977) in a mid-infrared optical conductivity band. This band results from the electron-phonon coupling interaction, traditionally associated with the polaronic nature of the charge carriers. The effective mass obtained from the optical spectral weights yields an intermediate electron-phonon coupling strength, $3<\alpha<4$. Therefore it has been suggested in Ref. (van Mechelen et al., 2008) that the charge transport in the $\mathrm{Nb}$-doped $\mathrm{SrTiO}_{3}$ is carried by large polarons.

When EPI is sufficiently strong, electron Bloch states are affected even in the normal phase. Phonons are also affected by conduction electrons. In doped insulators bare phonons are well defined in insulating parent compounds, but microscopic separation of 
electrons and phonons is not so straightforward in metals and heavily doped insulators Maximov et al. , 1997), where the Born and Oppenheimer (1927) and density functional (Hohenberg and Kohn, 1964; Kohn and Sham, 1965) methods are used. Here we have to start with the first principle Hamiltonian describing conduction electrons and ions coupled by the Coulomb forces.

One cannot solve the corresponding Schrödinger equation perturbatively because the Coulomb interaction is strong. The ratio of the characteristic Coulomb energy to the kinetic energy is $r_{s}=m_{e} e^{2} /\left(4 \pi n_{e} / 3\right)^{1 / 3} \approx 1$ for the electron density $n_{e}=Z N=10^{23} \mathrm{~cm}^{-3}$ (here and further we take the volume of the system as $V=1$, unless specified otherwise, and $\left.\hbar=c=k_{B}=1\right)$. However, one can take advantage of the small value of the electron to ion mass ratio, $m_{e} / M<10^{-3}$. Ions are heavy and the amplitudes of their vibrations, $\langle|\mathbf{u}|\rangle \simeq \sqrt{1 / M \omega_{D}}$, near equilibrium positions are much smaller than the lattice constant $\left(a=N^{-1 / 3}\right),\langle|\mathbf{u}|\rangle / a \approx\left(m_{e} / M r_{s}\right)^{1 / 4} \ll 1$. In this estimate we take the characteristic vibration frequency $\omega_{D}$ of the order of the ion plasma frequency $\omega_{0}=\sqrt{4 \pi N Z^{2} e^{2} / M}$. Hence one can expand the Hamiltonian in powers of $|\mathbf{u}|$.

Any further progress requires a simplifying physical idea, which commonly is to approach the ground state of the many-electron system via a one-electron picture. In the framework of the local density approximation (LDA), where the Coulomb electron-electron interaction is replaced by an effective one-body potential, the Hamiltonian is written as

$$
H=H_{e}+H_{p h}+H_{e-p h}+H_{e-e}
$$

where

$$
\begin{aligned}
H_{e} & =\sum_{\mathbf{k}, n, s} \xi_{n \mathbf{k} s} c_{n \mathbf{k} s}^{\dagger} c_{n \mathbf{k} s}, \\
H_{p h} & =\sum_{\mathbf{q}, \nu} \omega_{\mathbf{q} \nu}\left(d_{\mathbf{q} \nu}^{\dagger} d_{\mathbf{q} \nu}+1 / 2\right)
\end{aligned}
$$

describe independent Bloch electrons and phonons, created (annihilated) by $c_{n \mathbf{k} s}^{\dagger}\left(c_{n \mathbf{k} s}\right)$ and by $d_{\mathbf{q} \nu}^{\dagger}\left(d_{\mathbf{q} \nu}\right)$, respectively, $\xi_{n \mathbf{k} s}=E_{n \mathbf{k} s}-\mu$ is the band energy spectrum with respect to the chemical potential $\mu,(\mathbf{k}, \mathbf{q})$ are quasi-momenta of electrons and phonons, respectively, $n$ is the electron band index, $\nu$ is the phonon mode index, and $s$ is the electron spin. The part of the electron-phonon interaction, which is linear in the phonon operators, is written as

$$
H_{e-p h}=\frac{1}{\sqrt{2 N}} \sum_{\mathbf{k}, \mathbf{q}, n, n^{\prime}, \nu, s} \gamma_{n n^{\prime}}(\mathbf{q}, \mathbf{k}, \nu) \omega_{\mathbf{q} \nu} c_{n^{\prime} \mathbf{k} s}^{\dagger} c_{n \mathbf{k}-\mathbf{q} s} d_{\mathbf{q} \nu}+H . c .,
$$


where $\gamma_{n n^{\prime}}(\mathbf{q}, \mathbf{k}, \nu)$ is the dimensionless matrix element. If we restrict the summations over $\mathbf{q}$ and $\mathbf{k}$ to the first Brillouin zone of the crystal, then $H_{e-p h}$ should also include the summation over reciprocal lattice vectors $\mathbf{G}$ of umklapp scattering contributions where $\mathbf{q}$ is replaced by $\mathbf{q}+\mathbf{G}$. The terms of $H_{e-p h}$ which are quadratic and of higher orders in the phonon operators, $d_{\mathbf{q} \nu}$, are usually small. They play a role only for those phonons which are not coupled with electrons by the linear interaction, Eq.(44).

The electron-electron correlation energy of a homogeneous electron system is often written as

$$
H_{e-e}=\frac{1}{2} \sum_{\mathbf{q}} V_{c}(\mathbf{q}) \rho_{\mathbf{q}}^{\dagger} \rho_{\mathbf{q}}
$$

where $V_{c}(\mathbf{q})$ is a matrix element, which is zero for $\mathbf{q}=0$ because of electroneutrality and $\rho_{\mathbf{q}}^{\dagger}=\sum_{\mathbf{k}, s} c_{\mathbf{k} s}^{\dagger} c_{\mathbf{k}+\mathbf{q} s}$ is the density fluctuation operator. $H$ should also include a random potential in doped semiconductors and amorphous metals, which could affect the EPI matrix element (Belitz and Kirkpatrick, 1994).

For the purpose of this review we mostly confine our discussions to a single band approximation with the EPI matrix element $\gamma_{n n}(\mathbf{q}, \mathbf{k}, \nu)=\gamma(\mathbf{q})$ depending only on the momentum transfer $\mathbf{q}$. The approximation allows for qualitative and in many cases quantitative descriptions of essential polaronic effects in advanced materials. Nevertheless there are might be degenerate atomic orbitals in solids coupled to local molecular-type Jahn-Teller distortions, where one has to consider multi-band electron energy structures.

Quantitative calculations of the matrix element in the whole region of momenta can be performed from pseudopotentials (Baryakhtar et al. , 1999; Maximov et al. , 1997). On the other hand one can parameterize EPI rather than to compute it from first principles in many physically important cases (Mahan, 1990). There are three most important interactions in doped semiconductors, which are polar coupling to optical phonons (the Fröhlich EPI), deformation potential coupling to acoustical phonons, and the local (Holstein) EPI with molecular type vibrations in complex lattices. While the matrix element is ill defined in metals, the bare phonons $\omega_{\mathbf{q} \nu}$ and the electron band structure $E_{n \mathbf{k} s}$ are well defined in doped semiconductors, which have their parent dielectric compounds. Here the effect of carriers on the crystal field and on the dynamic matrix is small while the carrier density is much less than the atomic one. Hence one can use the band structure and the crystal field of parent insulators to calculate the matrix element in doped semiconductors. The 
interaction constant $\gamma(\mathbf{q})$ has different $q$-dependence for different phonon branches. In the long wavelength limit $(q \ll \pi / a), \gamma(\mathbf{q}) \propto q^{n}$, where $n=-1,0$ and $n=-1 / 2$ for polar optical, molecular $\left.\left(\omega_{\mathbf{q}}=\omega_{0}\right)\right)$ and acoustic $\left(\omega_{\mathbf{q}} \propto q\right)$ phonons, respectively. Not only $q$ dependence is known but also the absolute values of $\gamma(\mathbf{q})$ are well parameterized in this limit. For example in polar semiconductors $|\gamma(\mathbf{q})|^{2}=4 \pi e^{2} / \kappa \omega_{0} q^{2}$, where $\kappa=\left(\varepsilon^{-1}-\varepsilon_{0}^{-1}\right)^{-1}$, and $\varepsilon$ and $\varepsilon_{0}$ are high-frequency and static dielectric constants, respectively. If the crystal lacks an inversion center to be piezoelectric, there is EPI with piezoelectric (acoustic) phonons with an anysotropic matrix element, which also contribute to a polaron effect and a Coulomb-like attraction of two polarons (Mahan, 1972).

To get a better insight into physical constraints of the above approximation let us transform the Bloch states to the real space or Wannier states using the canonical linear transformation of the electron operators, $c_{i}=N^{-1} \sum_{\mathbf{k}} e^{i \mathbf{k} \cdot \mathbf{m}_{C}} c_{\mathbf{k} s}$, where $i=(\mathbf{m}, s)$ includes both site $\mathbf{m}$ and spin quantum numbers. In this site (Wannier) representation the electron kinetic energy takes the following form

$$
H_{e}=\sum_{i, j} t(\mathbf{m}-\mathbf{n}) \delta_{s s^{\prime}} c_{i}^{\dagger} c_{j}
$$

where $t(\mathbf{m})=N^{-1} \sum_{\mathbf{k}} E_{\mathbf{k}} e^{i \mathbf{k} \cdot \mathbf{m}}$ is the "bare" hopping integral, $j=\left(\mathbf{n}, s^{\prime}\right)$, and $E_{\mathbf{k}}$ is the Bloch band dispersion in the rigid lattice.

The electron-phonon interaction and the Coulomb correlations acquire simple forms in the Wannier representation, if their matrix elements in the momentum representation depend only on the momentum transfer q (here we follow (Alexandrov and Mott, 1995)),

$$
\begin{gathered}
H_{e-p h}=\sum_{\mathbf{q}, i} \omega_{\mathbf{q}} \hat{n}_{i}\left[u_{i}(\mathbf{q},) d_{\mathbf{q}}+H . c .\right] \\
H_{e-e}=\frac{1}{2} \sum_{i \neq j} V_{c}(\mathbf{m}-\mathbf{n}) \hat{n}_{i} \hat{n}_{j}
\end{gathered}
$$

where

$$
u_{i}(\mathbf{q})=\frac{1}{\sqrt{2 N}} \gamma(\mathbf{q}) e^{i \mathbf{q} \cdot \mathbf{m}}
$$

and

$$
V_{c}(\mathbf{m})=\frac{1}{N} \sum_{\mathbf{q}} V_{c}(\mathbf{q}) e^{i \mathbf{q} \cdot \mathbf{m}},
$$

are the matrix elements of the electron-phonon and Coulomb interactions, respectively, in the Wannier representation for electrons, and $\hat{n}_{i}=c_{i}^{\dagger} c_{i}$ is the density operator. 
We see that taking the interaction matrix element depending only on the momentum transfer one neglects the terms in the electron-phonon and Coulomb interactions, which are proportional to the overlap integrals of the Wannier orbitals on different sites. This approximation is justified for narrow band materials, where the electron bandwidth is less than the characteristic magnitude of the crystal field potential. In the Wannier representation the Hamiltonian is

$$
\begin{aligned}
H= & \sum_{i, j} t(\mathbf{m}-\mathbf{n}) \delta_{s s^{\prime}} c_{i}^{\dagger} c_{j}+\sum_{\mathbf{q}, i} \omega_{\mathbf{q}} \hat{n}_{i}\left[u_{i}(\mathbf{q}) d_{\mathbf{q}}+H . c .\right] \\
& +\frac{1}{2} \sum_{i \neq j} V_{c}(\mathbf{m}-\mathbf{n}) \hat{n}_{i} \hat{n}_{j}+\sum_{\mathbf{q}} \omega_{\mathbf{q}}\left(d_{\mathbf{q}}^{\dagger} d_{\mathbf{q}}+1 / 2\right) .
\end{aligned}
$$

One can transform it further using the site-representation also for phonons. The site representation of $H_{e-p h}$ is particularly convenient for the interaction with dispersionless modes, when $\omega_{\mathbf{q}}=\omega_{0}$ and the phonon polarization vector $\mathbf{e}_{\mathbf{q}}=\mathbf{e}$ are roughly $q$-independent. Introducing the phonon site-operators $d_{\mathbf{n}}=N^{-1} \sum_{\mathbf{q}} e^{i \mathbf{q} \cdot \mathbf{n}} d_{\mathbf{q}}$ one obtains in this case,

$$
H_{e-p h}=\omega_{0} \sum_{\mathbf{n}, \mathbf{m}, s} g(\mathbf{m}-\mathbf{n})\left(\mathbf{e} \cdot \mathbf{e}_{\mathbf{m}-\mathbf{n}}\right) \hat{n}_{\mathbf{m} s}\left(d_{\mathbf{n}}^{\dagger}+d_{\mathbf{n}}\right),
$$

where $g(\mathbf{m})$ is the dimensionless force acting between the electron on site $\mathbf{m}$ and the displacement of ion $\mathbf{n}$, proportional to the Fourier transform of $\gamma(\mathbf{q})$, and $\mathbf{e}_{\mathbf{m}-\mathbf{n}} \equiv(\mathbf{m}-\mathbf{n}) /|\mathbf{m}-\mathbf{n}|$ is the unit vector in the direction from the electron on site $\mathbf{m}$ to the ion $\mathbf{n}$. The real space representation is particularly convenient in parameterizing EPI in complex lattices. Atomic orbitals of an ion adiabatically follow its motion. Therefore the electron does not interact with the displacement of the ion, whose orbitals it occupies, that is $g(0)=0$.

\section{CONTINUUM POLARON}

If characteristic phonon frequencies are sufficiently low, the local deformation of ions, caused by electron itself, creates a potential well, which traps the electron even in a perfect crystal lattice. This self-trapping phenomenon was predicted by Landau (Landau, 1933) more than 70 years ago. It was studied in greater detail (Devreese, 1996; Feynman, 1955; Fröhlich, 1954; Pekar, 1946; Rashba , 1957) in the effective mass approximation for the electron placed in a continuum polarizible (or deformable) medium, which leads to a socalled large or continuum polaron. Large polaron wave functions and corresponding lattice 
distortions spread over many lattice sites. The self-trapping is never complete in the perfect lattice. Due to finite phonon frequencies ion polarisations can follow polaron motion if the motion is sufficiently slow. Hence, large polarons with a low kinetic energy propagate through the lattice as free electrons but with an enhanced effective mass.

When the polaron binding energy $E_{p}$ is larger than the halfbandwidth $D$ of the electron band, all states in the Bloch bands are "dressed" by phonons. In this strong-coupling regime, $\lambda=E_{p} / D>1$, the finite bandwidth becomes important, so the continuum approximation cannot be applied. In this case the carriers are described as small or discrete (lattice) polarons. The main features of small polarons were understood a long time ago (Eagles , 1963; Holstein , 1959a,b; Lang and Firsov, 1962; Sewell, 1958; Tyablikov, 1952; Yamashita and Kurosawa, 1958). The first identification of small polarons in solids was made for non-stoichiometric uranium dioxide in Refs. (Devreese, 1963; Nagels et al. 1963). Large and small polarons were discussed in a number of review papers and textbooks, for example (Alexandrov and Mott, 1994, 1995; Appel , 1968; Böttger and Brvksin , 1985; Devreese, 1996; Firsov , 1975; Itoh and Stoneham , 2001; Mahan , 1990; Mitra et al., 1987; Rashba, 2005; Salje et al. , 1995).

In many models of EPI the ground-state polaron energy is an analytical function of the coupling constant for any dimensionality of space (Fehske and Trugman), 2007; Gerlach and Löwen, 1991; Hague et al. , 2006a; Löwen, 1988; Peeters and Devreese, 1982). There is no abrupt (nonanalytical) phase transition of the ground state as the electron-phonon coupling increases. It is instead a crossover from Bloch states of band electrons or large polarons propagating with almost bare mass in a rigid lattice to heavily dressed Bloch states of small polarons propagating at low temperatures with an exponentially enhanced effective mass. The ground-state wave function of any polaron is delocalized for any coupling strength. This result holds for both finite-site models and infinite-site models (Löwen, 1988).

\section{A. Pekar's polaron}

First let us briefly discuss a single electron interacting with the lattice deformation in the continuum approximation, as studied by Pekar (1946). In his model a free electron interacts with the dielectric polarisable continuum, described by the static $\varepsilon_{0}$ and the optical 
(high frequency) $\varepsilon$ dielectric constants. This is the case for carriers interacting with optical phonons in ionic crystals under the condition that the size of the self-trapped state is large compared with the lattice constant so that the lattice discreteness is irrelevant.

\section{Ground state}

Describing the ionic crystal as a polarisable dielectric continuum one should keep in mind that only the ionic part of the total polarisation contributes to the polaron state. The interaction of a carrier with valence electrons responsible for the optical properties is taken into account via the Hartree-Fock periodic potential and included in the band mass $m$. Therefore only ion displacements contribute to the self-trapping. Following Pekar we minimise the sum $E(\psi)$ of the electron kinetic energy and the potential energy due to the self-induced polarisation field (here we follow (Alexandrov and Mott , 1995)),

$$
E(\psi)=\int d \mathbf{r}\left[\psi^{*}(\mathbf{r})\left(-\frac{\nabla^{2}}{2 m}\right) \psi(\mathbf{r})-\mathbf{P}(\mathbf{r}) \cdot \mathbf{D}(\mathbf{r})\right]
$$

where

$$
\mathbf{D}(\mathbf{r})=e \nabla \int d \mathbf{r}^{\prime} \frac{\left|\psi\left(\mathbf{r}^{\prime}\right)\right|^{2}}{\left|\mathbf{r}-\mathbf{r}^{\prime}\right|}
$$

is the electric field of the electron in the state with the wave function $\psi(\mathbf{r})$ and $\mathbf{P}$ is the ionic part of the lattice polarisation. Minimising $E(\psi)$ with respect to $\psi^{*}(\mathbf{r})$ at fixed $\mathbf{P}$ and $\int d \mathbf{r}|\psi(\mathbf{r})|^{2}=1$ one arrives at the equation of motion,

$$
\left(-\frac{\nabla^{2}}{2 m}-e \int d \mathbf{r}^{\prime} \mathbf{P}\left(\mathbf{r}^{\prime}\right) \cdot \nabla^{\prime} \frac{1}{\left|\mathbf{r}^{\prime}-\mathbf{r}\right|}\right) \psi(\mathbf{r})=E_{0} \psi(\mathbf{r}),
$$

where $E_{0}$ is the polaron ground state energy. The ionic part of the total polarisation is obtained using the definition of the susceptibilities $\chi_{0}$ and $\chi, \mathbf{P}=\left(\chi_{0}-\chi\right) \mathbf{D}$. The dielectric susceptibilities $\chi_{0}$ and $\chi$ are related to the static and high frequency dielectric constants, respectively, $\left(\chi_{0}=\left(\varepsilon_{0}-1\right) / 4 \pi \varepsilon_{0}\right.$, and $\left.\chi=(\varepsilon-1) / 4 \pi \varepsilon\right)$, so that $\mathbf{P}=\frac{\mathbf{D}}{4 \pi \kappa}$. Then the equation of motion becomes

$$
\left(-\frac{\nabla^{2}}{2 m}-\frac{e^{2}}{4 \pi \kappa} \int d \mathbf{r}^{\prime} \int d \mathbf{r}^{\prime \prime}\left|\psi\left(\mathbf{r}^{\prime \prime}\right)\right|^{2} \nabla^{\prime} \frac{1}{\left|\mathbf{r}^{\prime}-\mathbf{r}^{\prime \prime}\right|} \cdot \nabla^{\prime} \frac{1}{\left|\mathbf{r}^{\prime}-\mathbf{r}\right|}\right) \psi(\mathbf{r})=E_{0} \psi(\mathbf{r}) .
$$

Differentiating by parts with the use of $\nabla^{2} r^{-1}=-4 \pi \delta(\mathbf{r})$ one obtains

$$
\left(-\frac{\nabla^{2}}{2 m}-\frac{e^{2}}{\kappa} \int d \mathbf{r}^{\prime} \frac{\left|\psi\left(\mathbf{r}^{\prime}\right)\right|^{2}}{\left|\mathbf{r}^{\prime}-\mathbf{r}\right|}\right) \psi(\mathbf{r})=E_{0} \psi(\mathbf{r})
$$


The solution of this nonlinear integro-differential equation can be found using a variational minimisation of the functional

$$
J(\psi)=\frac{1}{2 m} \int d \mathbf{r}|\nabla \psi(\mathbf{r})|^{2}-\frac{1}{2 m a_{B}} \int d \mathbf{r} d \mathbf{r}^{\prime} \frac{|\psi(\mathbf{r})|^{2}\left|\psi\left(\mathbf{r}^{\prime}\right)\right|^{2}}{\left|\mathbf{r}^{\prime}-\mathbf{r}\right|},
$$

where $a_{B}=\kappa / m e^{2}$ is the effective Bohr radius. The simplest choice of the normalised trial function is $\psi(\mathbf{r})=A e^{-r / r_{p}}$ with $A=\left(\pi r_{p}^{3}\right)^{-1 / 2}$. Substituting the trial function into the functional yields $J(\psi)=T+\frac{1}{2} U$, where the kinetic energy is $T=1 / 2 m r_{p}^{2}$, and the potential energy $U=-5 / 8 m a_{B} r_{p}$. Minimising the functional with respect to $r_{p}$ yields the polaron radius, $r_{p}=16 a_{B} / 5$, and the ground state energy $E_{0}=T+U$ as $E_{0}=-0.146 / m a_{B}^{2}$. This can be compared with the ground state energy of the hydrogen atom $-0.5 m_{e} e^{4}$, where $m_{e}$ is the free electron mass. Their ratio is $0.3 \mathrm{~m} /\left(m_{e} \kappa^{2}\right)$. In typical polar solids $\kappa \gtrsim 4$, so that the continuum polaron binding energy is about $0.25 \mathrm{eV}$ or less, if $m \simeq m_{e}$. The potential energy in the ground state is $U=-4 T=4 E_{0} / 3$.

The lowest photon energy $\omega_{\text {min }}$ to excite the polaron into the bare electron band is $\omega_{\min }=\left|E_{0}\right|$. The ion configuration does not change in the photoexcitation process of the polaron. However, a lower activation energy $W_{T}$ is necessary, if the self-trapped state disappears together with the polarisation well due to thermal fluctuations, $W_{T}=\left|E_{0}\right|-U_{d}$, where $U_{d}$ is the deformation energy. In ionic crystals

$$
U_{d}=\frac{1}{2} \int d \mathbf{r} \mathbf{P}(\mathbf{r}) \cdot \mathbf{D}(\mathbf{r})
$$

which for the ground state is $U_{d}=2\left|E_{0}\right| / 3$. Therefore the thermal activation energy is $W_{T}=\left|E_{0}\right| / 3$. The ratio of four characteristic energies for the continuum Pekar's polaron is $W_{T}: U_{d}: \omega_{\text {min }}:|U|=1: 2: 3: 4$ (Pekar, 1951).

Using Pekar's choice,

$$
\psi(r)=A\left(1+r / r_{p}+\beta r^{2}\right) e^{-r / r_{p}}
$$

one obtains $A=0.12 / r_{p}^{3 / 2}, \beta=0.45 / r_{p}^{2}$, the polaron radius $r_{p}=1.51 a_{B}$ and a better estimate for the ground state energy, $E_{0}=-0.164 / m a_{B}^{2}$ as compared to the result of the simplest exponential choice.

\section{Effective mass of Pekar's polaron}

The lattice polarization is responsible for the polaron mass enhancement (Landau and Pekar, 1948). Within the continuum approximation the evolution of 
the lattice polarisation $\mathbf{P}(\mathbf{r}, t)$ is described by a harmonic oscillator subjected to an external force $\sim \mathbf{D} / \kappa:$

$$
\omega_{0}^{-2} \frac{\partial^{2} \mathbf{P}(\mathbf{r}, t)}{\partial t^{2}}+\mathbf{P}(\mathbf{r}, t)=\frac{\mathbf{D}(\mathbf{r}, t)}{4 \pi \kappa},
$$

where $\omega_{0}$ is the optical phonon frequency. If during the characteristic time of the lattice relaxation $\simeq \omega_{0}^{-1}$ the polaron moves a distance much less than the polaron radius, the polarisation practically follows the polaron motion. Hence for a slow motion with the velocity $v \ll \omega_{0} a_{B}$ the first term in Eq.(21) is a small perturbation, so that

$$
\mathbf{P}(\mathbf{r}, t) \approx \frac{1}{4 \pi \kappa}\left(\mathbf{D}(\mathbf{r}, t)-\omega_{0}^{-2} \frac{\partial^{2} \mathbf{D}(\mathbf{r}, t)}{\partial t^{2}}\right)
$$

The total energy of the crystal with an extra electron,

$$
E=E(\psi)+2 \pi \kappa \int d \mathbf{r}\left[\mathbf{P}(\mathbf{r}, \mathbf{t})^{2}+\omega_{0}^{-2}\left(\frac{\partial \mathbf{P}(\mathbf{r}, t)}{\partial t}\right)^{2}\right],
$$

is determined in such a way that it gives Eq.(21) when it is minimised with respect to $\mathbf{P}$. We note that the first term of the lattice contribution to $E$ is the deformation energy $U_{d}$, discussed in the previous section. The lattice part of the total energy depends on the polaron velocity and contributes to the effective mass. Replacing the static wave function $\psi(\mathbf{r})$ in all expressions for $\psi(\mathbf{r}-\mathbf{v} t)$ and neglecting a contribution to the total energy of higher orders than $v^{2}$ one obtains

$$
E=E_{0}+U_{d}+\frac{m^{*} v^{2}}{2}
$$

where

$$
m^{*}=-\frac{1}{12 \pi \omega_{0}^{2} \kappa} \int d \mathbf{r} \mathbf{D}(\mathbf{r}) \cdot \nabla^{2} \mathbf{D}(\mathbf{r})
$$

is the polaron mass. Using the equation $\nabla \cdot \mathbf{D}=-4 \pi e|\psi(\mathbf{r})|^{2}$ yields

$$
m^{*}=\frac{4 \pi e^{2}}{3 \omega_{0}^{2} \kappa} \int d \mathbf{r}|\psi(\mathbf{r})|^{4}
$$

Calculating the integral with the trial function Eq.(20) one obtains

$$
m^{*} \approx 0.02 \alpha^{4} m
$$

where $\alpha$ is the dimensionless constant, defined as $\alpha=\left(e^{2} / \kappa\right) \sqrt{m / 2 \omega_{0}}$.

Concluding the discussion of the Pekar's polarons let us specify conditions of its existence. The polaron radius should be large compared with the lattice constant, $r_{p} \gg a$ to justify the effective mass approximation for the electron. Hence the value of the coupling constant $\alpha$ 
should not be very large, $\alpha \ll\left(D / z \omega_{0}\right)^{1 / 2}$, where $D \simeq z / 2 m a^{2}$ is the bare half-bandwidth, and $z$ is the lattice coordination number. On the other hand the classical approximation for the lattice polarisation is justified if the number of phonons taking part in the polaron cloud is large. This number is of the order of $U_{d} / \omega_{0}$. The total energy of the immobile polaron and the deformed lattice is $E=-0.109 \alpha^{2} \omega_{0}$ and $U_{d}=0.218 \alpha^{2} \omega_{0}$, respectively. Then $\alpha$ is bounded from below by the condition $U_{d} / \omega_{0} \gg 1$ as $\alpha^{2} \gg 5$. The typical adiabatic ratio $D / \omega_{0}$ is of the order of 10 to 100 . In fact in many transition metal oxides with narrow bands and high optical phonon frequencies this ratio is about 10 or even less, which makes the continuum strong-coupling polaron hard to be realised in oxides and related ionic compounds with light ions (Alexandrov and Mott, 1995).

\section{B. Weak-coupling Fröhlich polaron}

Fröhlich (1954) applied the second quantisation form of the electron-lattice interaction (Fröhlich et al., 1950$)$ to describe the large polaron in the weak-coupling regime, $\alpha<1$, where the quantum nature of lattice polarisation becomes important,

$$
H=-\frac{\nabla^{2}}{2 m}+\sum_{\mathbf{q}}\left(V_{\mathbf{q}} d_{\mathbf{q}} e^{i \mathbf{q} \cdot \mathbf{r}}+h . c .\right)+\sum_{\mathbf{q}} \omega_{\mathbf{q}}\left(d_{\mathbf{q}}^{\dagger} d_{\mathbf{q}}+1 / 2\right) .
$$

The quantum states of the noninteracting electron and phonons are specified by the electron momentum $\mathbf{k}$ and the phonon occupation number $\left\langle d_{\mathbf{q}}^{\dagger} d_{\mathbf{q}}\right\rangle \equiv n_{\mathbf{q}}=0,1,2, \ldots \infty$. At zero temperature the unperturbed state is the vacuum, $|0\rangle$, of phonons and the electron plane wave

$$
|\mathbf{k}, 0\rangle=e^{i \mathbf{k} \cdot \mathbf{r}}|0\rangle
$$

While the coupling is weak one can apply the perturbation theory. The interaction couples the state Eq.(29) with the energy $k^{2} / 2 m$ and the state with the energy $(\mathbf{k}-\mathbf{q})^{2} / 2 m+\omega_{0}$ of a single phonon with momentum $\mathbf{q}$ and the electron with momentum $\mathbf{k}-\mathbf{q},\left|\mathbf{k}-\mathbf{q}, 1_{\mathbf{q}}\right\rangle=$ $e^{i(\mathbf{k}-\mathbf{q}) \cdot \mathbf{r}}\left|1_{\mathbf{q}}\right\rangle$. The corresponding matrix element is $\left\langle\mathbf{k}-\mathbf{q}, 1_{\mathbf{q}}\left|H_{e-p h}\right| \mathbf{k}, 0\right\rangle=V_{\mathbf{q}}^{*}$. There is no diagonal matrix elements of $H_{e-p h}$, so the second order energy $\tilde{E}_{\mathbf{k}}$ is

$$
\tilde{E}_{\mathbf{k}}=\frac{k^{2}}{2 m}-\sum_{\mathbf{q}} \frac{\left|V_{\mathbf{q}}\right|^{2}}{(\mathbf{k}-\mathbf{q})^{2} / 2 m+\omega_{0}-k^{2} / 2 m} .
$$

There is no imaginary part of $\tilde{E}_{\mathbf{k}}$ for a slow electron with $k<q_{p}$, where

$$
q_{p}=\min \left(m \omega_{0} / q+q / 2\right)=\left(2 m \omega_{0}\right)^{1 / 2},
$$


which means that the momentum is conserved. Evaluating the integrals one arrives at

$$
\tilde{E}_{\mathbf{k}}=\frac{k^{2}}{2 m}-\frac{\alpha \omega_{0} q_{p}}{k} \arcsin \left(\frac{k}{q_{p}}\right)
$$

which for a very slow motion $k \ll q_{p}$ yields

$$
\tilde{E}_{\mathrm{k}} \simeq-\alpha \omega_{0}+\frac{k^{2}}{2 m^{*}} .
$$

Here the first term is minus the polaron binding energy, $-E_{p}$. The effective mass of the polaron is enhanced as

$$
m^{*}=\frac{m}{1-\alpha / 6} \simeq m(1+\alpha / 6)
$$

due to a phonon "cloud" accompanying the slow polaron. The number of virtual phonons $N_{p h}$ in the cloud is given by taking the expectation value of the phonon number operator, $N_{p h}=\left\langle\sum_{\mathbf{q}} d_{\mathbf{q}}^{\dagger} d_{\mathbf{q}}\right\rangle$, where bra and ket refer to the perturbed state,

$$
|\rangle=|0\rangle+\sum_{\mathbf{q}^{\prime}} \frac{V_{\mathbf{q}^{\prime}}^{*}}{k^{2} / 2 m-\left(\mathbf{k}-\mathbf{q}^{\prime}\right)^{2} / 2 m-\omega_{0}}\left|1_{\mathbf{q}^{\prime}}\right\rangle .
$$

For the polaron at rest $(\mathbf{k}=0)$ one obtains $N_{p h}=\alpha / 2$. Hence the Fröhlich coupling constant, $\alpha$, measures the cloud 'thickness'. One can also calculate the lattice charge density induced by the electron. The electrostatic potential $e \phi(\mathbf{r})$ is given by the average of the interaction term of the Hamiltonian

$$
e \phi(\mathbf{r})=\left\langle\sum_{\mathbf{q}} V_{\mathbf{q}} e^{i \mathbf{q} \cdot \mathbf{r}} d_{\mathbf{q}}+H . c .\right\rangle,
$$

and the charge density $\rho(\mathbf{r})$ is related to the electrostatic potential by Poisson's equation $\nabla \phi=-4 \pi \rho$. Using these equations one obtains

$$
\rho(\mathbf{r})=-\frac{1}{2 \pi e} \sum_{\mathbf{q}} \frac{q^{2}\left|V_{\mathbf{q}}\right|^{2} \cos (\mathbf{q} \cdot \mathbf{r})}{\omega_{0}+q^{2} / 2 m}
$$

which yields

$$
\rho(\mathbf{r})=-\frac{e q_{p}^{3}}{4 \pi \kappa} \frac{e^{-q_{p} r}}{q_{p} r} .
$$

The mean extension of the phonon cloud, which can be taken as the radius of the weak coupling polaron, is $r_{p}=q_{p}^{-1}$, and the total induced charge is $Q=\int d \mathbf{r} \rho(\mathbf{r})=-e / \kappa$. 


\section{Lee-Low-Pines transformation}

One can put the Fröhlich result on a variational basis by applying the Lee-Low-Pines (LLP) transformation (Lee et al. , 1953), which removes the electron coordinate, followed by the displacement transformation (Gurari, 1953; Lee and Pines, 1952; Tyablikov, 1952). The latter serves to account for that part of the lattice polarisation which follows the electron instantaneously. The remaining part of the polarisation field turns out to be small, if the coupling constant is not extremely large. In the opposite extreme limit, which is Pekar's strong-coupling regime discussed above, one can construct the perturbation theory by an expansion in descending powers of $\alpha$ (Allcock, 1963; Bogoliubov, 1950; Bogoliubov Jr. , 1994). Alternatively one can apply Feynman's path-integral formalism to remove the phonon field at the expense of a non-instantaneous interaction of electron with itself (II.D), and also (Schultz, 1962)).

The transformation can be written as

$$
|\tilde{N}\rangle=\exp (S)|N\rangle
$$

where $S$ is an anti-Hermitian operator: $S^{+}=-S$. In our case $|N\rangle$ is a single-electron multiphonon wave function. The transformed eigenstate, $|\tilde{N}\rangle$ satisfies the Schrödinger equation, $\tilde{H}|\tilde{N}>=E| \tilde{N}\rangle$, with the transformed Hamiltonian

$$
\tilde{H}=\exp (S) H \exp (-S)
$$

If all operators are transformed according to Eq.(40) the physical averages remain unchanged. LLP transformation eliminating the electron coordinate in the Hamiltonian is defined as

$$
S_{L L P}=i \sum_{\mathbf{q}}(\mathbf{q} \cdot \mathbf{r}) d_{\mathbf{q}}^{\dagger} d_{\mathbf{q}}
$$

The transformed Hamiltonian is obtained as

$$
\tilde{H}=\frac{1}{2 m}\left(-i \nabla-\sum_{\mathbf{q}} \mathbf{q} d_{\mathbf{q}}^{\dagger} d_{\mathbf{q}}\right)^{2}+\sum_{\mathbf{q}}\left(V_{\mathbf{q}} d_{\mathbf{q}}+H . c .\right)+\omega_{0} \sum_{\mathbf{q}}\left(d_{\mathbf{q}}^{\dagger} d_{\mathbf{q}}+1 / 2\right) .
$$

The electron coordinate is absent in $\tilde{H}$. Hence the eigenstates $|\tilde{N}\rangle$ are classified with the momentum $\mathbf{K}$, which is the conserving total momentum of the system, $|\tilde{N}\rangle=e^{i \mathbf{K} \cdot \mathbf{r}}\left|\tilde{N}_{p h}\right\rangle$, where $\left|\tilde{N}_{p h}\right\rangle$ is an eigenstate of phonons. The number of virtual phonons is not small in the 
intermediate coupling regime. Therefore one cannot apply the perturbation theory for $\tilde{H}$. However, one can remove the essential part of the interaction term in the Hamiltonian by the displacement canonical transformation,

$$
S=\sum_{\mathbf{q}} f(\mathbf{q}) d_{\mathbf{q}}-H . c .,
$$

where $c$-number $f(\mathbf{q})$ is determined by minimisation of the ground state energy. Assuming that the transformed ground state is the phonon vacuum $e^{S}\left|\tilde{N}_{p h}\right\rangle=|0\rangle$, one obtains the energy $E_{\mathbf{K}}$ as

$$
E_{\mathbf{K}}=\frac{\left(1-\eta^{2}\right) K^{2}}{2 m}-\frac{\alpha \omega_{0} q_{p}}{K(1-\eta)} \sin ^{-1}\left(\frac{K(1-\eta)}{q_{p}}\right),
$$

where

$$
\eta(1-\eta)^{2}=\frac{\alpha q_{p}^{3}}{2 K^{3}}\left(\frac{(1-\eta) K}{\sqrt{q_{p}^{2}-(1-\eta)^{2} K^{2}}}-\sin ^{-1} \frac{(1-\eta) K}{q_{p}}\right) .
$$

Only the term independent of $K$ needs to be retained in $\eta$ for a slow polaron with $K \ll q_{p}$,

$$
\eta=\frac{\alpha / 6}{1+\alpha / 6}
$$

Then the energy up to the second order in $K$ is given by

$$
E_{\mathbf{K}}=-\alpha \omega_{0}+\frac{K^{2}}{2 m^{*}}
$$

where the polaron mass is $m^{*}=m(1+\alpha / 6)$ as in Eq.(34). Lee, Low and Pines evaluated also the corrections due to off-diagonal parts of the transformed Hamiltonian and found that they are small.

\section{All-coupling continuum polaron}

\section{Feynman theory}

Feynman developed a superior all-coupling continuum-polaron theory using his pathintegral formalism (Feynman, 1955). He got the idea to formulate the polaron problem into the Lagrangian form of quantum mechanics and then eliminate the field oscillators, "... in exact analogy to Q. E. D.... (resulting in) ... a sum over all trajectories ...". The resulting path integral (here limited to the ground-state properties) is of the form:

$$
\langle 0, \beta \mid 0,0\rangle=\int \mathcal{D} \mathbf{r}(\tau) e^{S},
$$




$$
S=\exp \left[-\int_{0}^{\beta} \frac{\dot{\mathbf{r}}^{2}}{2} d \tau+\frac{\alpha}{2^{3 / 2}} \int_{0}^{\beta} \int_{0}^{\beta} \frac{e^{-|\tau-\sigma|}}{|\mathbf{r}(\tau)-\mathbf{r}(\sigma)|} d \tau d \sigma\right],
$$

where $\beta=1 / T$. Here, the Feynman units are used: $\hbar=1, m=1, \omega_{0}=1$. Eq. (48) gives the amplitude that an electron found at a point in space at time zero will appear at the same point at the imaginary time $\beta$. The interaction term in the action function $S$ may be interpreted as indicating that at "time" $\tau$, the electron behaves as if it were in a potential

$$
\frac{\alpha}{2^{3 / 2}} \int_{0}^{\beta} \frac{e^{-|\tau-\sigma|}}{|\mathbf{r}(\tau)-\mathbf{r}(\sigma)|} d \sigma
$$

which results from the electrostatic interaction of the electron with the mean charge density of its "previous" positions, weighted with the function $e^{-|\tau-\sigma|}$. This path integral (48) with (49) has a great intuitive appeal: it shows the polaron problem as an equivalent oneparticle problem in which the interaction, non-local in time or "retarded", occurs between the electron and itself.

Subsequently Feynman introduced a variational principle for path integrals to study the polaron. He then simulated the interaction between the electron and the polarization modes by a harmonic interaction (with force constant $k$ ) between a hypothetical ("fictitious") particle with mass $M$ and the electron. Within his model, the action function $S$ (49) is imitated by a quadratic trial action (non-local in time):

$$
S_{0}=\exp \left[-\int_{0}^{\beta} \frac{\dot{\mathbf{r}}^{2}}{2} d \tau+\frac{C}{2} \int_{0}^{\beta} \int_{0}^{\beta}[\mathbf{r}(\tau)-\mathbf{r}(\sigma)]^{2} e^{-w|\tau-\sigma|} d \tau d \sigma\right],
$$

where the interaction potential (50) is replaced by a parabolic potential

$$
\frac{C}{2} \int_{0}^{\beta}[\mathbf{r}(\tau)-\mathbf{r}(\sigma)]^{2} e^{-w|\tau-\sigma|} d \sigma
$$

with the weight function $e^{-w|\tau-\sigma|}$. The variational parameters $C$ and $w$ in Eq. (52) are adjusted in order to partly compensate for the error of exploiting the trial potential (52) instead of the true potential (50). Following the Feynman approach, an upper bound for the polaron ground-state energy can be written down as

$$
E=E_{0}-\lim _{\beta \rightarrow \infty} \frac{1}{\beta}\left\langle S-S_{0}\right\rangle_{0},
$$

where $S$ is the exact action functional of the polaron problem, while $S_{0}$ is the trial action functional, which corresponds to the above model system, $E_{0}$ is the ground-state energy of the model system, and

$$
\langle F\rangle_{0} \equiv \frac{\int F e^{S_{0}} \mathcal{D} \mathbf{r}(t)}{\int e^{S_{0}} \mathcal{D} \mathbf{r}(t)}
$$


The parameters of the model system $C$ and $w$ are found from the variational condition that they provide a mimimum to the upper bound for the ground state energy of Eq. (53)). (For the details of the calculation, see subsection [II.J) At nonzero temperatures, the best values of the model parameters can be determined from a variational principle for the free energy (Feynman, 1972), see Refs. (Krivoglaz and Pekar, 1957; Osaka, 1959).

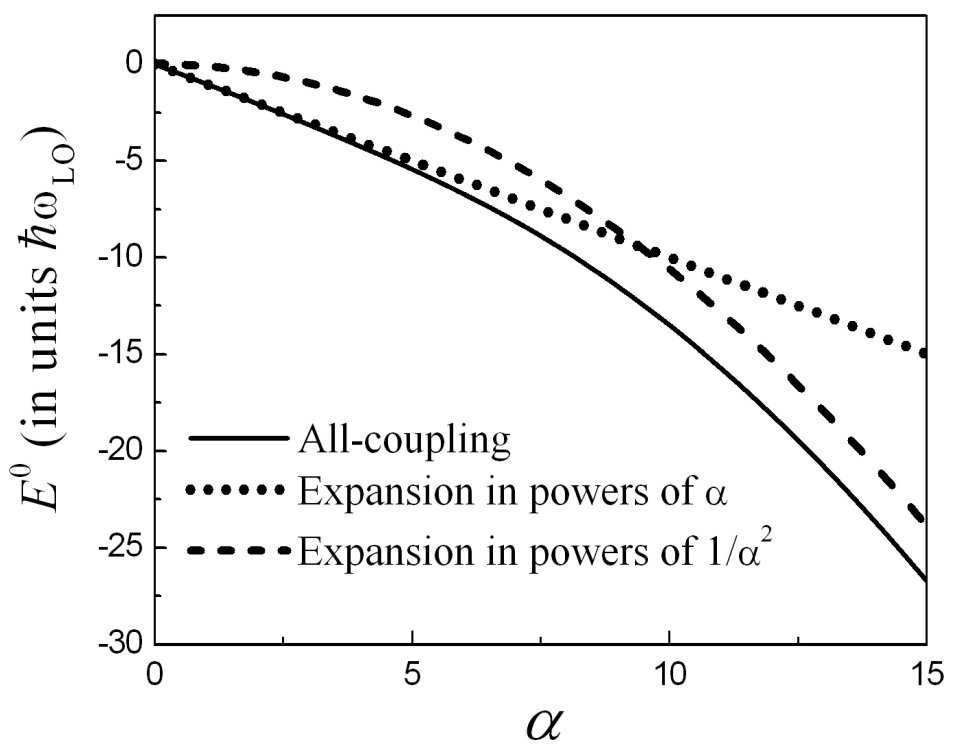

FIG. 1 Feynman-polaron energy as a function of $\alpha$ : the all-coupling theory.

Eq.(53) constitutes an upper bound for the polaron self-energy at all $\alpha$, which in Fig. 1 is compared with the results of weak- and strong-coupling expansions. The weak-coupling expansions of Feynman for the ground-state energy and the effective mass of the polaron are:

$$
\begin{gathered}
\frac{E_{0}}{\omega_{0}}=-\alpha-0.0123 \alpha^{2}-0.00064 \alpha^{3}-\ldots(\alpha \rightarrow 0), \\
\frac{m^{*}}{m}=1+\frac{\alpha}{6}+0.025 \alpha^{2}+\ldots(\alpha \rightarrow 0) .
\end{gathered}
$$

In the strong-coupling limit Feynman found for the ground-state energy:

$$
\frac{E_{0}}{\omega_{0}} \equiv \frac{E_{3 D}(\alpha)}{\omega_{0}}=-0.106 \alpha^{2}-2.83-\ldots \quad(\alpha \rightarrow \infty)
$$

and for the polaron mass:

$$
\frac{m^{*}}{m} \equiv \frac{m_{3 D}^{*}(\alpha)}{m}=0.0202 \alpha^{4}+\ldots \quad(\alpha \rightarrow \infty) .
$$


Becker et al. (1983), using a Monte Carlo calculation, derived the ground-state energy of a polaron as $E_{0}=\lim _{\beta \rightarrow \infty} \Delta F$, where $\Delta F=F_{\beta}-F_{\beta}^{0}$ with $F_{\beta}$ the free energy per polaron and $F_{\beta}^{0}=[3 /(2 \beta)] \ln (2 \pi \beta)$ the free energy per electron. The value $\beta \omega_{0}=25$, used for the actual computation in Ref. (Becker et al. , 1983), corresponds to $T / T_{D}=0.04\left(T_{D}=\hbar \omega_{\mathrm{LO}} / k_{B}\right.$; $\omega_{\mathrm{LO}} \equiv \omega_{0}$ is the longitudinal (LO) optical phonon frequency in conventional units). The authors of Ref. (Becker et al., 1983) actually calculated the free energy $\Delta F$, rather than the polaron ground-state enerqy. To investigate the importance of temperature effects on $\Delta F$, (Peeters and Devreese), 1985) considered the polaron energy as obtained by Osaka (1959), who generalized the Feynman polaron theory to nonzero temperatures:

$$
\begin{aligned}
\frac{\Delta F}{\omega_{0}}=\frac{3}{\beta} \ln & \left(\frac{w}{v} \frac{\sinh \frac{\beta_{0} v}{2}}{\sinh \frac{\beta_{0} w}{2}}\right)-\frac{3}{4} \frac{v^{2}-w^{2}}{v}\left(\operatorname{coth} \frac{\beta_{0} v}{2}-\frac{2}{\beta_{0} v}\right) \\
& -\frac{\alpha}{\sqrt{2 \pi}}\left[1+n\left(\omega_{0}\right)\right] \int_{0}^{\beta_{0}} d u \frac{e^{-u}}{\sqrt{D(u)}},
\end{aligned}
$$

where $\beta_{0}=\beta \omega_{0}, n(\omega)=1 /\left(e^{\beta \omega}-1\right)$, and

$$
D(u)=\frac{w^{2}}{v^{2}} \frac{u}{2}\left(1-\frac{u}{\beta_{0}}\right)+\frac{v^{2}-w^{2}}{2 v^{3}}\left(1-e^{-v u}-4 n(v) \sinh ^{2} \frac{v u}{2}\right) .
$$

This result is variational, with variational parameters $v$ and $w$, and gives an upper bound to the exact polaron free energy. $-\Delta F$ increases with increasing temperature and the effect of temperature on $\Delta F$ increases with increasing $\alpha$. The values for the free energy obtained analytically from the Feynman polaron model are lower than the published MC results for $\alpha \lesssim 2$ and $\alpha \geq 4$ (but lie within the $1 \%$ error of the Monte Carlo results). Since the Feynman result for the polaron free energy is an upper bound to the exact result, we conclude that for $\alpha \lesssim 2$ and $\alpha \geq 4$ the results of the Feynman model are closer to the exact result than the $\mathrm{MC}$ results of (Becker et al., 1983).

\section{Diagrammatic Monte-Carlo algorithm}

Mishchenko et al. (2000) performed a study of the Fröhlich polaron on the basis of the Diagrammatic Quantum Monte Carlo (DQMC) method (Prokof'ev and Svistunov, 1998). This method is based on the direct summation of Feynman diagrams for Green's functions in momentum space. The basic object of their investigation is the Matsubara Green's function of the polaron in the momentum $(\mathbf{k})$-imaginary time $(\tau)$ representation

$$
G(\mathbf{k}, \tau)=\left\langle\operatorname{vac}\left|c_{\mathbf{k}}(\tau) c_{\mathbf{k}}^{\dagger}(0)\right| \operatorname{vac}\right\rangle, \tau \geq 0
$$


TABLE I Comparison between the free energy of the Feynman polaron theory, $-(\Delta F)_{\mathrm{F}}$, and the Monte Carlo results of Ref. (Becker et al., 1983), $-(\Delta F)_{\mathrm{MC}}$, for $T / T_{D}=0.04$. The relative difference is defined as $\Delta=100 \times\left[(\Delta F)_{\mathrm{F}}-(\Delta F)_{\mathrm{MC}}\right] /(\Delta F)_{\mathrm{MC}}$. (From (Peeters and Devreese), 1985))

\begin{tabular}{|c|c|c|c|}
\hline \hline$\alpha$ & $-(\Delta F)_{\mathrm{F}}$ & $-(\Delta F)_{\mathrm{MC}}$ & $\Delta(\%)$ \\
\hline 0.5 & 0.50860 & 0.505 & 0.71 \\
\hline 1.0 & 1.02429 & 1.020 & 0.42 \\
\hline 1.5 & 1.54776 & 1.545 & 0.18 \\
\hline 2.0 & 2.07979 & 2.080 & -0.010 \\
\hline 2.5 & 2.62137 & 2.627 & -0.21 \\
\hline 3.0 & 3.17365 & 3.184 & -0.32 \\
\hline 3.5 & 3.73814 & 3.747 & -0.24 \\
\hline 4.0 & 4.31670 & 4.314 & 0.063 \\
\hline \hline & \multicolumn{3}{l}{$\mid-H \tau)}$. \\
$c_{\mathbf{k}}(\tau)=\exp (H \tau) c_{\mathbf{k}} \exp (-H \tau)$
\end{tabular}

In terms of a complete set $\{|\nu\rangle\}$ of eigenstates of the Fröhlich polaron Hamiltonian $H$, so that for a given $\mathbf{k}$ the relation $H|\nu(\mathbf{k})\rangle=E_{\nu}(\mathbf{k}) \mid \nu((k)\rangle$ and $H \mid$ vac $\rangle=E_{0}|\mathrm{vac}\rangle$, the expansion of the Green's function (61)

$$
G(\mathbf{k}, \tau)=\sum_{\nu}\left|\left\langle\nu\left|c_{\mathbf{k}}^{\dagger}\right| \operatorname{vac}\right\rangle\right|^{2} \exp \left\{-\left[E_{\nu}(\mathbf{k})-E_{0}\right] \tau\right\}
$$

follows straightforwardly. For the calculations discussed in what follows, $E_{0}=0$.

The spectral function (Lehmann function) $g_{\mathbf{k}}(\omega)$ is defined through the representation of the Green's function (63) in the form

$$
\begin{aligned}
G(\mathbf{k}, \tau) & =\int_{0}^{\infty} d \Omega \quad g_{\mathbf{k}}(\Omega), \\
g_{\mathbf{k}}(\Omega) & =\sum_{\nu} \delta\left[\Omega-E_{\nu}(\mathbf{k})\right]\left|\left\langle\nu\left|c_{\mathbf{k}}^{\dagger}\right| \operatorname{vac}\right\rangle\right|^{2} .
\end{aligned}
$$

This spectral function is normalized, $\int_{0}^{\infty} g_{\mathbf{k}}(\Omega) d \Omega=1$. It can be interpreted as the probability that a polaron has momentum $\mathbf{k}$ and energy $\Omega$. The significance of the spectral function (65) is determined by the fact that it has poles (sharp peaks) at frequencies, which correspond to stable (metastable) quasiparticle states. 
If, for a given $\mathbf{k}$, there is a stable state at energy $E(\mathbf{k})$, the spectral function takes the form

$$
g_{\mathbf{k}}(\Omega)=Z_{0}^{(\mathbf{k})} \delta[\Omega-E(\mathbf{k})] \ldots,
$$

where $Z_{0}^{(\mathbf{k})}$ is the weight of the bare-electron state. The energy $E_{\mathrm{gs}}(\mathbf{k})$ and the weight $Z_{0, \mathrm{gs}}^{(\mathbf{k})}$ for the polaron ground state can be extracted from the Green's function behaviour at long times:

$$
G\left(\mathbf{k}, \tau \gg \omega_{0}^{-1}\right) \rightarrow Z_{0}^{(\mathbf{k})} \exp [-E(\mathbf{k}) \tau]
$$

Similarly to Eq. (61), the $N$-phonon Green's function is defined:

$$
\begin{aligned}
G_{N}\left(\mathbf{k}, \tau ; \mathbf{q}_{1}, \ldots, \mathbf{q}_{N}\right) & =\left\langle\operatorname{vac}\left|d_{\mathbf{q}_{N}}(\tau) \ldots d_{\mathbf{q}_{1}}(\tau) c_{\mathbf{p}}(\tau) c_{\mathbf{p}}^{\dagger}(0) d_{\mathbf{q}_{1}}^{\dagger}(0) \ldots d_{\mathbf{q}_{N}}^{\dagger}(0)\right| \operatorname{vac}\right\rangle, \tau \geq 0, \\
\mathbf{p} & =\mathbf{k}-\sum_{j-1}^{N} \mathbf{q}_{j} .
\end{aligned}
$$

From the asymptotic properties of the Green's functions (68) at long times, the characteristics of the polaron ground state are found. In particular, the weight of the $N$-phonon state for the polaron ground state is given by

$$
G_{N}\left(\mathbf{k}, \tau \gg \omega_{0}^{-1} ; \mathbf{q}_{1}, \ldots, \mathbf{q}_{N}\right) \rightarrow Z_{N}^{(\mathbf{k})}\left(\mathbf{q}_{1}, \ldots, \mathbf{q}_{N}\right) \exp [-E(\mathbf{k}) \tau]
$$

A standard diagrammatic expansion of the above described Green's functions generates a series of Feynman diagrams. The following function is further introduced:

$$
P(\mathbf{k}, \tau)=G(\mathbf{k}, \tau)+\sum_{N=1}^{\infty} \int d \mathbf{q}_{1} \ldots d \mathbf{q}_{N} \tilde{G}_{N}\left(\mathbf{k}, \tau ; \mathbf{q}_{1}, \ldots, \mathbf{q}_{N}\right),
$$

where $\tilde{G}_{N}$ are irreducible $N$-phonon Green's functions (which do not contain disconnected phonon propagators). From Eqs. (67), (69) and the completeness condition for the nondegenerate ground state

$$
Z_{0}^{(\mathbf{k})}+\sum_{N=1}^{\infty} \int d \mathbf{q}_{1} \ldots d \mathbf{q}_{N} Z_{N}^{(\mathbf{k})}\left(\mathbf{q}_{1}, \ldots, \mathbf{q}_{N}\right)=1
$$

it follows that the polaron ground state energy is determined by the asymptotic behaviour of the function (70):

$$
P\left(\mathbf{k}, \tau \gg \omega_{0}^{-1}\right) \rightarrow \exp [-E(\mathbf{k}) \tau]
$$

The function $P(\mathbf{k}, \tau)$ is an infinite series of integrals containing an ever increasing number of integration variables. The essence of the DQMC method is to construct a process, which 
generates continuum random variables $(\mathbf{k}, \tau)$ with a distribution function that coincides exactly with $P(\mathbf{k}, \tau)$. Taking into account Eq. (70) and the diagrammatic rules, $P(\mathbf{k}, \tau)$ is identified with the distribution function

$$
Q(\{y\})=\sum_{m=0}^{\infty} \sum_{\xi_{m}} \int d x_{1} \ldots d x_{m} F_{m}\left(\xi_{m},\{y\}, x_{1}, \ldots, x_{m}\right),
$$

where the external variables $\{y\}$ include $\mathbf{k}, \tau, \alpha$ and $N$, while the internal variables describe the topology of the diagram (labelled with $\xi_{m}$ ), times assigned to electron-phonon vertices and momenta of phonon propagators. The diagrammatic Monte Carlo process is a numeric procedure, which samples various diagrams in parameter space and collects statistics for $Q(\{y\})$ according to the Metropolis algorithm (Metropolis et al., 1953) is such a way that — when the process is repeated a large number of times — the result converges to the exact answer. The distribution function given by the convergent series (73) is simulated within the process of sequential stochastic generation of diagrams described by functions $F_{m}\left(\xi_{m},\{y\}, x_{1}, \ldots, x_{m}\right)$. Further, using Eq. (64), the spectral function $g_{\mathbf{k}}(\omega)$ is obtained applying a stochastic optimization technique. We refer to the review (Mishchenko and Nagaosa, 2007) for further details on the DQMC and stochastic optimization, where information on the excited states of the polaron is also derived by the analytic continuation of the imaginary time Green's functions to real frequencies.

DQMC confirms that for $\alpha \gtrsim 1$ the bare-electron state in the polaron wave function is no longer the dominant contribution and perturbation theory is not adequate. The bareelectron weight $Z_{0}^{0}$ for the polaron ground state, as a function of the polaron coupling constant, rapidly vanishes for $\alpha \gtrsim 3$. It is suggested in (Mishchenko et al., 2000) that in the interval $3 \lesssim \alpha \lesssim 10$ the polaron ground states smoothly transforms between weak- and strong-coupling limits.

Below (see sections III.C, III.D, III.G) earlier analytical studies and results on Fröhlich polarons are compared with DQMC results. It would be beneficial, to have an independent numerical check of the DQMC results. The comparison of the DQMC results for the "low-energy" $(\Omega<0)$ part of the spectral density ${ }^{1}$ for the Fröhlich polaron at $\alpha=0.05$ (Mishchenko et al., 2000) demonstrates perfect agreement with the perturbation theory re-

\footnotetext{
${ }^{1}$ It is worth of noticing, that the spectral density is not identical to the optical absorption coefficient, which is discussed below in sections ПII.B, ПII.C and III.D
} 
sult:

$$
g_{0}(\Omega<0)=\frac{\alpha}{2} \delta[\Omega+\alpha]
$$

( $m$ and $\omega_{0}$ are set equal to unity). The DQMC results for the "high-energy" $(\Omega>0)$ part of the spectral density significantly differ from the perturbation-theory curve. This is attributed to the fact that for the Fröhlich polaron the perturbation-theory expression for

$$
g_{0}(\Omega>0)=\frac{\alpha}{\pi} \frac{\theta(\Omega-1)}{\Omega^{2} \sqrt{\Omega-1}}
$$

diverges as $\Omega \rightarrow 1$ and, consequently, the perturbation approach is no longer adequate. The main difference between the DQMC spectrum of the Fröhlich polaron and the perturbationtheory result is the broad peak in the spectral density at $\Omega \sim 3.5$. This peak appears for significantly larger values of the coupling constant and its weight grows with $\alpha$, see Fig. 2 , As shown in the inset, near the threshold, $\Omega=1$, the spectral density behaves as $\sqrt{\Omega-1}$.

Next to Fröhlich polarons, other polaron models have been extensively investigated using the Monte Carlo approach. In particular Alexandrov (1996) proposed a long-range discrete Fröhlich interaction as a model of the interaction between a hole and the oxygen ions in cuprate superconductors. The essence of the model is that a charge carrier moves from site to site on a discrete lattice (or chain in 1D) and interacts with all the ions, which reside at the lattice sites. Numerically rigorous polaron characteristics (ground-state energy, number of phonons in the polaron cloud, effective mass and isotope exponent) for such a lattice polaron, valid for arbitrary EPI, were obtained using a path-integral continuous-time quantum Monte Carlo technique (CTQMC) (Alexandrov and Kornilovitch, 1999; Hague et al. 2006a; Spencer et al. , 2005). This study leads to a "mobile small Fröhlich" polaron (IV.E.3).

Over the years the Feynman model for the Fröhlich polaron has remained the most successful approach to the problem. The analysis of an exactly solvable ("symmetrical") 1Dpolaron model (Devreese,, 1964; Devreese and Evrard, 1964, 1968), Monte Carlo schemes (Mishchenko et al., 2000; Titantah et al., 2001) and, recently, a unifying variational approach (Cataudella et al., 2007; De Filippis et al. , 2003) demonstrate the remarkable accuracy of Feynman's path-integral method. Using the variational wave-functions, which combine both the Landau-Pekar and the Feynman approximations, (Cataudella et al., 2007) found in the $\alpha \rightarrow \infty$ limit:

$$
E_{0}=\left(-0.108507 \alpha^{2}-2.67\right) \omega_{0}
$$




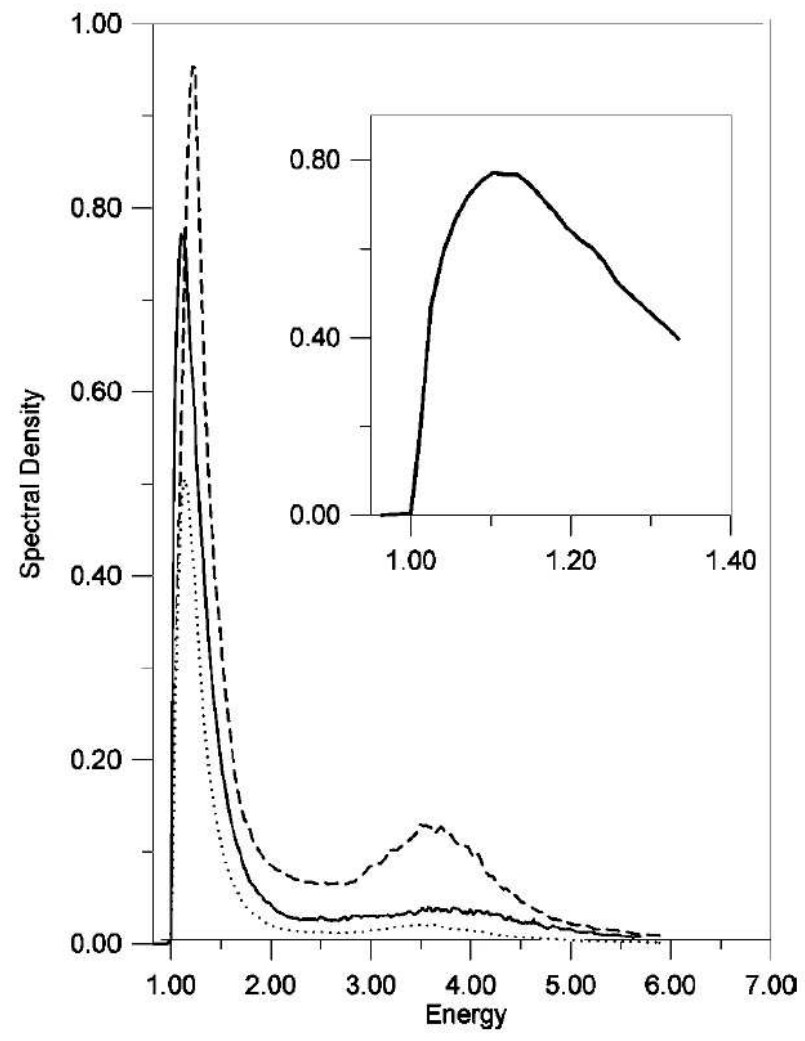

FIG. 2 The spectral density of the Fröhlich polaron for various values of the coupling constant: $\alpha=0.5$ (dotted line), $\alpha=1$ (solid line), and $\alpha=2$ (dashed line) with energy counted from the ground-state energy of the polaron. The initial fragment of the spectral density for $\alpha=1$ is shown in the inset (from (Mishchenko et al., 2000)).

which is slightly lower than the variational Feynman's estimate, Eq. (50), and at small values of $\alpha$,

$$
E_{0}=-\alpha \omega_{0}-0.0123 \alpha^{2} \omega_{0}, \quad \alpha \rightarrow 0
$$

i.e. the same result, as Eq. (55). In the latter limit the correct result for the electron self-energy is

$$
E=-\alpha \omega_{0}-0.0159 \alpha^{2} \omega_{0}
$$

as found by Grosjean (1957) and confirmed by Höhler and Mullensiefen (1959), and Röseler (1968).

Within the path-integral approach, Feynman et al. studied the mobility of polarons (Feynman et al., 1962; Thornber and Feynman, 1970). Subsequently the path-integral approach to the polaron problem was generalized and developed to become a power- 
ful tool to study optical absorption and cyclotron resonance (Devreese et al., 1972a; Peeters and Devreese, 1986).

\section{RESPONSE OF CONTINUUM POLARONS}

\section{A. Mobility}

The mobility of large polarons was studied within various theoretical approaches. Fröhlich (Fröhlich , 1937, 1954) pointed out the typical behavior of the large-polaron mobility

$$
\mu \propto \exp \left(\omega_{0} \beta\right)
$$

which is characteristic for weak coupling. Within the weak-coupling regime, the mobility of the polaron was then derived, e. g., using the Boltzmann equation in (Howarth and Sondheimer, 1953; Osaka , 1961) and starting from the LLP-transformation in Ref. (Low and Pines, 1955). In (Langreth and Kadanoff, 1964) it was shown that for weak coupling:

$$
\mu=\frac{e}{2 \alpha}\left[\exp \left(\omega_{0} \beta\right)-1\right]\left[1-\frac{\alpha}{6}+O\left(\alpha^{2}\right)\right] .
$$

A nonperturbative analysis was based on the Feynman polaron theory, where the mobility $\mu$ of the polaron using the path-integral formalism was derived by Feynman et al. (1962) (FHIP) as a static limit, starting from a frequency-dependent impedance function. Details of the FHIP theory are given in (Platzman, 1963). An approximate expression for the impedance function of a Fröhlich polaron at all frequencies, temperatures and coupling strengths was obtained in Refs. (Feynman et al., 1962; Platzman, 1963) within the pathintegral technique. Assuming the crystal to be isotropic, an alternating electric field $\mathbf{E}=$ $E_{0} \mathbf{e}_{x} \exp (i \Omega t)$ induces a current in the $x$-direction

$$
j(\Omega)=[z(\Omega)]^{-1} E_{0} \exp (i \Omega t) .
$$

The complex function $z(\Omega)$ is called the impedance function. The electric field is considered sufficiently weak, so that linear-response theory can be applied. The frequency-depending mobility $\mu(\Omega)$ is defined as

$$
\mu(\Omega)=\operatorname{Re}[z(\Omega)]^{-1}
$$


Taking the electric charge as unity, one arrives at $j=\langle\dot{x}\rangle$, where $\langle x\rangle$ is the expectation value of the electron displacement in the direction of the applied field: $\langle x\rangle=E / i \Omega z(\Omega)$. In terms of time dependent variables,

$$
\langle x(\tau)\rangle=-\int_{-\infty}^{\tau} i G(\tau-\sigma) E(\sigma) d \sigma,
$$

where $G(\tau)$ has the inverse Fourier transform

$$
G(\Omega)=\int_{-\infty}^{\infty} G(\tau) \exp (-i \Omega \tau) d \tau=[\Omega z(\Omega)]^{-1} .
$$

The expected displacement at time $\tau$ is

$$
\langle x(\tau)\rangle=\operatorname{Tr}\left[x U(\tau, a) \rho_{a} U^{\prime-1}(\tau, a)\right] .
$$

Here, $\rho_{a}$ is the density matrix of the system at some time $a$, long before the field is turned on, and

$$
U(\tau, a)=\mathrm{T} \exp \left\{-i \int_{a}^{\tau}\left[H_{s}-x_{s} E(s)\right] d s\right\}
$$

is the unitary operator of the development of a state in time with the complete Hamiltonian $H-x E$, where $H$ is the Fröhlich polaron Hamiltonian, and $\mathrm{T}$ is the time ordering operator (Feynman, 1951). Primed operators are ordered antichronologically:

$$
U^{\prime-1}(\tau, a)=\mathrm{T}^{\prime} \exp \left\{i \int_{a}^{\tau}\left[H_{s}^{\prime}-x_{s}^{\prime} E^{\prime}(s)\right] d s\right\} .
$$

$G(\tau-\sigma)$ can be represented as the second derivative

$$
G(\tau-\sigma)=\left.\frac{1}{2}\left(\frac{\partial^{2} g}{\partial \eta \partial \varepsilon}\right)\right|_{\varepsilon=\eta=0}
$$

of

$$
g=\operatorname{Tr}\left[U(b, a) \rho_{a} U^{\prime-1}(b, a)\right], \quad b \rightarrow \infty, \quad a \rightarrow-\infty
$$

with $E(s)=\varepsilon \delta(s-\sigma)+\eta \delta(s-\tau), E^{\prime}(s)=\varepsilon \delta(s-\sigma)-\eta \delta(s-\tau)$. The initial state is chosen at a definite temperature $T, \rho_{a} \propto \exp (-\beta H)$. If the time $a$ is sufficiently far in the past, FHIP assume that only the phonon subsystem was in thermal equilibrium at temperature $T$. The energy of the single electron and of the electron-phonon coupling are infinitesimal (of order 
$1 / V)$ with respect to that of the phonons. With this choice of the initial distribution, the phonon coordinates can be eliminated from the expression (89), and the entire expression is reduced to a double path integral over the electron coordinates only:

$$
g=\iint \exp (i \Phi) \mathcal{D} \mathbf{r}(t) \mathcal{D} \mathbf{r}^{\prime}(t)
$$

where (taking $m=1$ )

$$
\begin{aligned}
\Phi= & \int_{-\infty}^{\infty}\left[\frac{\dot{\mathbf{r}}^{2}}{2}-\frac{\dot{\mathbf{r}}^{\prime 2}}{2}\right] d t-\int_{-\infty}^{\infty}\left[\mathbf{E}(t) \cdot \mathbf{r}(t)-\mathbf{E}^{\prime}(t) \cdot \mathbf{r}^{\prime}(t)\right] d t \\
& +\frac{i \alpha}{2^{3 / 2}} \int_{-\infty}^{\infty} \int_{-\infty}^{\infty}\left[\frac{\exp (-i|t-s|)+2 P(\beta) \cos (t-s)}{|\mathbf{r}(t)-\mathbf{r}(s)|}+\frac{\exp (+i|t-s|)+2 P(\beta) \cos (t-s)}{\left|\mathbf{r}^{\prime}(t)-\mathbf{r}^{\prime}(s)\right|}\right. \\
& \left.-\frac{2[\exp (-i(t-s))+2 P(\beta) \cos (t-s)]}{\left|\mathbf{r}^{\prime}(t)-\mathbf{r}(s)\right|}\right] d t d s,
\end{aligned}
$$

where $P(\beta)=\left[e^{\beta}-1\right]^{-1}$. The double path integral in (90) is over paths which satisfy the boundary condition $\mathbf{r}(t)-\mathbf{r}^{\prime}(t)=0$ at times $t$ approaching $\pm \infty$. The expression (90) with (91) is supposed to be exact (Feynman et al., 1962). Clearly to provide analytical solutions at all $\alpha$ presumably is impossible.

Following Feynman's idea to describe the ground state energy properties of a polaron by introducing a parabolic "retarded" interaction of the electron with itself (see subsection II.D), it is assumed in (Feynman et al., 1962) that the dynamical behavior of the polaron can be described approximately by replacing $\Phi$ of Eq. (90) by a parabolic (retarded) expression

$$
\begin{aligned}
\Phi_{0}= & \int_{-\infty}^{\infty}\left[\frac{\dot{\mathbf{r}}^{2}}{2}-\frac{\dot{\mathbf{r}}^{\prime 2}}{2}\right] d t-\int_{-\infty}^{\infty}\left[\mathbf{E}(t) \cdot \mathbf{r}(t)-\mathbf{E}^{\prime}(t) \cdot \mathbf{r}^{\prime}(t)\right] d t \\
& -\frac{i C}{2} \int_{-\infty}^{\infty} \int_{-\infty}^{\infty}\left\{[\mathbf{r}(t)-\mathbf{r}(s)]^{2}\left[e^{-i w|t-s|}+2 P(\beta w) \cos w(t-s)\right]\right. \\
& +\left[\mathbf{r}^{\prime}(t)-\mathbf{r}^{\prime}(s)\right]^{2}\left[e^{+i w|t-s|}+2 P(\beta w) \cos (t-s)\right] \\
& \left.-2\left[\mathbf{r}^{\prime}(t)-\mathbf{r}(s)\right]^{2}\left[e^{-i w(t-s)}+2 P(\beta w) \cos w(t-s)\right]\right\} d t d s
\end{aligned}
$$

The parameters $C$ and $w$ are to be determined so as to approximate $\Phi$ as closely as possible. However, no variational principle is known for the mobility. At zero temperature, $C$ and $w$ are fixed at the values, which provide the best upper bound for the polaron ground state 
energy (53). At finite temperatures $(T \neq 0)$ the parameters $C$ and $w$ are determined from the variational principle for the polaron free energy (Feynman, 1972). This way of selection of the model parameters $C$ and $w$ is based on the supposition, that "the comparison Lagrangian, which gives a good fit to the ground-state energy at zero temperature, will also give the dynamical behaviour of the system" (Feynman et al., 1962).

The analytical calculation of path integrals in (90) in (Feynman et al., 1962) was performed to the first term in an expansion of $\exp \left[i\left(\Phi-\Phi_{0}\right)\right]$ :

$$
\begin{aligned}
g & =\iint \exp \left(i \Phi_{0}\right) \exp \left[i\left(\Phi-\Phi_{0}\right)\right] \mathcal{D} \mathbf{r}(t) \mathcal{D} \mathbf{r}^{\prime}(t) \approx g_{0}+g_{1}, \\
g_{0} & =\iint \exp \left(i \Phi_{0}\right) \mathcal{D} \mathbf{r}(t) \mathcal{D} \mathbf{r}^{\prime}(t), \\
g_{1} & =i \iint \exp \left(i \Phi_{0}\right)\left(\Phi-\Phi_{0}\right) \mathcal{D} \mathbf{r}(t) \mathcal{D} \mathbf{r}^{\prime}(t) .
\end{aligned}
$$

Using (84) and (88), one finds from(93)

$$
G(\Omega) \approx G_{0}(\Omega)+G_{1}(\Omega)
$$

The first term in the rhs of Eq. (96) is

$$
G_{0}(\Omega)=i Y_{0}(\Omega), \quad Y_{0}(\Omega)=-\frac{\Omega^{2}-w^{2}}{(\Omega-i \varepsilon)^{2}\left[(\Omega-i \varepsilon)^{2}-v^{2}\right]}, \quad \varepsilon \rightarrow+0
$$

with $v^{2}=w^{2}+4 C / w$. The second term in the rhs of Eq. (96) is

$$
\begin{aligned}
G_{1}(\Omega)= & -i Y_{0}^{2}(\Omega)\left[\chi(\Omega)-\frac{4 C}{w} \frac{\Omega^{2}}{\Omega^{2}-w^{2}}\right] \\
\chi(\Omega)= & \int_{0}^{\infty}\left(1-e^{i \Omega u}\right) \operatorname{Im} S(u) d u \\
S(u)= & \frac{2 \alpha}{3 \sqrt{\pi}} \frac{e^{i u}+2 P(\beta) \cos u}{[D(u)]^{3 / 2}} \\
D(u)= & \frac{w^{2}}{v^{2}}\left\{\frac{v^{2}-w^{2}}{w^{2} v}\left[1-e^{i v u}+4 P(\beta v) \sin ^{2}\left(\frac{v u}{2}\right)\right]\right. \\
& \left.-i u+\frac{u^{2}}{\beta}\right\} .
\end{aligned}
$$

From (84) and (96), the impedance results in the form

$$
\Omega z(\Omega) \approx\left[G_{0}(\Omega)+G_{1}(\Omega)\right]^{-1} .
$$

Feynman et al.(1962) suggested to use this expression expanded to first order in $G_{1}(\Omega)$ :

$$
\Omega z(\Omega) \approx\left[G_{0}(\Omega)\right]^{-1}-\left[G_{0}(\Omega)\right]^{-2} G_{1}(\Omega)
$$


Substitution of (97) and (98) into Eq.(103) leads to the final expression (Ref. (Feynman et al., 1962)) for the impedance function of the Fröhlich polaron:

$$
\Omega z(\Omega)=i\left[\Omega^{2}-\chi(\Omega)\right]
$$

An alternative derivation of the impedance function of the Fröhlich polaron, based on simple operator techniques, was presented in (Peeters and Devreese, 1983a). The FHIP result was worked out in (Peeters and Devreese, 1984) in detail to get a physical insight into the scattering processes incorporated in the FHIP approximation. For sufficiently low temperature the FHIP polaron mobility takes the form (Feynman et al., 1962)

$$
\mu=\left(\frac{w}{v}\right)^{3} \frac{3 e}{4 m \omega_{0}^{2} \alpha \beta} \mathrm{e}^{\omega_{0} \beta} \exp \left\{\left(v^{2}-w^{2}\right) / w^{2} v\right\}
$$

where $v$ and $w$ are variational functions of $\alpha$ obtained from the Feynman polaron model.

Using the Boltzmann equation for the Feynman polaron model, Kadanoff (1963) found the mobility, which for low temperatures can be represented as follows:

$$
\mu=\left(\frac{w}{v}\right)^{3} \frac{e}{2 m \omega_{0} \alpha} \mathrm{e}^{\omega_{0} \beta} \exp \left\{\left(v^{2}-w^{2}\right) / w^{2} v\right\}
$$

The weak-coupling perturbation expansion of the low-temperature polaron mobility as found using the Green's function technique (Langreth and Kadanoff), 1964) has confirmed that the mobility derived from the Boltzmann equation is asymptotically exact for weak coupling $(\alpha \ll 1)$ and at low temperatures $\left(T \ll \omega_{0}\right)$. As first noticed in (Kadanoff), 1963), the mobility of Eq. (105) differs by the factor of $3 /\left(2 \beta \omega_{0}\right)$ from that derived using the polaron Boltzmann equation as given by Eq. (106) ${ }^{2}{ }^{2}$ As follows from this comparison, the result of (Feynman et al., 1962) is not valid when $T \rightarrow 0$. As emphasized in (Fevnman et al., 1962) and later confirmed, in particular, in (Peeters and Devreese, 1983b, 1984) the above discrepancy can be attributed to an interchange of two limits in calculating the impedance. In FHIP, for weak electron-phonon coupling, one takes $\lim _{\Omega \rightarrow 0} \lim _{\alpha \rightarrow 0}$, whereas $\lim _{\alpha \rightarrow 0} \lim _{\Omega \rightarrow 0}$ should be calculated. It turns out that for the asymptotically correct result the mobility

\footnotetext{
${ }^{2}$ In the asymptotic limit of weak electron-phonon coupling and low temperature, the FHIP polaron mobility of Eq. (105) differs by the same factor of $3 /\left(2 \beta \omega_{0}\right)$ from the earlier result (Howarth and Sondheimer, 1953; Low and Pines, 1955; Osaka, 1961), which, as pointed out in Ref. (Fevnman et al., 1962) and in later publications (Kadanoff, 1963; Langreth and Kadanoff, 1964; Peeters and Devreese, 1984), is correct for $\beta \gg 1$.
} 
at low temperatures is mainly limited by the absorption of phonons, while in the theory of FHIP it is the emission of phonons which gives the dominant contribution as $T$ goes to zero (Peeters and Devreese, 1983b).

The analysis based on the Boltzmann equation takes into account the phonon emission processes whenever the energy of the polaron is above the emission threshold. The independent-collision model, which underlies the Boltzmann-equation approach, however, fails in the "strong coupling regime" of the Fröhlich polaron, when the thermal mean free path becomes less than the de Broglie wavelength; in this case, the Boltzmann equation cannot be expected to be adequate (Feynman et al., 1962; Hellwarth and Biaggio, 1999).

Experimental work on alkali halides and silver halides indicates that the mobility obtained from Eq. (105) describes the experimental results accurately (see (Brown, 1963, 1972) and references therein). Measurements of mobility as a function of temperature for photoexcited electrons in cubic $n$-type $\mathrm{Bi}_{12} \mathrm{SiO}_{20}$ are explained well in terms of large polarons within the Feynman approach (Hellwarth and Biaggio, 1999). The experimental findings on electron transport in crystalline $\mathrm{TiO}_{2}$ (rutile phase) probed by $\mathrm{THz}$ time-domain spectroscopy were quantitatively interpreted within the Feynman model (Hendry et al., 2004). One of the reasons for the agreement between theory based on Eq. (105) and experiment is that in the path-integral approximation to the polaron mobility, a Maxwellian distribution for the electron velocities is assumed, when applying the adiabatic switching on of the Fröhlich interaction. Although such a distribution is not inherent in the Fröhlich interaction, its incorporation tends to favor agreement with experiment because other mechanisms (interaction with acoustic phonons etc.) cause a Gaussian distribution.

\section{B. Optical absorption at weak coupling}

At zero temperature and in the weak-coupling limit, the optical absorption of a Fröhlich polaron is due to the elementary polaron scattering process with the absorption of incoming photon and emission of an outgoing phonon. In the weak-coupling limit $(\alpha \ll 1)$ the polaron absorption coefficient for a many-polaron gas was first obtained by Gurevich et al. (1962). Their optical-absorption coefficient is equivalent to a particular case of (Tempere and Devreese), 2001) with the dynamic structure factor $S(\mathbf{q}, \omega)$ corresponding to the Hartree-Fock approximation. In (Tempere and Devreese, 2001) the optical absorption 
coefficient of a many-polaron gas was shown to be given, to order $\alpha$, by

$$
\operatorname{Re}[\sigma(\Omega)]=n_{0} e^{2} \frac{2}{3} \alpha \frac{1}{2 \pi \Omega^{3}} \int_{0}^{\infty} d q q^{2} S\left(\mathbf{q}, \Omega-\omega_{0}\right),
$$

where $n_{0}$ is the density of charge carriers.

In the zero-temperature limit, starting from the Kubo formula the optical conductivity of a single Fröhlich polaron can be represented in the form

$\sigma(\Omega)=i \frac{e^{2}}{m(\Omega+i \delta)}+\frac{e^{2}}{m^{2}} \frac{1}{(\Omega+i \delta)^{3}} \int_{0}^{\infty} e^{-\delta t}\left(e^{i \Omega t}-1\right) \sum_{\mathbf{q}, \mathbf{q}^{\prime}} q_{x} q_{x}^{\prime}\left\langle\Psi_{0}\left|\left[\hat{B}_{\mathbf{q}}(t), \hat{B}_{-\mathbf{q}^{\prime}}(0)\right]\right| \Psi_{0}\right\rangle d t$

where $\delta=+0, \hat{B}_{\mathbf{q}}(t)=\left[V_{\mathbf{q}} d_{\mathbf{q}}(t)+V_{-\mathbf{q}}^{*} d_{-\mathbf{q}}^{\dagger}(t)\right] e^{i \mathbf{q} \cdot \mathbf{r}(t)}$, and $\left|\Psi_{0}\right\rangle$ is the ground-state wave function of the electron-phonon system. Within the weak coupling approximation, the following analytic expression for the real part of the polaron optical conductivity results from Eq. (108):

$$
\operatorname{Re} \sigma(\Omega)=\frac{\pi e^{2}}{2 m^{*}} \delta(\Omega)+\frac{2 e^{2}}{3 m} \frac{\omega_{0} \alpha}{\Omega^{3}} \sqrt{\Omega-\omega_{0}} \Theta\left(\Omega-\omega_{0}\right),
$$

where $\Theta(x)=1$ if $x>0$, and zero otherwise. The spectrum of the real part of the polaron optical conductivity (109) is represented in Fig. 3.

According to Eq. (109), the absorption coefficient of light with frequency $\Omega>0$ by free polarons for $\alpha \rightarrow 0$ takes the form

$$
\Gamma(\Omega)=\frac{4 \pi}{n c} \frac{2 n_{p} e^{2} \alpha \omega_{0}^{2}}{3 m \Omega^{3}}\left(\Omega / \omega_{0}-1\right)^{1 / 2} \quad \Theta\left(\Omega-\omega_{0}\right)
$$

where $c$ is the velocity of light in vacuum, $n$ is the refractive index of the medium, $n_{p}$ is the concentration of polarons. A simple derivation (Devreese et al. , 1971) using a canonical transformation method gives the absorption coefficient of free polarons, which coincides with the result (110). The step function in (110) reflects the fact that at zero temperature the absorption of light accompanied by the emission of a phonon can occur only if the energy of the incident photon is larger than that of a phonon $\left(\Omega>\omega_{0}\right)$. In the weakcoupling limit, according to Eq. (110), the absorption spectrum consists of a "one-phonon line". At nonzero temperature, the absorption of a photon can be accompanied not only by emission, but also by absorption of one or more phonons. Similarity between the temperature dependence of several features of the experimental infrared absorption spectra in high- $\mathrm{T}_{c}$ superconductors and the temperature dependence predicted for the optical absorption of a 


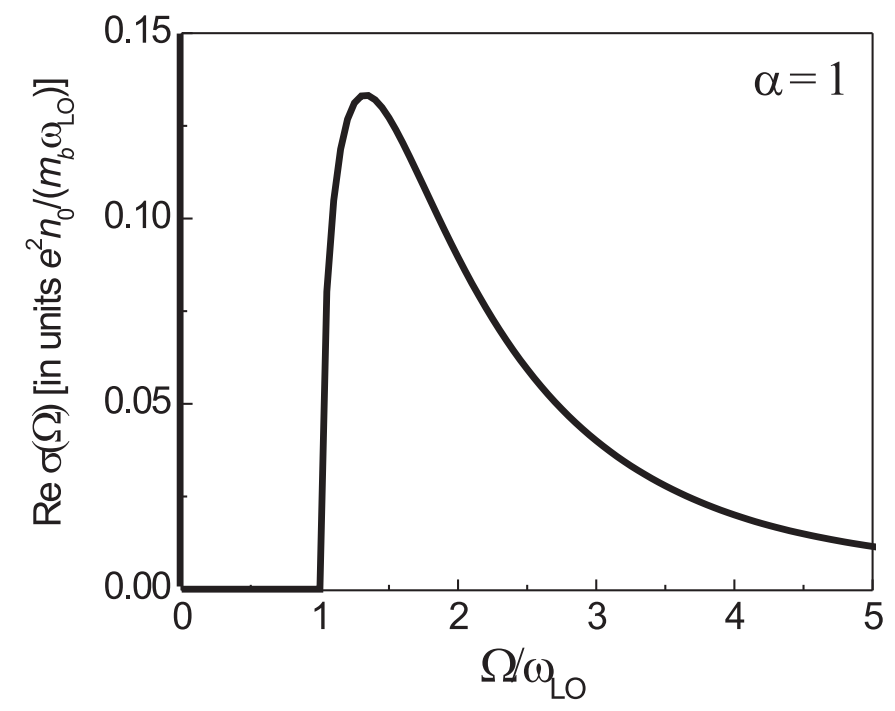

FIG. 3 Polaron optical conductivity for $\alpha=1$ in the weak-coupling approximation, according to (Devreese, 1972) A $\delta$-like central peak (at $\Omega=0$ ) is schematically shown by a vertical line. (Reprinted with permission from (Devreese, 2006a). (C)2006, Società Italiana di Fisica.)

single Fröhlich polaron (Devreese, 1972; Devreese et al. , 1972a) has been revealed in Ref. (Devreese and Tempere, 1998).

Experimentally, this one-phonon line has been observed for free polarons in the infrared absorption spectra of CdO-films (Finkenrath et al., 1969), which is a weakly polar material with $\alpha \approx 0.74$. The polaron absorption band is observed in the spectral region between 6 and $20 \mu \mathrm{m}$ (above the LO phonon frequency). The difference between the one-polaron theoretical absorption and experiment in the wavelength region where polaron absorption dominates the spectrum has been explained as due to many-polaron effects (Tempere and Devreese, 2001).

\section{Optical absorption at strong coupling}

The structure of the large polaron excitation spectrum constituted a central question at the early stages of the development of polaron theory. The exactly solvable polaron model of Ref. (Devreese, 1964) was used to demonstrate the existence of the so-called "relaxed excited states" of large polarons (Devreese and Evrard, 1964). In Ref. (Devreese, 1964), 
an exactly solvable ("symmetric") 1D-polaron model was introduced and analysed. The further study of this model was performed in Refs. (Devreese and Evrard, 1964, 1968). The model consists of an electron interacting with two oscillators possessing opposite wave vectors: $\mathbf{q}$ and $-\mathbf{q}$. The parity operator, which changes $d_{\mathbf{q}}$ and $d_{-\mathbf{q}}$ (and also $d_{\mathbf{q}}^{\dagger}$ and $d_{-\mathbf{q}}^{\dagger}$ ), commutes with the Hamiltonian of the system. Hence, the polaron states are classified into even and odd states with eigenvalues of the parity operator +1 and -1 , respectively. For the lowest even and odd states, the phonon distribution functions $W_{N}$ are plotted in Fig. 4, upper panel, for some values of the effective coupling constant $\lambda$ of this "symmetric" model. The value of the parameter $\varkappa=\left[q^{2} / m \omega_{0}\right]^{1 / 2}$ for these graphs was taken to be 1 , while the total polaron momentum $\mathbf{P}=0$. In the weak-coupling case $(\lambda \approx 0.6) W_{N}$ is a decaying function of $N$. When increasing $\lambda, W_{N}$ acquires a maximum, e. g. at $N=8$ for the lowest even state at $\lambda=5.0625$. The phonon distribution function $W_{N}$ has the same character for the lowest even and the lowest odd states at all values of the number of virtual phonons in the ground state (as distinct from the higher states). This led to the conclusion that the lowest odd state is an internal excited state of the polaron.

In Ref. (Mishchenko et al., 2000), the structure of the Fröhlich polaron cloud was investigated using the DQMC method. Contributions of $N$-phonon states to the polaron ground state were calculated as a function of $N$ for a few values of the coupling constant $\alpha$, see Fig 4 , lower panel. As follows from Fig, 4 , the evolution from the weak-coupling case $(\alpha=1)$ into the strong-coupling regime $(\alpha=17)$ was studied. The evolution of the shape and the scale of the distribution of the $N$-phonon states with increasing $\alpha$ as derived for a Fröhlich polaron within DQMC method (Mishchenko et al., 2000) is in notable agreement with the results obtained within the "symmetric" 1D polaron model (Devreese, 1964; Devreese and Evrard, 1964, 1968).

The insight gained as a result of those investigations concerning the structure of the excited polaron states, was subsequently used to develop a theory of the optical absorption spectra of polarons. The first work was limited to the strong coupling limit (Kartheuser et al. , 1969), where the impact of the internal degrees of freedom of polarons on their optical properties was revealed. The optical absorption of light by free Fröhlich polarons was treated in Ref. (Kartheuser et al. , 1969) using the polaron states obtained within the adiabatic strong-coupling approximation. It was argued in Ref. (Kartheuser et al. , 1969), that for sufficiently large $\alpha(\alpha \gtrsim 3)$, the (first) relaxed excited state (RES) of a polaron is 
The "symmetric" polaron model (Devreese 1964)
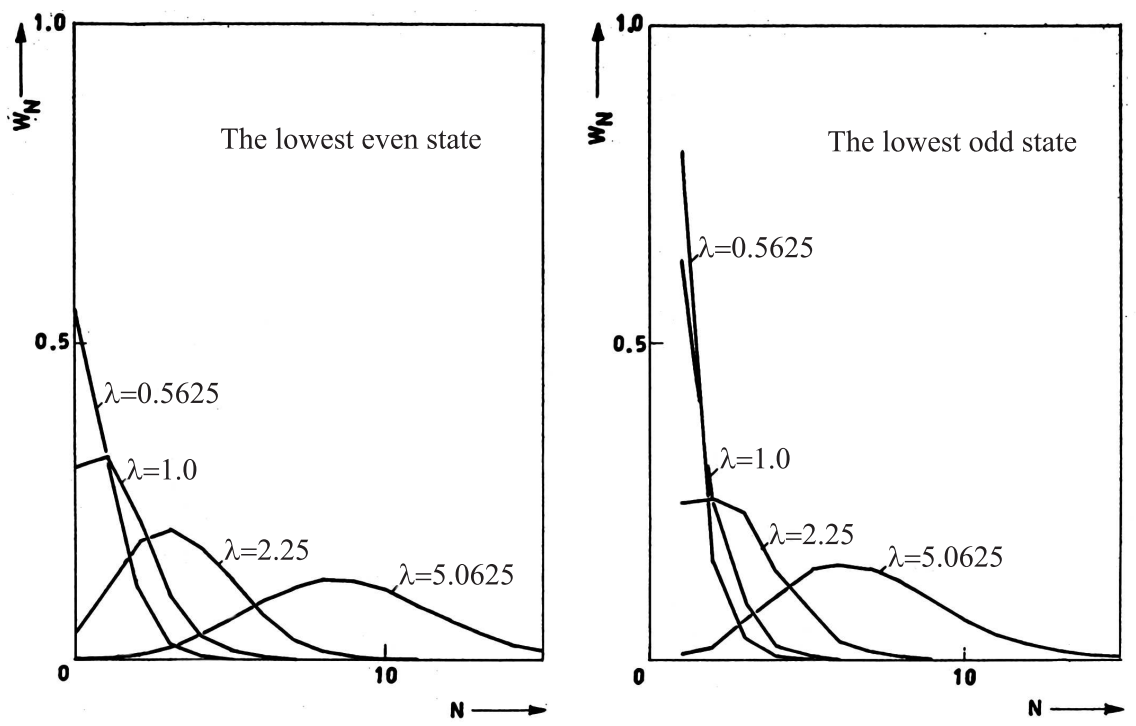

DQMC (Mishchenko et al. 2000)
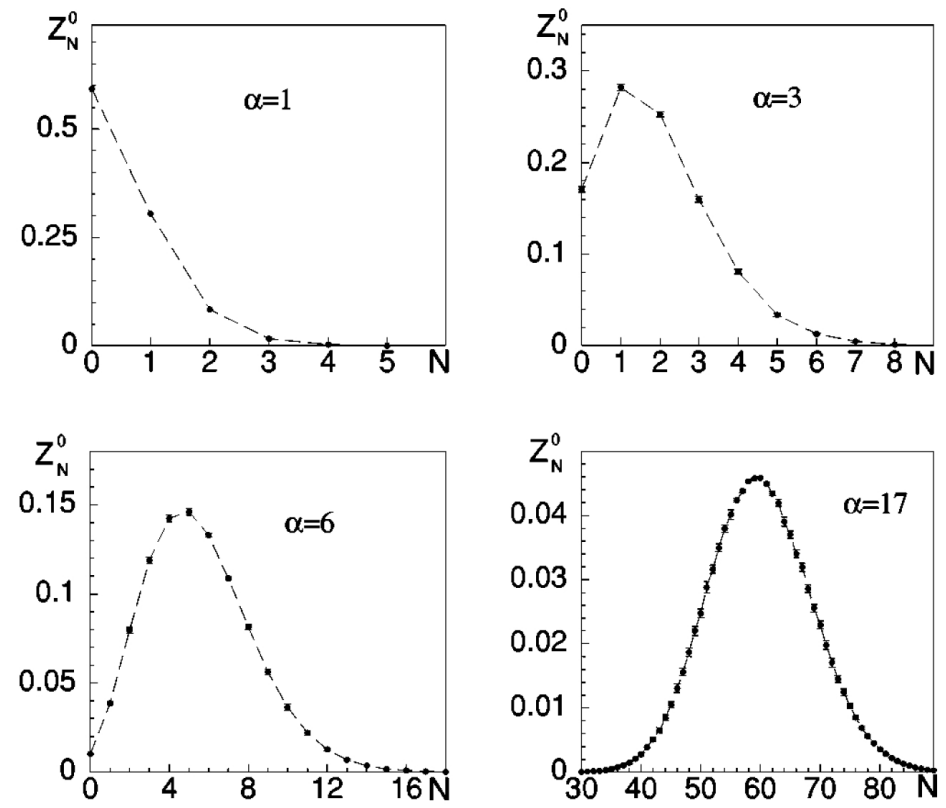

FIG. 4 Upper panel: The phonon distribution functions $W_{N}$ in the "symmetric" polaron model for various values of the effective coupling constant $\lambda$ at $\varkappa=1, \mathbf{P}=0$ (from (Devreese, 1964)). Lower panel: Distribution of multiphonon states in the polaron cloud within DQMC method for various values of $\alpha$ (from (Mishchenko et al., 2000)). (Reprinted with permission from (Devreese, 2006a). (C)2006, Società Italiana di Fisica.) 
a relatively stable state, which gives rise to a "resonance" in the polaron optical absorption spectrum. The following scenario of a transition, which leads to a "zero-phonon" peak in the absorption by a strong-coupling polaron, was then suggested. If the frequency of the incoming photon is equal to $\Omega_{\mathrm{RES}}=0.065 \alpha^{2} \omega_{0}$, the electron jumps from the ground state (which, at large coupling, is well-characterised by " $s$ "-symmetry for the electron) to an excited state (" $2 p ")$, while the lattice polarization in the final state is adapted to the " $2 p$ " electronic state of the polaron. In Ref. (Kartheuser et al. , 1969) considering the decay of the RES with emission of one real phonon, it is argued, that the "zero-phonon" peak can be described using the Wigner-Weisskopf formula which is valid when the linewidth of that peak is much smaller than $\omega_{0}$.

For photon energies larger than $\Omega_{\mathrm{RES}}+\omega_{\mathrm{LO}}$, a transition of the polaron towards the first scattering state, belonging to the RES, becomes possible. The final state of the optical absorption process then consists of a polaron in its lowest RES plus a free phonon. A "onephonon sideband" then appears in the polaron absorption spectrum. This process is called one-phonon sideband absorption. The one-, two-, ... K-, ... phonon sidebands of the zerophonon peak give rise to a broad structure in the absorption spectrum. It turns out that the first moment of the phonon sidebands corresponds to the Franck-Condon (FC) frequency $\Omega_{\mathrm{FC}}=0.141 \alpha^{2} \omega_{0}$.

To summarise, following (Kartheuser et al. , 1969), the polaron optical absorption spectrum at strong coupling is characterised by the following features (at $T=0)$ :

a) An absorption peak ("zero-phonon line") appears, which corresponds to a transition from the ground state to the first RES at $\Omega_{\mathrm{RES}}$.

b) For $\Omega>\Omega_{\mathrm{RES}}+\omega_{0}$, a phonon sideband structure arises. This sideband structure peaks around $\Omega_{\mathrm{FC}}$. Even when the zero-phonon line becomes weak, and most oscillator strength is in the LO-phonon sidebands, the zero-phonon line continues to determine the onset of the phonon sideband structure.

The basic qualitative strong coupling behaviour predicted in Ref. (Kartheuser et al. , 1969), namely, zero-phonon (RES) line with a broader sideband at the high-frequency side, was confirmed by later studies, as discussed below. 


\section{Optical absorption at arbitrary coupling}

The optical absorption of the Fröhlich polaron was calculated in 1972 (Devreese, 1972; Devreese et al. , 1972a) ("DSG") for the Feynman polaron model using path integrals. Until recently DSG combined with (Kartheuser et al. , 1969) constituted the basic picture for the optical absorption of the Fröhlich polaron. (Peeters and Devreese, 1983a) rederived the DSG-result using the memory function formalism (MFF). The DSG-approach is successful at weak electron-phonon coupling and is able to identify the excitations at intermediate electron-phonon coupling ( $3 \lesssim \alpha \lesssim 6$ ). In the strong coupling limit DSG still gives an accurate first moment for the polaron optical absorption but does not reproduce the broad phonon sideband structure (cf. (Kartheuser et al. , 1969) and (Goovaerts et al. 1973)). A comparison of the DSG results with the optical conductivity spectra given by recently developed "approximation-free" numerical (Mishchenko et al. , 2003) and approximate analytical (De Filippis et al. , 2003, 2006) approaches was carried out recently (De Filippis et al. 2006), see also the review articles (Cataudella et al., 2007) and (Mishchenko and Nagaosa, 2007).

The polaron absorption coefficient $\Gamma(\Omega)$ of light with frequency $\Omega$ at arbitrary coupling was first derived in Ref. (Devreese et al. 1972a). It was represented in the form

$$
\Gamma(\Omega)=-\frac{4 \pi}{n c} \frac{e^{2}}{m} \frac{\operatorname{Im} \Sigma(\Omega)}{[\Omega-\operatorname{Re} \Sigma(\Omega)]^{2}+[\operatorname{Im} \Sigma(\Omega)]^{2}} .
$$

This general expression was the starting point for a derivation of the theoretical optical absorption spectrum of a single Fröhlich polaron at all electron-phonon coupling strengths by (Devreese et al. , 1972a) . $\Sigma(\Omega)$ is the so-called "memory function", which contains the dynamics of the polaron and depends on $\Omega, \alpha$, temperature and applied external fields. The key contribution of (Devreese et al. , 1972a) was to introduce $\Gamma(\Omega)$ in the form (111) and to calculate $\operatorname{Re} \Sigma(\Omega)$, which is essentially a (technically not trivial) Kramers-Kronig transform of the more simple function $\operatorname{Im} \Sigma(\Omega)$. Only the function $\operatorname{Im} \Sigma(\Omega)$ had been derived for the Feynman polaron (Feynman et al. , 1962) to study the polaron mobility $\mu$ using the impedance function (82): $\mu^{-1}=\lim _{\Omega \rightarrow 0}(\operatorname{Im} \Sigma(\Omega) / \Omega)$.

The basic nature of the Fröhlich polaron excitations was clearly revealed through this polaron optical absorption given by Eq. (111). It was demonstrated (Devreese et al. , 1972a) that the FC states for Fröhlich polarons are nothing else but a superposition of 
phonon sidebands and a relatively large value of the electron-phonon coupling strength $(\alpha>5.9)$ is needed to stabilise the relaxed excited state of the polaron. It was, further, revealed that at weaker coupling only "scattering states" of the polaron play a significant role in the optical absorption (Devreese et al. $, 1971,1972 \mathrm{a})$.

In the weak coupling limit, the absorption spectrum (111) of the polaron is determined by the absorption of radiation energy, which is re-emitted in the form of LO phonons. As $\alpha$ increases between approximately 3 and 6 , a resonance with increasing stability appears in the optical absorption of the Fröhlich polaron of Ref. (Devreese et al. , 1972a) (see Fig. 5). The RES peak in the optical absorption spectrum also has a phonon sideband-structure, whose average transition frequency can be related to a FC-type transition. Furthermore, at zero temperature, the optical absorption spectrum of one polaron exhibits also a zerofrequency "central peak" $[\propto \delta(\Omega)]$. For nonzero temperature, this "central peak" smears out and gives rise to an "anomalous" Drude-type low-frequency component of the optical absorption spectrum.

For $\alpha>6.5$ the polaron optical absorption gradually develops the structure qualitatively proposed in (Kartheuser et al. , 1969): a broad LO-phonon sideband structure appears with
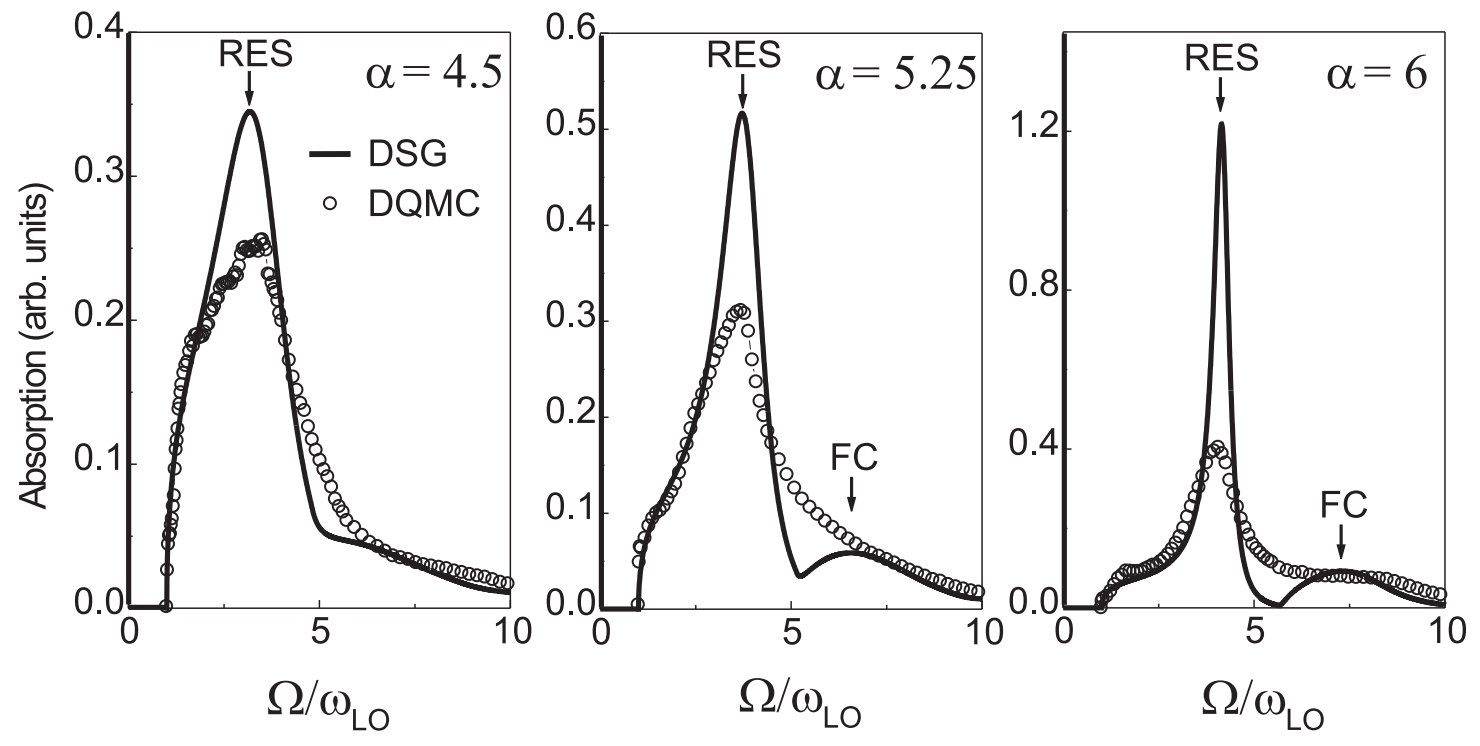

FIG. 5 Optical absorption spectrum of a Fröhlich polaron for $\alpha=4.5, \alpha=5.25$ and $\alpha=6$ after Ref. (Devreese et al. , 1972a) (DSG). The RES peak is very intense compared with the FC peak. The $\delta$-like central peaks (at $\Omega=0$ ) are schematically shown by vertical lines. The DQMC results of Ref. (Mishchenko et al. , 2003) are shown with open circles. 
the zero-phonon ("RES") transition as onset. Ref. (Devreese et al. , 1972a) does not predict the broad LO-phonon sidebands at large coupling constant, although it still gives an accurate first Stieltjes moment of the optical absorption spectrum.

Based on (Devreese et al. , 1972a), it was argued that it is rather Holstein polarons that determine the optical properties of the charge carriers in oxides like $\mathrm{SrTiO}_{3}, \mathrm{BaTiO}_{3}$ (Huybrechts and Devreese, 1975), while Fröhlich weak-coupling polarons could be identified e. g. in CdO (Devreese et al. , 1971). The Fröhlich coupling constants of polar semiconductors and ionic crystals are generally too small to allow for a static "RES". In Ref. (Eagles et al., 1995) the RES-peaks of Ref. (Devreese et al. , 1972a) were invoked to explain the optical absorption spectrum of $\mathrm{Pr}_{2} \mathrm{NiO}_{4.22}$. The RES-like resonances in $\Gamma(\Omega)$, Eq. (111), due to the zero's of $\Omega-\operatorname{Re} \Sigma(\Omega)$, can effectively be displaced to smaller polaron coupling by applying an external magnetic field $B$, in which case the contribution for what is formally a "RES-type resonance" arises at $\Omega-\omega_{c}-\operatorname{Re} \Sigma(\Omega)=0\left(\omega_{c}=e B / m\right.$ is the cyclotron frequency). Resonances in the magnetoabsorption governed by this contribution have been clearly observed and analysed in many solids and structures, see e. g. (Devreese, 1996, 2003; Hodby et al., 1987; Miura et al. , 1997; Peeters and Devreese,, 1986; Shi et al. , 1993) and references therein.

Evidence for the polaron character of charge carriers in $\mathrm{AgBr}$ and $\mathrm{AgCl}$ was obtained through high-precision cyclotron resonance experiments in external magnetic fields up to 16 T. The all-coupling magneto-absorption calculated in (Peeters and Devreese, 1986) leads to the best quantitative agreement between theory and experiment for $\mathrm{AgBr}$ and $\mathrm{AgCl}$. This quantitative interpretation of the cyclotron resonance experiment in $\mathrm{AgBr}$ and $\mathrm{AgCl}$ (Hodby et al. , 1987) by the theory of (Peeters and Devreese, 1986) provided one of the most convincing and clearest demonstrations of Fröhlich polaron features in solids. The energy spectra of polaronic systems such as shallow donors ("bound polarons"), e. g., the $\mathrm{D}_{0}$ and $\mathrm{D}^{-}$centres, constitute the most complete and detailed polaron spectroscopy realised in the literature (Shi et al. , 1993).

The numerical calculations of the optical conductivity for the Fröhlich polaron performed within the DQMC method (Mishchenko et al. , 2003) confirm the analytical results derived in Ref. (Devreese et al. , 1972a) for $\alpha \lesssim 3$. In the intermediate coupling regime $3<\alpha<6$, the low-energy behaviour and the position of the RES-peak in the optical conductivity spectrum of (Mishchenko et al. , 2003) follow closely the prediction of Ref. (Devreese et al. 
1972a). There are some minor quantitative differences between the two approaches in the intermediate coupling regime: the dominant ("RES") peak is less intense in the Monte-Carlo numerical simulations and the second ("FC") peak develops less prominently. The following qualitative differences exist between the two approaches: in (Mishchenko et al. , 2003), the dominant peak broadens for $\alpha \gtrsim 6$ and the second peak does not develop, but gives rise to a flat shoulder in the optical conductivity spectrum at $\alpha \approx 6$. As $\alpha$ increases beyond $\alpha \approx 6$, the DSG results for the OC do not produce the broad phonon sideband spectrum of the RES-transition that was qualitatively predicted in Ref. (Kartheuser et al. , 1969) and obtained with DQMC.

An instructive comparison between the positions of the main peak in the optical absorption spectra of Fröhlich polarons obtained within the DSG and DQMC approaches has been

performed recently (Devreese and Klimin , 2006). The main-peak positions, obtained within DSG, have been found in good agreement with the results of DQMC for all considered values of $\alpha$. At large $\alpha$ the positions of the main peak in the DSG spectra are remarkably close to those given by DQMC. The difference between the DSG and DQMC results is relatively larger at $\alpha=8$ and for $\alpha=9.5$, but even for those values of the coupling constant the agreement is rather good.

It is suggested that the RES-peak at $\alpha \approx 6$ in the DSG-treatment, as $\alpha$ increases, gradually transforms onto a FC-peak. As stated above and in Ref. (Devreese et al. , 1972a), DSG predicts a much too narrow FC-peak in the strong coupling limit, but still at the "correct" frequency. The DSG spectrum also satisfies the zero and first moment sum rules at all $\alpha$ as discussed below.

\section{E. Main-peak line and strong-coupling expansion}

In order to describe the OC main peak line width at intermediate electron-phonon coupling, the DSG approach was modified (De Filippis et al. , 2006) to include additional dissipation processes, the strength of which is fixed by an exact sum rule (Cataudella et al., 2007).

Within the memory function formalism (MFF) (Götze and P. Wolfle, 1972; Mori , 1965) the interaction of the charge carriers with the free phonon oscillations is expressed in terms 
of the electron density-density correlation function,

$$
\chi(\mathbf{q}, t)=-i \Theta(t)\langle\exp [i \mathbf{q} \cdot \mathbf{r}(t)] \exp [-i \mathbf{q} \cdot \mathbf{r}(0)]\rangle,
$$

which is evaluated in a direct way (Peeters and Devreese, 1983a) using the Feynman polaron model, where the electron is coupled via a harmonic force to a fictitious particle that simulates the phonon degrees of freedom. Within this procedure the electron density-density correlation function takes the form:

$$
\chi_{m}(\mathbf{q}, t)=-i \Theta(t) \exp \left[-i q^{2} t / 2 M\right] \exp \left[-q^{2} R\left(1-e^{-i v t}\right) / 2 M\right]
$$

where $R=(M-1) / v, M$ (the total mass of electron and fictitious particle), and $v$ are determined variationally within the path integral approach (Fevnman, 1955). The associated spectral function $A_{m}(\mathbf{q}, \omega)=-2 \operatorname{Im} \chi_{m}(\mathbf{q}, \omega)$ is a series of $\delta$ functions centered at $q^{2} / 2 M+n v$ ( $n$ is integer). Here $q^{2} / 2 M$ represents the energy of the center of mass of electron and fictitious particle, and $v$ is the energy gap between the levels of the relative motion. To include dissipation (De Filippis et al. , 2006), a finite lifetime was introduced for the states of the relative motion, which can be considered as the result of the residual EPI not included into the Feynman variational model. To this end, in $\chi_{m}(\mathbf{q}, t)$ the factor exp [-ivt] was replaced with $(1+i t / \tau)^{-v \tau}$ which leads to the replacement of $\delta$ functions by Gamma functions with mean value and variance given respectively by $q^{2} / 2 M+n v$ and $n v / \tau$. The parameter of dissipation $\tau$ is determined by the third sum rule for $A(\mathbf{q}, \omega)$, which is additional to the first two sum rules that are already satisfied in the DSG model without damping. As expected, $\tau$ turns out to be of the order of $\omega_{0}^{-1}$. If broadening of the oscillator levels is neglected, the DSG results (Devreese et al. , 1972a; Peeters and Devreese, 1983a) are recovered.

Starting from the Kubo formula, the strong-coupling polaron optical conductivity can be evaluated using the strong-coupling expansion (SCE), Refs. (Cataudella et al., 2007; De Filippis et al. , 2006). In Refs. (Devreese, 2007a, b); Devreese and Klimin , 2006) SCE has been extended. In order to apply the extended SCE for the polaron OC, a scaling transformation of the coordinates and moments of the electron-phonon system is made following Allcock (Allcock, 1963) $, \mathbf{r}=\alpha^{-1} \mathbf{x}, \mathbf{p}=-i \alpha \partial / \partial \mathbf{x}$, and $\mathbf{q}=\alpha \tilde{\mathbf{q}}$. This transformation allows us to see explicitly the order of magnitude of different terms in the Hamiltonian. Expressed in terms of the new variables, the Hamiltonian can be written as a sum of two terms, which are of different orders in powers of $\alpha, H=H_{1}+H_{2}$, where $H_{1} \sim \alpha^{2}$ is the leading term, and 
$H_{2} \sim \alpha^{-2}$ is the kinetic energy of the vibrating ions. The next step is the Born-Oppenheimer approximation (Allcock, 1963), which neglects the non-adiabatic transitions between different polaron levels in calculating the dipole-dipole correlation function of the Kubo formula (Devreese, 2007b; Devreese and Klimin, 2006).

Figure 6] shows the polaron OC spectra for different values of $\alpha$ calculated numerically using the extended SCE with different approximations. The OC spectra calculated within the extended SCE approach, taking into account both the Jahn-Teller effect and the corrections of order $\alpha^{0}$, are shown by the solid curves. The OC obtained with the leadingterm strong-coupling approximation taking into account the Jahn-Teller effect and with the leading term of the Landau-Pekar adiabatic approximation are plotted as dashed and dashdotted curves, respectively. The full circles show the DQMC data (De Filippis et al. , 2006; Mishchenko et al. , 2003).

The polaron OC band of Fig. [6 obtained within the extended SCE generalises the Gaussian-like polaron OC band (as given e.g. by Eq. (3) of Ref.(De Filippis et al. 2006)) thanks to (i) the use of the numerically exact strong-coupling polaron wave functions (Miyake, 1975) and (ii) the incorporation of both static and dynamic Jahn-Teller effects. The polaron OC broad structure calculated within the extended SCE consists of a series of LO-phonon sidebands and provides a realisation — with all LO-phonons involved for a given $\alpha$ - of the strong-coupling scheme proposed in (Kartheuser et al. , 1969).

As seen from Fig. 6, the polaron OC spectra calculated within the asymptotically exact strong-coupling approach are shifted towards lower frequencies as compared with the OC spectra calculated within the LP approximation. This shift is due to the use of the numerically exact (in the strong-coupling limit) energy levels and wave functions of the internal excited polaron states, as well as the numerically exact self-consistent adiabatic polaron potential. Furthermore, the inclusion of the corrections of order $\alpha^{0}$ leads to a shift of the OC spectra to lower frequencies with respect to the OC spectra calculated within the leadingterm approximation. The value of this shift $\Delta \Omega_{n, 0} / \omega_{\mathrm{L} O} \approx-1.8$ obtained in the present calculation, is close to the LP value $\Delta \Omega_{n, 0}^{(L P)} / \omega_{0}=4 \ln 2-1 \approx 1.7726$ (cf. (Allcock, 1956; Feynman, 1972)). The distinction between the OC spectra calculated with and without the Jahn-Teller effect is very small.

Starting from $\alpha \approx 9$ towards larger values of $\alpha$, the agreement between the extended SCE polaron OC spectra and the numerical DQMC data becomes gradually better, consistent 


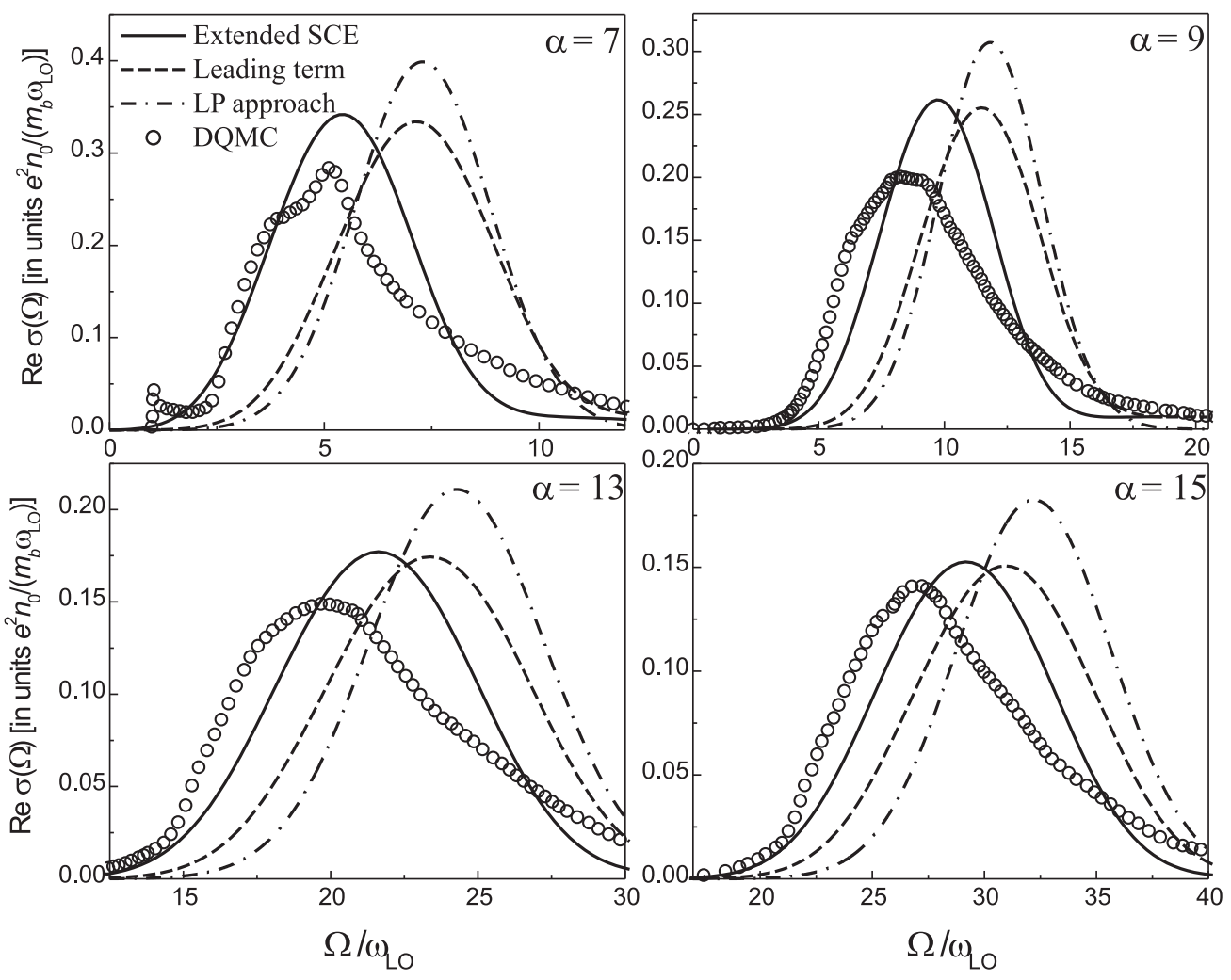

FIG. 6 The polaron OC calculated within the extended SCE taking into account corrections of order $\alpha^{0}$ (solid curve), the OC calculated within the leading-term strong-coupling approximation (dashed curve), with the leading term of the Landau-Pekar (LP) adiabatic approximation (dashdotted curve), and the numerical DQMC data (open circles) for $\alpha=7,9,13$ and 15. (From Ref. (Devreese and Klimin, 2006).)

with the fact that the extended SCE for the polaron OC is asymptotically correct in the strong-coupling limit. The results of the extended SCE are qualitatively consistent with the interpretation advanced in Ref. (Kartheuser et al. , 1969). In (Kartheuser et al. , 1969) only the 1-LO-phonon sideband was taken into account, while in Ref. (Goovaerts et al. , 1973) 2-LO-phonon emission was included. The extended SCE carries on the program started in Ref. (Kartheuser et al. , 1969). The spectra in Fig. 6, in the strong coupling approximation, consist of LO-phonon sidebands to the RES (which itself has negligible oscillator strength in this limit, similar to the optical absorption for some colour centres in alkali halides). These LO-phonon sidebands form a broad FC-structure. 


\section{F. Comparison between optical conductivity spectra obtained by different methods}

A comparison between the optical conductivity spectra obtained with the DQMC method, extended MFF, SCE and DSG for different values of $\alpha$ is shown in Figs. 7, 8, taken from Ref. (De Filippis et al. , 2006). The key results of the comparison are the following.

First, as expected, in the weak-coupling regime, both the extended MFF with phonon broadening and DSG (Devreese et al. , 1972a) are in very good agreement with the DQMC data (Mishchenko et al. , 2003), showing significant improvement with respect to the weakcoupling perturbation approach (Devreese et al. , 1971; Gurevich et al. , 1962) which provides a good description of the OC spectra only for very small values of $\alpha$. For $3 \leq \alpha \leq 6$, DSG predicts the essential structure of the optical absorption, with a RES-transition gradually building up for increasing $\alpha$, but underestimates the peak width. The damping, introduced in the extended MFF approach, becomes crucial in this coupling regime.

Second, comparing the peak and shoulder energies, obtained by DQMC, with the peak energies, given by MFF, and the FC transition energies from the SCE, it is concluded (De Filippis et al. , 2006) that as $\alpha$ increases from 6 to 10 the spectral weights rapidly switch from the dynamic regime, where the lattice follows the electron motion, to the adiabatic regime dominated by $\mathrm{FC}$ transitions. In the intermediate electron-phonon coupling regime,
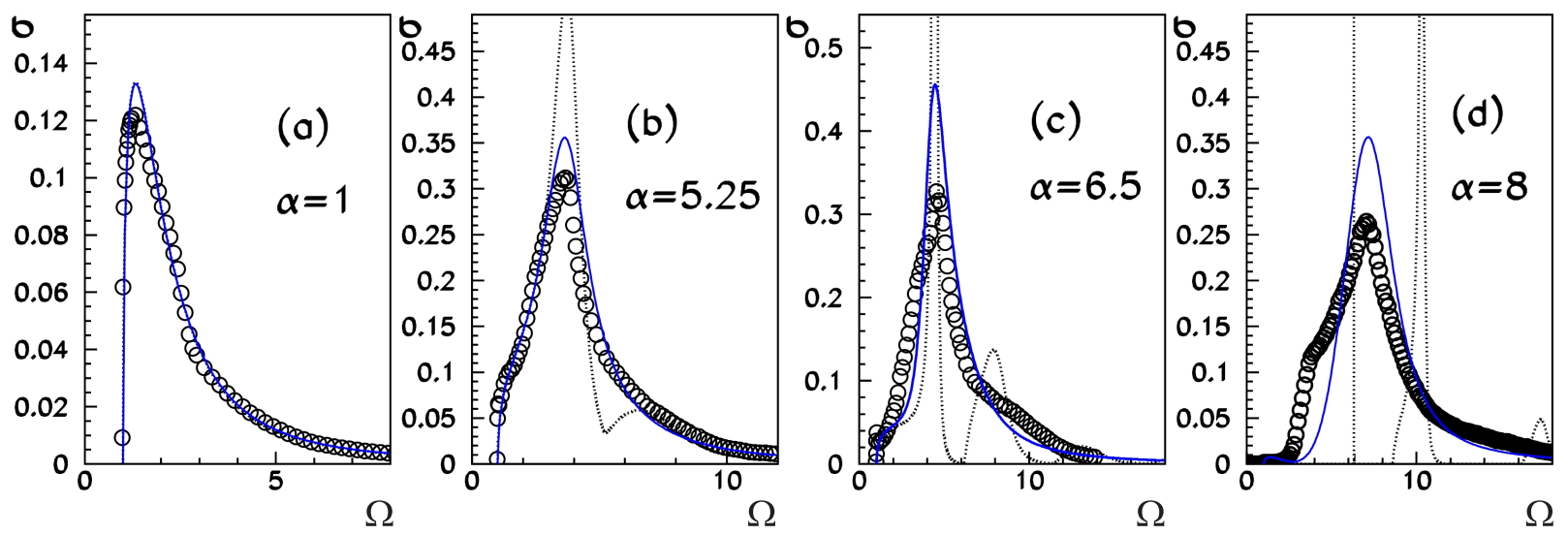

FIG. 7 Comparison of the optical conductivity calculated with the DQMC method (circles), extended MFF (solid line) and DSG (Devreese et al. , 1972a; Peeters and Devreese, 1983a) (dotted line), for four different values of $\alpha$. The arrow indicates the lower-frequency feature in the DQMC data. (Reprinted with permission from Ref. (De Filippis et al. , 2006). (C)2006 by the American Physical Society.) 
$6<\alpha<10$, both adiabatic FC and non-adiabatic dynamical excitations coexist. For still larger coupling $\alpha \gtrsim 10$, the absorption spectrum consists of a broad FC-structure, built of LO-phonon sidebands.

In summary, the accurate numerical results obtained from DQMC and from the recent analytical approximations (De Filippis et al. , 2003, 2006) confirm the essence of the mechanism for the optical absorption of Fröhlich polarons, proposed in Refs. (Devreese, 1972; Devreese et al. , 1972a) combined with (Kartheuser et al. , 1969) and do add important new insights.

\section{G. Sum rules for the optical conductivity spectra of Fröhlich polarons}

In this section several sum rules for the optical conductivity spectra of Fröhlich polarons are applied to test the DSG approach (Devreese et al. , 1972a) and the DQMC results (Mishchenko et al. , 2003). The values of the polaron effective mass for the DQMC approach are taken from Ref. (Mishchenko et al., 2000). In Tables II and III, we show the polaron
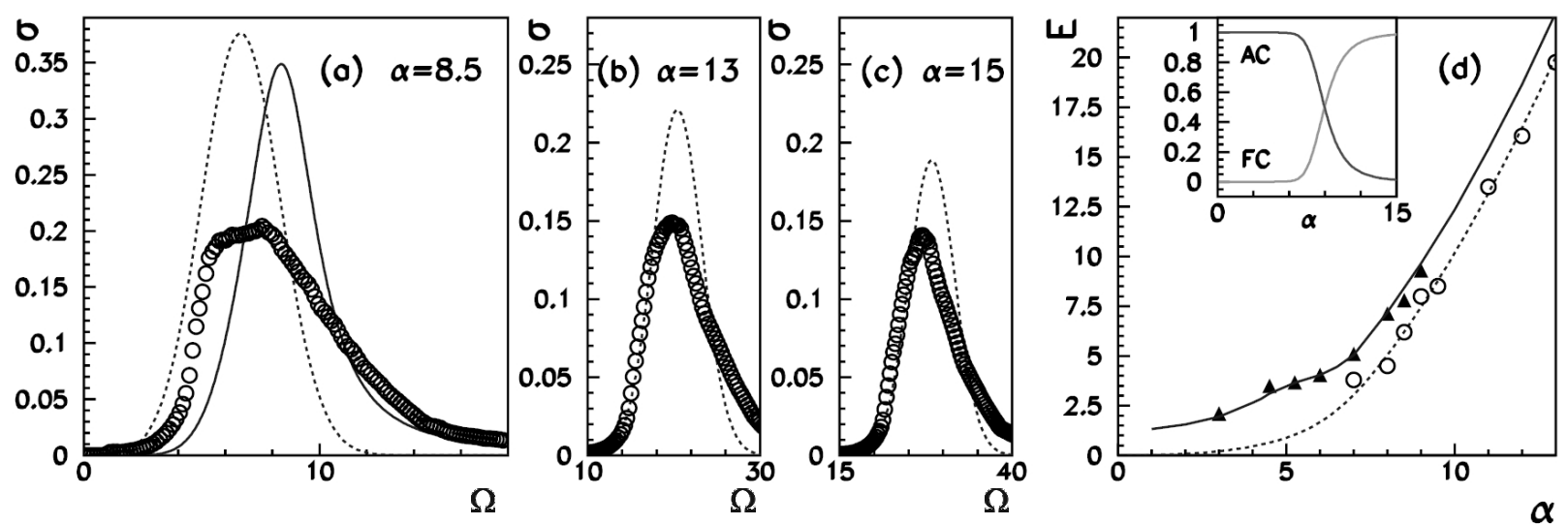

FIG. 8 a), b) and c) Comparison of the optical conductivity calculated with the DQMC method (circles), the extended MFF(solid line) and SCE (dashed line) for three different values of $\alpha . \mathrm{d}$ ) The energy of the lower- and higher-frequency features obtained by DQMC (circles and triangles, respectively) compared (i) with the FC transition energy calculated from the SCE (dashed line) and (ii) with the energy of the peak obtained from the extended MFF (solid line). In the inset the weights of Franck-Condon and adiabatically connected transitions are shown as a function of $\alpha$. We have used for $\eta$ the value 1.3. (Reprinted with permission from Ref. (De Filippis et al. , 2006). (C)2006 by the American Physical Society.) 
ground-state $E_{0}$ and the zero, $M_{0}$ and first, $M_{1}$, frequency moments calculated using the optical conductivity spectra:

$$
\begin{aligned}
& M_{0} \equiv \int_{1}^{\Omega_{\max }} \operatorname{Re} \sigma(\Omega) d \Omega \\
& M_{1} \equiv \int_{1}^{\Omega_{\max }} \Omega \operatorname{Re} \sigma(\Omega) d \Omega,
\end{aligned}
$$

where $\Omega_{\max }$ is the upper value of the frequency available from Ref. (Mishchenko et al. 2003), and

$$
\tilde{M}_{0} \equiv \frac{\pi}{2 m^{*}}+\int_{1}^{\Omega_{\max }} \operatorname{Re} \sigma(\Omega) d \Omega .
$$

Here $m^{*}$ is the polaron mass, the optical conductivity is calculated in units $n_{p} e^{2} /\left(m \omega_{0}\right), m^{*}$ is measured in units of the band mass $m$, and the frequency is measured in units of $\omega_{0}$. The values of $\Omega_{\max }$ are: $\Omega_{\max }=10$ for $\alpha=0.01,1$ and $3, \Omega_{\max }=12$ for $\alpha=4.5,5.25$ and 6 , $\Omega_{\max }=18$ for $\alpha=6.5,7$ and 8 .

TABLE II Polaron parameters $M_{0}, M_{1}, \tilde{M}_{0}$ obtained from the diagrammatic Monte Carlo results (Reprinted with permission from (Devreese, 2006a). (C)2006, Società Italiana di Fisica.)

\begin{tabular}{|l|l|l|l|l|l|}
\hline \hline$\alpha$ & $M_{0}^{(\mathrm{DQMC})}$ & $m^{*(\mathrm{DQMC})}$ & $\tilde{M}_{0}^{(\mathrm{DQMC})}$ & $M_{1}^{(\mathrm{DQMC})} / \alpha$ & $E_{0}^{(\mathrm{DQMC})}$ \\
\hline 0.01 & 0.00249 & 1.0017 & 1.5706 & 0.634 & -0.010 \\
1 & 0.24179 & 1.1865 & 1.5657 & 0.65789 & -1.013 \\
3 & 0.67743 & 1.8467 & 1.5280 & 0.73123 & -3.18 \\
4.5 & 0.97540 & 2.8742 & 1.5219 & 0.862 & -4.97 \\
5.25 & 1.0904 & 3.8148 & 1.5022 & 0.90181 & -5.68 \\
6 & 1.1994 & 5.3708 & 1.4919 & 0.98248 & -6.79 \\
6.5 & 1.30 & 6.4989 & 1.5417 & 1.1356 & -7.44 \\
7 & 1.3558 & 9.7158 & 1.5175 & 1.2163 & -8.31 \\
8 & 1.4195 & 19.991 & 1.4981 & 1.3774 & -9.85 \\
\hline \hline
\end{tabular}

The optical conductivity derived by DSG (Devreese et al. , 1972a) exactly satisfies the 
TABLE III Polaron parameters $M_{0}, M_{1}, \tilde{M}_{0}$ obtained within the path-integral approach (Reprinted with permission from (Devreese, 2006a). (C)2006, Società Italiana di Fisica.)

\begin{tabular}{|l|l|l|l|l|l|}
\hline \hline$\alpha$ & $M_{0}^{(\mathrm{DSG})}$ & $m^{* \text { (Feynman })}$ & $\tilde{M}_{0}^{(\mathrm{DSG})}$ & $M_{1}^{(\mathrm{DSG})} / \alpha$ & $E_{0}^{\text {(Feynman })}$ \\
\hline 0.01 & 0.00248 & 1.0017 & 1.5706 & 0.633 & -0.010 \\
1 & 0.24318 & 1.1957 & 1.5569 & 0.65468 & -1.0130 \\
3 & 0.69696 & 1.8912 & 1.5275 & 0.71572 & -3.1333 \\
4.5 & 1.0162 & 3.1202 & 1.5196 & 0.83184 & -4.8394 \\
5.25 & 1.1504 & 4.3969 & 1.5077 & 0.88595 & -5.7482 \\
6 & 1.2608 & 6.8367 & 1.4906 & 0.95384 & -6.7108 \\
6.5 & 1.3657 & 9.7449 & 1.5269 & 1.1192 & -7.3920 \\
7 & 1.4278 & 14.395 & 1.5369 & 1.2170 & -8.1127 \\
8 & 1.4741 & 31.569 & 1.5239 & 1.4340 & -9.6953 \\
\hline \hline
\end{tabular}

sum rule (Devreese et al., 1977$)$

$$
\frac{\pi}{2 m^{*}}+\int_{1}^{\infty} \operatorname{Re} \sigma(\Omega) d \Omega=\frac{\pi}{2}
$$

Since the optical conductivity obtained from the DQMC results (Mishchenko et al. , 2003) is known only within a limited interval of frequencies $1<\Omega<\Omega_{\max }$, the integral in Eq. (116) for the DSG-approach (Devreese et al. , 1972a) is calculated over the same frequency interval as for the Monte Carlo results (Mishchenko et al. , 2003).

The comparison of the resulting zero frequency moments $\tilde{M}_{0}^{(\mathrm{DQMC})}$ and $\tilde{M}_{0}^{(\mathrm{DSG})}$ with each other and with the value $\pi / 2=1.5707963 \ldots$ corresponding to the right-hand-side of the sum rule (117) shows that the difference $\left|\tilde{M}_{0}^{(\mathrm{DQMC})}-\tilde{M}_{0}^{(\mathrm{DSG})}\right|$ on the interval $\alpha \leq 8$ is smaller than the absolute value of the contribution of the "tail" of the optical conductivity for $\Omega>\Omega_{\max }$ to the integral in the sum rule (117):

$$
\int_{\Omega_{\max }}^{\infty} \operatorname{Re} \sigma^{(\mathrm{DSG})}(\Omega) d \Omega \equiv \frac{\pi}{2}-\tilde{M}_{0}^{(\mathrm{DSG})} .
$$

Within the accuracy determined by the neglect of the "tail" of the part of the spectrum for $\Omega>\Omega_{\max }$, the contribution to the integral in the sum rule (117) for the optical conductivity obtained from the DQMC results (Mishchenko et al. , 2003) agrees well with that for 
the optical conductivity found within the path-integral approach in Ref. (Devreese et al. , 1972a). Hence, the conclusion follows that the optical conductivity obtained from the DQMC results (Mishchenko et al. , 2003) satisfies the sum rule (117) within the aforementioned accuracy.

\section{H. Optical absorption spectra of continuum-polaron gas}

For the weak-coupling regime, which is realized in most polar semiconductors, the groundstate energy of a gas of interacting continuum polarons has been derived in (Lemmens et al., 1977) by introducing a variational wave function:

$$
\left|\psi_{\mathrm{LDB}}\right\rangle=U|\phi\rangle\left|\varphi_{\mathrm{el}}\right\rangle
$$

where $\left|\varphi_{\mathrm{el}}\right\rangle$ represents the ground-state many-body wave function for the electron (or hole) system, $|\phi\rangle$ is the phonon vacuum and $U$ is a many-body unitary operator. $U$ defines the LDB-canonical transformation for a fermion gas interacting with a boson field:

$$
U=\exp \left\{\sum_{j=1}^{N} \sum_{\mathbf{q}}\left(f_{\mathbf{q}} d_{\mathbf{q}} e^{i \mathbf{q} \cdot \mathbf{r}_{j}}-f_{\mathbf{q}}^{*} d_{\mathbf{q}}^{+} e^{-i \mathbf{q} \cdot \mathbf{r}_{j}}\right)\right\}
$$

where $\mathbf{r}_{j}$ represent the position of the $N$ constituent electrons (or holes). The $f_{\mathbf{q}}$ were determined variationally (Lemmens et al., 1977). It may be emphasized that Eq. (120), although it appears like a straightforward generalization of the one-particle transformation in (Tomonaga, 1947), constitutes - especially in its implementation - a nontrivial extension of a one-particle approximation to a many-body system. An advantage of the LDB-manypolaron canonical transformations introduced in (Lemmens et al., 1977) for the calculation of the ground state energy of a polaron gas is that the many-body effects are contained in the static structure factor of the electron (or hole) system, which appears in the analytical expression for the energy. Within the approach the minimum of the total ground-state energy per particle for a polaron gas lies at lower density than that for the electron gas.

The non-degenerate system of interacting polarons in polar doped insulators was analysed by Fratini and Quémerais (2000) using a simplified Feynman-type polaron model. In the low-density limit, the ground state of the many-polaron system is the Wigner lattice of polarons. With increasing density depending on the value of $\alpha$, one of the following two 
scenarios is possible: (i) the melting of the polaron Wigner lattice for $\alpha<\alpha^{*}$ and (ii) the dissociation of the polarons for $\alpha>\alpha^{*}$ (Fratini and Quémerais, 2000).

The LDB-canonical transformation has been fruitfully applied in the theory of optical absorption spectra of many-polaron systems. In (Tempere and Devreese, 2001), starting from the LDB-many-polaron canonical transformation and the variational many-polaron wave function introduced in (Lemmens et al., 1977), the optical absorption coefficient of a many-polaron gas has been derived. The real part of the optical conductivity of the many-polaron system is obtained in an intuitively appealing form, given by Eq. (107).

This approach to the many-polaron optical absorption allows one to include the manybody effects to order $\alpha$ in terms of the dynamical structure factor $S\left(\mathbf{k}, \Omega-\omega_{\mathrm{LO}}\right)$ of the electron (or hole) system. The experimental peaks in the mid-infrared optical absorption spectra of cuprates (Lupi et al., 1999), and manganites (Hartinger et al., 2004) have been adequately interpreted within this theory. The many-polaron approach describes the experimental optical conductivity better than the single-polaron approximations (Emin, 1993; Gurevich et al. , 1962). Note that in (Tempere and Devreese, 2001), like in (Eagles et al., 1995), coexistence of small and Fröhlich polarons in the same solid is involved.

The optical conductivity of a many-polaron gas was investigated in (Cataudella et al.), 1999) in a different way by calculating the correction to the dielectric function of the electron gas, due to the electron-phonon interaction with variational parameters of a single-polaron Feynman model. A suppression of the optical absorption of a many-polaron gas as compared to the one-polaron optical absorption of Refs. (Devreese, 1972; Devreese et al. , 1972a) with increasing density has been found. Such a suppression is expected because of the screening of the Fröhlich interaction with increasing polaron density.

\section{Ripplopolarons}

An interesting 2D system consists of electrons on films of liquid He (Jackson and Platzman, 1981; Shikin and Monarkha, 1973). In this system the electrons couple to the ripplons of the liquid He, forming "ripplopolarons". The effective coupling can be relatively large and self-trapping can result. The acoustic nature of the ripplon dispersion at long wavelengths induces the self-trapping. Spherical shells of charged particles appear in a variety of physical systems, such as fullerenes, metallic nanoshells, 
charged droplets and neutron stars. A particularly interesting physical realization of the spherical electron gas is found in multielectron bubbles (MEBs) in liquid helium-4. These MEBs are $0.1 \mu \mathrm{m}-100 \mu \mathrm{m}$ sized cavities inside liquid helium, that contain helium vapour at vapour pressure and a nanometer-thick electron layer, anchored to the surface of the bubble (Albrecht and Leiderer, 1987; Volodin et al., 1977). They exist as a result of equilibrium between the surface tension of liquid helium and the Coulomb repulsion of the electrons (Salomaa and Williams, 1981; Shikin, 1978).

Recently proposed experimental schemes to stabilize MEBs (Silvera, 2001) have stimulated theoretical investigation of their properties (see e. g. (Tempere et al., 2001)). The dynamical modes of MEB were described by considering the motion of the helium surface ("ripplons") and the vibrational modes of the electrons together. In particular, the case when the ripplopolarons form a Wigner lattice was analyzed. The interaction energy between the ripplons and the electrons in the multielectron bubble is derived from the following considerations: (i) the distance between the layer electrons and the helium surface is fixed (the electrons find themselves confined to an effectively 2D surface anchored to the helium surface) and (ii) the electrons are subjected to a force field, arising from the electric field of the other electrons. To study the ripplopolaron Wigner lattice at nonzero temperature and for any value of the electron-ripplon coupling, the variational path-integral approach (Feynman, 1955) has been used. The destruction of the ripplopolaron Wigner lattice in a MEB occurs through the dissociation of ripplopolarons. Below a critical pressure (on the order of $10^{4} \mathrm{~Pa}$ ) the ripplopolaron solid will melt into an electron liquid. This critical pressure is nearly independent of the number of electrons (except for the smallest bubbles) and is weakly temperature dependent, up to the helium critical temperature $5.2 \mathrm{~K}$. This can be understood since the typical lattice potential well in which the ripplopolaron resides has frequencies of the order of $\mathrm{THz}$ or larger, which correspond to $\sim 10 \mathrm{~K}$.

The new phase that was predicted in (Tempere et al., 2003a), the ripplopolaron Wigner lattice, will not be present for electrons on a flat helium surface. At the values of the pressing field necessary to obtain a strong enough electron-ripplon coupling, the flat helium surface is no longer stable against long-wavelength deformations (Gor'kov and Chernikova, 1973). Multi-electron bubbles, with their different ripplon dispersion and the presence of stabilizing factors such as the energy barrier against fissioning (Tempere et al., 2003b), allow for much larger electric fields pressing the electrons against the helium surface. The regime of $N, p, T$ 
parameters suitable for the creation of a ripplopolaron Wigner lattice lies within the regime that would be achievable in recently proposed experiments, aimed at stabilizing multielectron bubbles (Silvera, 2001). The ripplopolaron Wigner lattice and its melting transition might be

detected by spectroscopic techniques (Fisher et al., 1979; Grimes and Adams, 1979) probing for example the transverse phonon modes of the lattice (Deville et al., 1984).

\section{J. Polaron scaling relations}

The form of the Fröhlich Hamiltonian, Eq.(28), in $n$ dimensions is the same as in 3D, except that now all vectors are $n$-dimensional. In this subsection we take $m=\omega_{0}=1$. In $3 \mathrm{D}$ the EPI matrix element is well known, $\left|V_{\mathbf{q}}\right|^{2}=2 \sqrt{2} \pi \alpha / V_{3} q^{2}$. The interaction coefficient in $n$ dimensions becomes (Peeters et al., 1986)

$$
\left|V_{\mathbf{q}}\right|^{2}=\frac{2^{n-3 / 2} \pi^{(n-1) / 2} \Gamma\left(\frac{n-1}{2}\right) \alpha}{V_{n} q^{n-1}}
$$

with $V_{n}$ the volume of the $n$-dimensional crystal.

The only difference between the model system in $n$ dimensions and the model system in $3 \mathrm{D}$ is that now one deals with an $n$-dimensional harmonic oscillator. Directly following (Feynman, 1955), the variational polaron energy was calculated in Ref. (Peeters et al., 1986)

$$
\begin{gathered}
E=\frac{n(v-w)}{2}-\frac{n\left(v^{2}-w^{2}\right)}{4 v}-\frac{2^{-3 / 2} \Gamma\left(\frac{n-1}{2}\right) \alpha}{\Gamma\left(\frac{n}{2}\right)} \int_{0}^{\infty} \frac{e^{-t}}{\sqrt{D_{0}(t)}} d t \\
=\frac{n(v-w)^{2}}{4 v}-\frac{\Gamma\left(\frac{n-1}{2}\right) \alpha}{2 \sqrt{2} \Gamma\left(\frac{n}{2}\right)} \int_{0}^{\infty} \frac{e^{-t}}{\sqrt{D_{0}(t)}} d t
\end{gathered}
$$

where

$$
D_{0}(t)=\frac{w^{2}}{2 v^{2}} t+\frac{v^{2}-w^{2}}{2 v^{3}}\left(1-e^{-v t}\right)
$$

In order to facilitate a comparison of $E$ for $n$ dimensions with the Feynman result (Feynman, 1955) for 3D,

$$
E_{3 \mathrm{D}}(\alpha)=\frac{3(v-w)^{2}}{4 v}-\frac{1}{\sqrt{2 \pi}} \alpha \int_{0}^{\infty} \frac{e^{-t}}{\sqrt{D_{0}(t)}} d t,
$$

it is convenient to rewrite Eq. (122) in the form

$$
E_{n \mathrm{D}}(\alpha)=\frac{n}{3}\left[\frac{3(v-w)^{2}}{4 v}-\frac{1}{\sqrt{2 \pi}} \frac{3 \sqrt{\pi} \Gamma\left(\frac{n-1}{2}\right)}{2 n \Gamma\left(\frac{n}{2}\right)} \alpha \int_{0}^{\infty} \frac{e^{-t}}{\sqrt{D_{0}(t)}} d t\right]
$$

The parameters $w$ and $v$ must be determined by minimizing $E$. In the case of Eq. (125) one should minimise the expression in the square brackets. The only difference of this expression 
from the rhs of Eq. (124) is that $\alpha$ is multiplied by the factor

$$
a_{n}=\frac{3 \sqrt{\pi} \Gamma\left(\frac{n-1}{2}\right)}{2 n \Gamma\left(\frac{n}{2}\right)} .
$$

This means that the minimizing parameters $w$ and $v$ in $n \mathrm{D}$ at a given $\alpha$ will be exactly the same as those calculated in 3D with the Fröhlich constant chosen as $a_{n} \alpha$ :

$$
v_{n \mathrm{D}}(\alpha)=v_{3 \mathrm{D}}\left(a_{n} \alpha\right), w_{n \mathrm{D}}(\alpha)=w_{3 \mathrm{D}}\left(a_{n} \alpha\right) .
$$

Comparing Eq. (125) to Eq. (124), the following scaling relation (Peeters and Devreese, 1987; Peeters et al., 1986; Xiaoguang et al., 1985) is obtained:

$$
E_{n \mathrm{D}}(\alpha)=\frac{n}{3} E_{3 \mathrm{D}}\left(a_{n} \alpha\right),
$$

where $a_{n}$ is given by Eq. (126). As discussed in Ref. (Peeters et al., 1986), the above scaling relation is not an exact relation. It is valid for the Feynman polaron energy and also for the ground-state energy to order $\alpha$. The next-order term (i.e., $\alpha^{2}$ ) e. g. no longer satisfies Eq. (128). The reason is that in the exact calculation (to order $\alpha^{2}$ ) the electron motions in different space directions are coupled by EPI. No such coupling appears in the Feynman polaron model; this is the underlying reason for the validity of the scaling relation for the Feynman approximation.

In Refs. (Devreese et al. , 1977; Peeters and Devreese, 1983a; Peeters and Devreese, 1987; Peeters et al., 1986), scaling relations were obtained also for the impedance function, $Z_{n \mathrm{D}}(\alpha ; \Omega)=Z_{3 \mathrm{D}}\left(a_{n} \alpha ; \Omega\right)$, the effective mass and the mobility of a polaron. In the important particular case of 2D, the scaling relations take the form (Peeters and Devreese, 1987; Peeters et al., 1986; Xiaoguang et al., 1985):

$$
\begin{aligned}
E_{2 \mathrm{D}}(\alpha) & =\frac{2}{3} E_{3 \mathrm{D}}\left(\frac{3 \pi}{4} \alpha\right), \\
Z_{2 \mathrm{D}}(\alpha ; \Omega) & =Z_{3 \mathrm{D}}\left(\frac{3 \pi}{4} \alpha ; \Omega\right), \\
\frac{m_{2 \mathrm{D}}^{*}(\alpha)}{(m)_{n \mathrm{D}}} & =\frac{m_{3 \mathrm{D}}^{*}\left(\frac{3 \pi}{4} \alpha\right)}{(m)_{3 \mathrm{D}}}, \\
\mu_{2 \mathrm{D}}(\alpha) & =\mu_{3 \mathrm{D}}\left(\frac{3 \pi}{4} \alpha\right) .
\end{aligned}
$$

The scaling relations (Peeters and Devreese, 1987) can be checked for the path integral Monte Carlo results (Titantah et al., 2001) for the polaron free energy given in 3D and in 2D for a few values of temperature and for some selected values of $\alpha$. They follow very closely the scaling relation of the form given by Eq.(129) (Devreese, 2006a). 


\section{DISCRETE HOLSTEIN AND FRÖHLICH POLARON}

When the coupling with phonons increases the polaron radius decreases and becomes of the order of the lattice constant. Then all momenta of the Brillouin zone contribute to the polaron wave function and the effective mass approximation cannot be applied. This regime occurs if the characteristic potential energy $E_{p}$ due to the local lattice deformation is compared or larger than the half-bandwidth $D$. The strong coupling regime with the dimensionless coupling constant

$$
\lambda \equiv \frac{E_{p}}{D} \geq 1
$$

is called the small or discrete lattice polaron. In general, $E_{p}$ is expressed as

$$
E_{p}=\frac{1}{2 N} \sum_{\mathbf{q}}|\gamma(\mathbf{q})|^{2} \omega_{\mathbf{q}}
$$

for any type of phonons involved in the polaron cloud. For the Fröhlich interaction with optical phonons one obtains $E_{p} \simeq q_{d} e^{2} / \pi \kappa$, where $q_{d}$ is the Debye momentum (Alexandrov, 1996). For example, with parameters appropriate for copper oxides $\varepsilon_{0} \gg \varepsilon \simeq 5$ and $q_{D} \simeq$ $0.7 \AA^{-1}$ one obtains $E_{p} \simeq 0.6 \mathrm{eV}$ (Alexandrov and Bratkovsky, 2000; Eagles, 1966). The exact value of $\lambda_{c}$ when the continuum (large) polaron transforms into the small one, depends on the lattice structure, phonon frequency dispersions and the radius of the electron-phonon interaction, but in most cases the transformation occurs around $\lambda_{c} \simeq 1$ (Alexandrov, 2001a).

\section{A. Holstein model}

For comparison we briefly introduce a small polaron created by a short-range EPI, which is known as the Holstein polaron. Its main features are revealed in the simple Holstein model (Holstein , 1959b) of two vibrating molecules and the electron hopping between them. A simplified version of the model is defined by a two-site Hamiltonian describing the electron tunnelling between sites 1 ("left") and 2 ("right") with the amplitude $t$ and interacting with a vibrational mode of an ion, placed at some distance in between, Fig 9 .

$$
H=t\left(c_{1}^{\dagger} c_{2}+c_{2}^{\dagger} c_{1}\right)+H_{p h}+H_{e-p h}
$$

Here we take the position of an atomic level in the rigid lattice as zero, and $c_{i}$ annihilates the electron on the left, $i=1$, or on the right, $i=2$, site. 

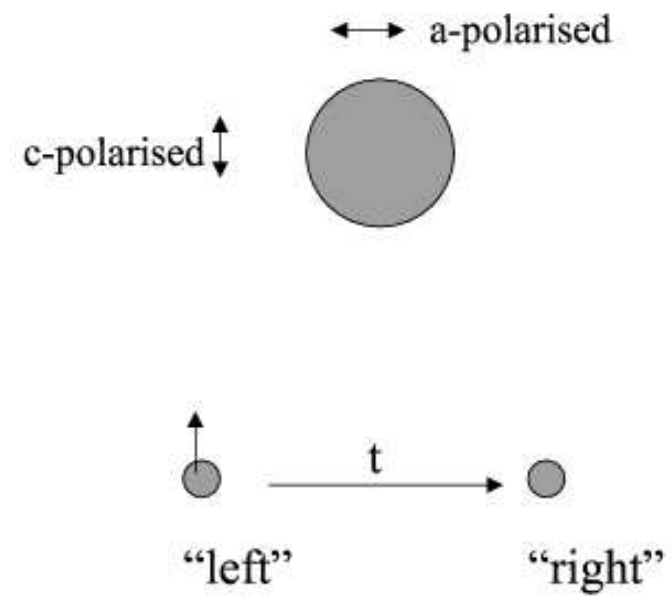

FIG. 9 Electron tunnels between sites 1 ("left") and 2 ("right") with the amplitude $t$ and interacts with c-axis or a-axis polarised vibrational modes of the ion, placed in between.

The vibration part of the Hamiltonian in this toy model is

$$
H_{p h}=-\frac{1}{2 M} \frac{\partial^{2}}{\partial x^{2}}+\frac{k x^{2}}{2}
$$

where $M$ is the ion mass, $k=M \omega_{0}^{2}$ is the spring constant, and $x$ is the ion displacement. The electron-phonon interaction, $H_{e-p h}$, depends on the polarization of vibrations. If the ion vibrates along the perpendicular direction to the hopping (in "c"-direction, Fig9) we have

$$
H_{e-p h}=f_{c} x\left(c_{1}^{\dagger} c_{1}+c_{2}^{\dagger} c_{2}\right)
$$

and

$$
H_{e-p h}=f_{a} x\left(c_{1}^{\dagger} c_{1}-c_{2}^{\dagger} c_{2}\right)
$$

if the ion vibrates along the hopping ("a" direction).

The wave-function of the electron and the ion is a linear superposition of two terms describing the electron on the "left" and on the "right" site, respectively,

$$
\psi=\left[u(x) c_{1}^{\dagger}+v(x) c_{2}^{\dagger}\right]|0\rangle
$$

where $|0\rangle$ is the vacuum state describing a rigid lattice without the extra electron. Substituting $\psi$ into the Schrödinger equation, $H \psi=E \psi$, we obtain two coupled equations for the amplitudes,

$$
\begin{aligned}
& \left(E-f_{a, c} x-H_{p h}\right) u(x)=t v(x), \\
& \left(E \pm f_{a, c} x-H_{p h}\right) v(x)=t u(x),
\end{aligned}
$$


where + and - in the second equation correspond to "a" and "c" polarised vibrations, respectively. There is the exact solution for the "c" -axis polarization, when a change in the ion position leads to the same shift of the electron energy on the left and on the right sites,

$$
\begin{aligned}
& u(x)=u \chi_{n}(x), \\
& v(x)=v \chi_{n}(x),
\end{aligned}
$$

where $u$ and $v$ are constants and

$$
\chi_{n}(x)=\left(\frac{M \omega_{0}}{\pi\left(2^{n} n !\right)^{2}}\right)^{1 / 4} H_{n}\left[\left(x-f_{c} / k\right)\left(M \omega_{0}\right)^{1 / 2}\right] \exp \left[-M\left(x-f_{c} / k\right)^{2} \omega_{0} / 2\right],
$$

is the harmonic oscillator wave-function. There are two ladders of levels given by

$$
E_{n}^{ \pm}=-E_{p} \pm t+\omega_{0}(n+1 / 2)
$$

with $E_{p}=f_{c}^{2} / 2 k$. Here $H_{n}(\xi)$ are the Hermite polynomials, and $n=0,1,2,3, \ldots$. Hence the c-axis single-ion deformation leads to the polaron level shift but without any renormalisation of the hopping integral $t$. In contrast, $a$-polarized vibrations with the opposite shift of the electron energy on the left and on the right sites, strongly renormalise the hopping integral. There is no simple general solution of the Holstein model in this case, but one can find it in two limiting cases, non-adiabatic, when $t \ll \omega_{0}$ and adiabatic, when $t \gg \omega_{0}$.

\section{Non-adiabatic Holstein polaron}

In the non-adiabatic regime the ion vibrations are fast and the electron hopping is slow. Hence one can apply a perturbation theory in powers of $t$ to solve

$$
\left(\begin{array}{cc}
E-f_{a} x-H_{p h} & -t \\
-t & E+f_{a} x-H_{p h}
\end{array}\right)\left[\begin{array}{l}
u(x) \\
v(x)
\end{array}\right]=0
$$

One takes $t=0$ in zero order, and obtains a two-fold degenerate ground state $\left[u^{l, r}(x), v^{l, r}(x)\right]$, corresponding to the polaron localised on the left $(l)$ or on the right $(r)$ sites,

$$
\begin{array}{r}
u^{l}(x)=\exp \left[-\frac{M \omega_{0}}{2}\left(x+f_{a} / k\right)^{2}\right] \\
v^{l}(x)=0
\end{array}
$$

and

$$
u^{r}(x)=0
$$




$$
v^{r}(x)=\exp \left[-\frac{M \omega_{0}}{2}\left(x-f_{a} / k\right)^{2}\right]
$$

with the energy $E_{0}=-E_{p}+\omega_{0} / 2$, where $E_{p}=f_{a}^{2} / 2 k$. The eigenstates are found as linear superpositions of two unperturbed states,

$$
\left[\begin{array}{l}
u(x) \\
v(x)
\end{array}\right]=\alpha\left[\begin{array}{c}
u^{l}(x) \\
0
\end{array}\right]+\beta\left[\begin{array}{c}
0 \\
v^{r}(x)
\end{array}\right] .
$$

Here the coefficients $\alpha$ and $\beta$ are independent of $x$. The conventional secular equation for $E$ is obtained, multiplying the first row by $u^{l}(x)$ and the second row by $v^{r}(x)$, and integrating over the vibration coordinate, $x$, each of two equations of the system. The result is

$$
\operatorname{det}\left(\begin{array}{cc}
E-E_{0} & \tilde{t} \\
\tilde{t} & E-E_{0}
\end{array}\right)=0
$$

with the renormalised hopping integral

$$
\frac{\tilde{t}}{t}=\frac{\int_{-\infty}^{\infty} d x u^{l}(x) v^{r}(x)}{\int_{-\infty}^{\infty} d x\left|u^{l}(x)\right|^{2}} .
$$

The corresponding eigenvalues, $E_{ \pm}$are

$$
E_{ \pm}=\omega_{0} / 2-E_{p} \pm \tilde{t}
$$

The hopping integral splits the degenerate level, as in the rigid lattice, but an effective 'bandwidth' $2 \tilde{t}$ is significantly reduced compared with the bare one,

$$
\tilde{t}=t \exp \left(-2 E_{p} / \omega_{0}\right)
$$

This polaron band narrowing originates in a small overlap integral of two displaced oscillator wave functions $u^{l}(x)$ and $v^{r}(x)$.

\section{Adiabatic Holstein polaron}

In the adiabatic regime, when $t \gg \omega_{0}$, the electron tunnelling is fast compared with the ion motion. Hence one can apply the Born-Oppenheimer adiabatic approximation (Born and Oppenheimer, 1927) taking the wave function in the form

$$
\left[\begin{array}{l}
u(x) \\
v(x)
\end{array}\right]=\chi(x)\left[\begin{array}{l}
u_{a}(x) \\
v_{a}(x)
\end{array}\right] .
$$


Here $u_{a}(x)$ and $v_{a}(x)$ are the electron wave functions obeying the Schrödinger equation with the frozen ion deformation $x$, i.e.

$$
\left(\begin{array}{cc}
E_{a}(x)-f_{a} x & -t \\
-t & E_{a}(x)+f_{a} x
\end{array}\right)\left[\begin{array}{l}
u_{a}(x) \\
v_{a}(x)
\end{array}\right]=0 .
$$

The lowest energy level is found as

$$
E_{a}(x)=-\sqrt{\left(f_{a} x\right)^{2}+t^{2}} .
$$

$E_{a}(x)$ together with $k x^{2} / 2$ plays the role of a potential energy term in the equation for the 'vibration' wave function, $\chi(x)$,

$$
\left[-\frac{1}{2 M} \frac{\partial^{2}}{\partial x^{2}}+\frac{k x^{2}}{2}-\sqrt{\left(f_{a} x\right)^{2}+t^{2}}\right] \chi(x)=E \chi(x) .
$$

Terms with the first and second derivatives of the electron wave-functions $u_{a}(x)$ and $v_{a}(x)$ are small compared with the corresponding derivatives of $\chi(x)$ in the adiabatic approximation, so they are neglected in Eq.(157). As a result we arrive at the familiar double-well potential problem, where the potential energy $U(x)=k x^{2} / 2-\sqrt{\left(f_{a} x\right)^{2}+t^{2}}$ has two symmetric minima, separated by a barrier. Minima are located approximately at

$$
x_{m}= \pm f_{a} / k
$$

in the strong-coupling limit, $E_{p} \gg t$, and the potential energy near the bottom of each potential well is about

$$
U(x)=-E_{p}+\frac{k\left(|x|-f_{a} / k\right)^{2}}{2} .
$$

If the barrier were impenetrable, there would be the ground state energy level $E_{0}=-E_{p}+$ $\omega_{0} / 2$, the same for both wells. The underbarrier tunnelling results in a splitting of this level $2 \tilde{t}$, which corresponds to a polaron bandwidth in the lattice. It can be estimated using the quasi-classical approximation as

$$
\tilde{t} \propto \exp \left[-2 \int_{0}^{x_{m}} p(x) d x\right]
$$

where $p(x)=\sqrt{2 M\left[U(x)-E_{0}\right]} \approx(M k)^{1 / 2}\left|x-f_{a} / k\right|$ is the classical momentum

Estimating the integral one finds the exponential reduction of the "bandwidth",

$$
\tilde{t} \propto \exp \left(-2 E_{p} / \omega_{0}\right)
$$


which is the same as in the non-adiabatic regime. Holstein found corrections to this expression up to terms of the order of $1 / \lambda^{2}$, which allowed him to improve the exponent and estimate the pre-exponential factor as

$$
\tilde{t} \approx \sqrt{\frac{E_{p} \omega_{0}}{\pi}} e^{-\tilde{g}^{2}} .
$$

Here $g^{2}=2 E_{p} / \omega_{0}$ and

$$
\tilde{g}^{2}=g^{2}\left[1-\frac{1}{4 \lambda^{2}} \ln (4 \lambda)-\frac{1}{8 \lambda^{2}}\right] .
$$

A more accurate expression for $\tilde{t}$ was obtained in (Alexandrov et al. , 1994a),

$$
\tilde{t} \approx \sqrt{\frac{E_{p} \omega_{0}}{\pi}} \beta^{5 / 2} \lambda^{1-\beta}[2(1+\beta)]^{-\beta} e^{-\tilde{g}^{2}},
$$

where now $\tilde{g}^{2}=g^{2}\left\{\beta-[\ln (2 \lambda(1+\beta))] / 4 \lambda^{2}\right\}$, and $\lambda=E_{p} / t$. This expression takes into account the phonon frequency renormalisation, $\beta \equiv \tilde{\omega}_{0} / \omega_{0}=\sqrt{1-1 / 4 \lambda^{2}}$, and the anharmonic corrections of the order of $1 / \lambda^{2}$ to the turning point $x_{m}$ in Eq.(160). The term in front of the exponent in Eqs. (162,164) differs from $t$ of the non-adiabatic case, Eq.(153). It is thus apparent that the perturbation approach covers only a part of the entire lattice polaron region, $\lambda \gtrsim 1$. The upper limit of applicability of the perturbation theory is given by $t<\sqrt{E_{p} \omega_{0}}$. For the remainder of the region the adiabatic approximation is more appropriate. A much lower effective mass of the adiabatic small polaron in the intermediate coupling region compared with that estimated from the perturbation theory expression, Eq.(153), is revealed in Eq.(164) (Alexandrov et al. , 1994a). The double well potential disappears at $\lambda=\lambda_{c}=0.5$, where the renormalised phonon frequency $\tilde{\omega}_{0}$ is zero.

The Holstein polaron model can be readily generalized for infinite lattices (section IV.B). Similar models were used, for instance, in studies of dissipation (Caldeira and Leggett), 1981; Castro Neto and Caldeira , 1991) and effects of decoherence in open quantum-mechanical systems.

\section{B. Lang-Firsov canonical transformation}

The kinetic energy is smaller than the interaction energy as long as $\lambda>1$. Hence a self-consistent approach to the discrete (or lattice) polaron problem with EPI of any range is possible with the " $1 / \lambda$ " expansion technique (Lang and Firsov, 1962, 1963) on infinite lattices with any type of EPI conserving the electron site-occupation numbers, and any 
phonon spectrum. The technique treats the electron kinetic energy as a perturbation, and can be applied for multi-polaron systems as well (Alexandrov, 1983) (see VI.B). It is based on the fact, known for a long time, that there is an analytical exact solution of the any - number polaron problem in the extreme strong-coupling limit, $\lambda \rightarrow \infty$. Following Lang and Firsov one applies the canonical transformation $e^{S}$ to diagonalise the Hamiltonian, Eq.(11). The diagonalisation is exact, if $t(\mathbf{m})=0$ (or $\lambda=\infty)$ :

$$
\tilde{H}=e^{S} H e^{-S}
$$

where

$$
S=-\sum_{\mathbf{q}, i} \hat{n}_{i}\left[u_{i}(\mathbf{q}) d_{\mathbf{q}}-H . c .\right]
$$

is such that $S^{\dagger}=-S$. The electron and phonon operators are transformed as $\tilde{c}_{i}=e^{S} c_{i} e^{-S}$ and $\tilde{d}_{\mathbf{q}}=e^{S} d_{\mathbf{q}} e^{-S}$. The result is

$$
\tilde{c}_{i}=c_{i} \hat{X}_{i}
$$

and

$$
\tilde{d}_{\mathbf{q}}=d_{\mathbf{q}}-\sum_{i} \hat{n}_{i} u_{i}^{*}(\mathbf{q})
$$

where $\hat{X}_{i}=\exp \left[\sum_{\mathbf{q}} u_{i}(\mathbf{q}) d_{\mathbf{q}}-\right.$ H.c. $]$. The Lang-Firsov transformation shifts the ions to new equilibrium positions. In a more general sense it changes the boson vacuum. As a result, the transformed Hamiltonian takes the following form

$$
\tilde{H}=\sum_{i, j} \hat{\sigma}_{i j} c_{i}^{\dagger} c_{j}-E_{p} \sum_{i} \hat{n}_{i}+\sum_{\mathbf{q}} \omega_{\mathbf{q}}\left(d_{\mathbf{q}}^{\dagger} d_{\mathbf{q}}+1 / 2\right)+\frac{1}{2} \sum_{i \neq j} v_{i j} \hat{n}_{i} \hat{n}_{j},
$$

where

$$
\hat{\sigma}_{i j}=t(\mathbf{m}-\mathbf{n}) \delta_{s s^{\prime}} \hat{X}_{i}^{\dagger} \hat{X}_{j}
$$

is the renormalised hopping integral depending on the phonon operators, and

$$
v_{i j}=V_{c}(\mathbf{m}-\mathbf{n})-\frac{1}{N} \sum_{\mathbf{q}}|\gamma(\mathbf{q})|^{2} \omega_{\mathbf{q}} \cos [\mathbf{q} \cdot(\mathbf{m}-\mathbf{n})]
$$

is the interaction of polarons, $v_{i j} \equiv v(\mathbf{m}-\mathbf{n})$, comprising their Coulomb repulsion and the interaction via the lattice deformation. In the extreme infinite-coupling limit, $\lambda \rightarrow \infty$, we can neglect the hopping term of the transformed Hamiltonian. The rest has analytically 
determined eigenstates and eigenvalues. The eigenstates $|\tilde{N}\rangle=\left|n_{i}, n_{\mathbf{q}}\right\rangle$ are sorted by the polaron $n_{\mathbf{m} s}$ and phonon $n_{\mathbf{q}}$ occupation numbers. The energy levels are

$$
E=-\left(\mu+E_{p}\right) \sum_{i} n_{i}+\frac{1}{2} \sum_{i \neq j} v_{i j} n_{i} n_{j}+\sum_{\mathbf{q}} \omega_{\mathbf{q}}\left(n_{\mathbf{q}}+1 / 2\right),
$$

where $n_{i}=0,1$ and $n_{\mathbf{q}}=0,1,2,3, \ldots \infty$.

\section{1. " $1 / \lambda "$ expansion and polaron band}

The Hamiltonian $\tilde{H}$ in zero order with respect to the hopping describes localised polarons and independent phonons, which are vibrations of ions relative to new equilibrium positions depending on the polaron occupation numbers. The middle of the electron band is shifted down by the polaron level-shift $E_{p}$ due to the potential well created by lattice deformation. Importantly the phonon frequencies remain unchanged in this limit at any polaron density, $n$. At finite $\lambda$ and $n$ there is a softening of phonons $\delta \omega_{0}$ of the order of $\omega_{0} n / \lambda^{2}$ (Alexandrov, 1992a; Alexandrov and Capellmann, 1991; Alexandrov et al. , 1992; Göbel et al., 1994) (the initial paper on the phonon renormalisation (Alexandrov and Capellmann , 1991) predicting $\delta \omega_{0} \propto 1 / \lambda$ was subsequently corrected (Alexandrov, 1992a; Alexandrov et al. , 1992; Göbel et al., 1994)). Interestingly the optical phonon can be mixed with a low-frequency polaronic plasmon forming a new excitation, "plasphon", which was proposed in (Alexandrov, 1992a) as an explanation of the anomalous phonon mode splitting observed in cuprates (Rietschel et al. , 1989).

Now let us discuss the $1 / \lambda$ expansion. First we restrict the discussion to a single-polaron problem with no polaron-polaron interaction. The finite hopping term leads to the polaron tunnelling because of degeneracy of the zero order Hamiltonian with respect to the site

position of the polaron. To see how the tunnelling occurs we apply the perturbation theory using $1 / \lambda$ as a small parameter. The proper Bloch set of $N$-fold degenerate zero order eigenstates with the lowest energy $\left(-E_{p}\right)$ of the unperturbed Hamiltonian is

$$
|\mathbf{k}, 0\rangle=\frac{1}{\sqrt{N}} \sum_{\mathbf{m}} c_{\mathbf{m} s}^{\dagger} \exp (i \mathbf{k} \cdot \mathbf{m})|0\rangle
$$

where $|0\rangle$ is the vacuum, and $N$ is the number of sites. By applying the textbook perturbation theory one readily calculates the perturbed energy levels. Up to the second order in 
the hopping integral they are given by

$$
E_{\mathbf{k}}=-E_{p}+\epsilon_{\mathbf{k}}-\sum_{\mathbf{k}^{\prime}, n_{\mathbf{q}}} \frac{\left|\left\langle\mathbf{k}, 0\left|\sum_{i, j} \hat{\sigma}_{i j} c_{i}^{\dagger} c_{j}\right| \mathbf{k}^{\prime}, n_{\mathbf{q}}\right\rangle\right|^{2}}{\sum_{\mathbf{q}} \omega_{\mathbf{q}} n_{\mathbf{q}}},
$$

where $\left|\mathbf{k}^{\prime}, n_{\mathbf{q}}\right\rangle$ are the exited states of the unperturbed Hamiltonian with one electron and at least one real phonon. The second term in this equation, which is linear with respect to the bare hopping $t(\mathbf{m})$, describes the polaron-band dispersion (Alexandrov, 2000),

$$
\epsilon_{\mathbf{k}}=\sum_{\mathbf{m}} t(\mathbf{m}) e^{-g^{2}(\mathbf{m})} \exp (-i \mathbf{k} \cdot \mathbf{m})
$$

where

$$
g^{2}(\mathbf{m})=\frac{1}{2 N} \sum_{\mathbf{q}}|\gamma(\mathbf{q})|^{2}[1-\cos (\mathbf{q} \cdot \mathbf{m})]
$$

is the band-narrowing factor at zero temperature.

The third term, quadratic in $t(\mathbf{m})$, yields a negative $\mathbf{k}$-independent correction to the polaron level-shift of the order of $1 / \lambda^{2}$, and a small correction to the polaron band dispersion, Eq.(175) (Alexandrov, 1992a; Gogolin, 1982; Kudinov and Firsov, 1997). The correction to the level shift is due to polaronic hops onto a neighbouring site with no deformation around it. As any second order correction this transition shifts the energy down by an amount of about $-t^{2}(\mathbf{m}) / E_{p}$. It has little to do with the polaron effective mass and the polaron tunneling mobility because the lattice deformation does not follow the electron. The polaron hops back and forth many times (about $e^{g^{2}}$ ) "waiting" for a sufficient lattice deformation to appear around neighbouring site $\mathbf{n}$. Only after the deformation around the neighbouring site is created does the polaron tunnel onto the next site together with the deformation.

\section{Temperature effect on the polaron band}

Let us now analyse the temperature dependence of the polaron bandwidth, which is determined by the average of the multiphonon operator, Eq.(170),

$$
\left\langle\left\langle\hat{X}_{i}^{\dagger} \hat{X}_{j}\right\rangle\right\rangle \equiv \prod_{\mathbf{q}}\left\langle\left\langle\exp \left[u_{i}^{*}(\mathbf{q}) d_{\mathbf{q}}^{\dagger}-H . c .\right] \exp \left[u_{j}(\mathbf{q}) d_{\mathbf{q} \nu}-H . c .\right]\right\rangle\right\rangle .
$$

Here the double angular brackets correspond to quantum as well as statistical averages of any operator $\hat{A}$ with the Gibbs distribution,

$$
\langle\langle\hat{A}\rangle\rangle=\sum_{\nu} e^{\left(\Omega-E_{\nu}\right) / T}\langle\nu|\hat{A}| \nu\rangle \equiv \operatorname{Tr}\left\{e^{(\Omega-\tilde{H}) / T} \hat{A}\right\},
$$


where $\Omega$ is the thermodynamic potential and $|\nu\rangle$ are the eigenstates of $\tilde{H}$ with the eigenvalues $E_{\nu}$. An operator identity $\exp (\hat{A}+\hat{B})=\exp (\hat{A} \exp (\hat{B}) \exp (-[\hat{A}, \hat{B}] / 2)$ is instrumental. It is applied when the commutator $[\hat{A}, \hat{B}]$ is a number. The identity allows us to write

$$
\begin{aligned}
e^{\left[u_{i}^{*}(\mathbf{q}) d_{\mathbf{q}}^{\dagger}-H . c .\right]} e^{\left[u_{j}(\mathbf{q}) d_{\mathbf{q}}-H . c .\right]}= & e^{\alpha^{*} d_{\mathbf{q}}^{\dagger}} e^{-\alpha d_{\mathbf{q}}} e^{-|\alpha|^{2} / 2} \times \\
& e^{\left[u_{i}(\mathbf{q}) u_{j}^{*}(\mathbf{q})-u_{i}^{*}(\mathbf{q}) u_{j}(\mathbf{q})\right] / 2} .
\end{aligned}
$$

Quantum and statistical averages are calculated by expanding the exponents in the trace as

$$
\left\langle\left\langle e^{\alpha^{*} d^{\dagger}} e^{-\alpha d}\right\rangle\right\rangle=(1-p) \sum_{N=0}^{\infty} \sum_{n=0}^{N} p^{N}(-1)^{n} \frac{|\alpha|^{2 n}}{(n !)^{2}} N(N-1) \times \ldots \times(N-n+1),
$$

where we dropped the phonon and site quantum numbers for transparency. Here $p=$ $\exp \left(-\omega_{\mathbf{q} \nu} / T\right)$, so that a single-mode phonon partition function is $Z_{p h}=1 /(1-p)$. Eq. (180) can be written in the form (Lang and Firsov, 1962)

$$
\left\langle\left\langle e^{\alpha^{*} d^{\dagger}} e^{-\alpha d}\right\rangle\right\rangle=(1-p) \sum_{n=0}^{N}(-1)^{n} \frac{|\alpha|^{2 n}}{(n !)^{2}} p^{n} \frac{d^{n}}{d p^{n}} \sum_{N=0}^{\infty} p^{N}
$$

Taking the sum over $N, \sum_{N=0}^{\infty} p^{N}=1 /(1-p)$, and differentiating it $n$ times yield $n$ ! in the numerator, after which the series over $n$ turns out equal to

$$
\left\langle\left\langle e^{\alpha^{*} d^{\dagger}} e^{-\alpha d}\right\rangle\right\rangle=e^{-|\alpha|^{2} n_{\omega}}
$$

where $n_{\omega}=\left[\exp \left(\omega_{\mathbf{q}} / T\right)-1\right]^{-1}$ is the Bose-Einstein phonon distribution function. Collecting all multipliers one finally obtains

$$
\left\langle\left\langle\hat{\sigma}_{i j}\right\rangle\right\rangle=T(\mathbf{m}-\mathbf{n}) \delta_{s s^{\prime}} \exp \left(-\frac{1}{2 N} \sum_{\mathbf{q}}|\gamma(\mathbf{q})|^{2}[1-\cos (\mathbf{q} \cdot \mathbf{m})] \operatorname{coth} \frac{\omega_{\mathbf{q}}}{2 T}\right),
$$

with the zero-temperature limit given by Eq.(175).

The small-polaron band is exponentially narrow. Hence, one can raise a concern about its existence in real solids (De Mello and Ranninger, 1998a). At zero temperature the perturbation term of the transformed Hamiltonian conserves the momentum because all off-diagonal matrix elements vanish,

$$
\left\langle\mathbf{k}, 0\left|\sum_{i, j} \hat{\sigma}_{i, j} c_{i}^{\dagger} c_{j}\right| \mathbf{k}^{\prime}, 0\right\rangle=0
$$


if $\mathbf{k} \neq \mathbf{k}^{\prime}$. The emission of a single high-frequency phonon is impossible for any $\mathbf{k}$ because of the energy conservation. The polaron half-bandwidth is exponentially reduced,

$$
w \approx D e^{-g^{2}}
$$

and it is usually less than the optical phonon energy $\omega_{0}\left(g^{2}\right.$ is about $\left.D \lambda / \omega_{0}\right)$. Hence, there is no damping of the polaron band at $T=0$ caused by optical phonons, no matter how strong the interaction is. The phonons "dress" the electron and coherently follow its motion. However, at finite temperatures the simultaneous emission and absorption of phonons is possible. Moreover the polaron bandwidth shrinks with increasing temperature because the phonon-averaged hopping integrals depend on temperature, Eq.(183). For high temperatures, $T \gg \omega_{0} / 2$, the band narrows exponentially as $w \approx D e^{-T / T_{0}}$, where

$$
T_{0}^{-1}=\frac{1}{N} \sum_{\mathbf{q}}|\gamma(\mathbf{q})|^{2} \omega_{\mathbf{q}}^{-1}[1-\cos (\mathbf{q} \cdot \mathbf{m})] .
$$

On the other hand, the two-phonon scattering of polarons becomes more important with increasing temperature. One can estimate this scattering rate by applying the Fermi-Dirac golden rule,

$$
\frac{1}{\tau}=2 \pi\left\langle\sum_{\mathbf{q}, \mathbf{q}^{\prime}}\left|M_{\mathbf{q} \mathbf{q}^{\prime}}\right|^{2} \delta\left(\epsilon_{\mathbf{k}}-\epsilon_{\mathbf{k}+\mathbf{q}-\mathbf{q}^{\prime}}\right)\right\rangle,
$$

where the corresponding matrix element is

$$
M_{\mathbf{q q}^{\prime}}=\sum_{i, j}\left\langle\mathbf{k}+\mathbf{q}-\mathbf{q}^{\prime}, n_{\mathbf{q}}-1, n_{\mathbf{q}^{\prime}}+1\left|\hat{\sigma}_{i, j} c_{i}^{\dagger} c_{j}\right| \mathbf{k}, n_{\mathbf{q}}, n_{\mathbf{q}^{\prime}}\right\rangle .
$$

For simplicity we consider the momentum independent $\gamma(\mathbf{q})=\gamma_{0}$ and $\omega_{\mathbf{q}}=\omega_{0}$. Expanding $\hat{\sigma}_{i j}$-operators in powers of the phonon creation and annihilation operators one estimates the matrix element of the two-phonon scattering as $M_{\mathbf{q q}^{\prime}} \approx N^{-1} w \gamma_{0}^{2} \sqrt{n_{\mathbf{q}}\left(n_{\mathbf{q}^{\prime}}+1\right)}$. Using this estimate and the polaron density of states (DOS), $\rho_{p}(\xi) \equiv N^{-1} \sum_{\mathbf{k}} \delta\left(\xi-\epsilon_{\mathbf{k}}\right) \approx 1 / 2 w$ one obtains (Alexandrov and Mott, 1995)

$$
\frac{1}{\tau} \approx w \gamma_{0}^{4} n_{\omega}\left(1+n_{\omega}\right)
$$

where $n_{\omega}=\left[\exp \left(\omega_{0} / T\right)-1\right]^{-1}$ is the phonon distribution function.

The polaron band is well defined, if $1 / \tau<w$, which is satisfied for a temperature range $T \leq T_{\min } \approx \omega_{0} / \ln \gamma_{0}^{4}$ about half of the characteristic phonon frequency for relevant values of $\gamma_{0}^{2}$. At higher temperatures the incoherent thermal activated hopping dominates in the polaron dynamics (Holstein, 1959b; Lang and Firsov, 1962; Sewell, 1958; 
Yamashita and Kurosawa, 1958), and the polaron states are no longer the Bloch states. When the optical phonon frequencies are exceptionally high (i.e. about $1000 \mathrm{~K}$ as in hightemperature superconductors (Rietschel et al. , 1989)) lattice polarons are in the Bloch states in the relevant range of temperatures, where the Boltzmann kinetic theory with renormalised energy spectrum is applied.

\section{Discrete Fröhlich polaron at strong coupling}

The narrowing of the band and the polaron effective mass strongly depend on the range of EPI (Alexandrov, 1996; Eagles , 1969). Let us compare the small Holstein polaron (SHP) formed by the zero-range EPI and a small polaron formed by the long-range (Fröhlich) interaction, which we refer to as the small or discrete Fröhlich polaron (SFP). We use the real-space representation of $H_{e-p h}$ (Alexandrov and Kornilovitch, 1999),

$$
H_{e-p h}=\sum_{\mathbf{n}, i} f(\mathbf{m}-\mathbf{n}) \xi_{\mathbf{n}} \hat{n}_{i}
$$

with the normal coordinate at site $\mathbf{n}$

$$
\xi_{\mathbf{n}}=\sum_{\mathbf{q}}\left(2 N M \omega_{\mathbf{q}}\right)^{-1 / 2} e^{i \mathbf{q} \cdot \mathbf{n}} d_{\mathbf{q}}+H . c .
$$

and the force between the electron at site $\mathbf{m}$ and the normal coordinate $\xi_{\mathbf{n}}$,

$$
f(\mathbf{m})=N^{-1} \sum_{\mathbf{q}} \gamma(\mathbf{q})\left(M \omega_{\mathbf{q}}^{3}\right)^{1 / 2} e^{i \mathbf{q} \cdot \mathbf{m}} .
$$

In general, there is no simple relation between the polaron level-shift $E_{p}$ and the exponent $g^{2}$ of the mass enhancement. This relation depends on the form of EPI. Indeed for EPI with a single dispersionless phonon mode, $\omega_{\mathbf{q}}=\omega_{0}$, one obtains

$$
E_{p}=\frac{1}{2 M \omega_{0}^{2}} \sum_{\mathbf{m}} f^{2}(\mathbf{m}),
$$

and

$$
g^{2}=\frac{1}{2 M \omega_{0}^{3}} \sum_{\mathbf{m}}\left[f^{2}(\mathbf{m})-f(\mathbf{m}) f(\mathbf{m}+\mathbf{a})\right],
$$

where $\mathbf{a}$ is the primitive lattice vector. In the nearest-neighbour approximation the effective mass renormalisation is given by $m^{*} / m=e^{g^{2}}$ where $1 / m^{*}=\partial^{2} \epsilon_{\mathbf{k}} / \partial k^{2}$ at $k \rightarrow 0$ is the inverse polaron mass. If the interaction is short-ranged, $f(\mathbf{m})=\kappa \delta_{\mathbf{m}, 0}$ (the Holstein model), 


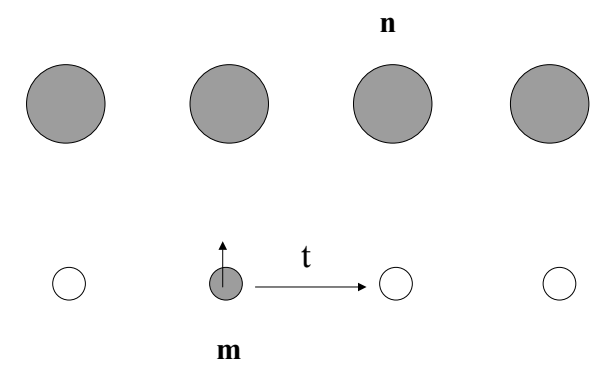

FIG. 10 A one-dimensional model of the lattice polaron on chain $\mathbf{m}$ interacting with displacements of all ions of another chain $\mathbf{n}$ ( $t$ is the polaron hopping integral along chain $\mathbf{m})$.

then $g^{2}=E_{p} / \omega_{0}$. Here $\kappa$ is a constant. In general, one has $g^{2}=\gamma E_{p} / \omega_{0}$ with the numerical coefficient

$$
\gamma=\frac{1-\sum_{\mathbf{m}} f(\mathbf{m}) f(\mathbf{m}+\mathbf{a})}{\sum_{\mathbf{n}} f^{2}(\mathbf{n})},
$$

which might be less than 1. To estimate $\gamma$ let us consider a one-dimensional chain model with the long-range Coulomb interaction between the electron on one chain $(\mathbf{m})$ and ion vibrations of another chain $(\mathbf{n})$, polarised in the direction perpendicular to the chains (Alexandrov and Kornilovitch, 1999), Fig[10. The corresponding force is given by

$$
f(\mathbf{m}-\mathbf{n})=\frac{\kappa}{\left(|\mathbf{m}-\mathbf{n}|^{2}+1\right)^{3 / 2}} .
$$

Here the distance along the chains $|\mathbf{m}-\mathbf{n}|$ is measured in units of the lattice constant $a$, the inter-chain distance is also $a$, and we take $a=1$. For this long-range interaction we obtain $E_{p}=1.27 \kappa^{2} /\left(2 M \omega_{0}^{2}\right), g^{2}=0.49 \kappa^{2} /\left(2 M \omega_{0}^{3}\right)$ and $g^{2}=0.39 E_{p} / \omega_{0}$. Thus the effective mass renormalisation in the non-adiabatic regime is much smaller than in the Holstein model, roughly as $m_{S F P}^{*} \propto\left(m_{S H P}^{*}\right)^{1 / 2}$ in units of $m$. An analytical solution of a two-site single electron system interacting with many vibrating ions of a lattice via a long-range Fröhlich EPI found that SFP is also several orders of magnitude lighter than SHP in the adiabatic regime (Alexandrov and Yavidov, 2004).

Another interesting point is that the size of SFP and the length, over which the distortion spreads, are different. In the strong-coupling limit the polaron is almost localised on one site $\mathbf{m}$. Hence, the size of its wave function is the atomic size. On the other hand, the 
ion displacements, proportional to the displacement force $f(\mathbf{m}-\mathbf{n})$, spread over a large distance. Their amplitude at a site $\mathbf{n}$ falls with the distance as $|\mathbf{m}-\mathbf{n}|^{-3}$ in the onedimensional model, Fig.10, The polaron cloud (i.e. lattice distortion) is more extended than the polaron itself. Such polaron tunnels with a larger probability than the Holstein polaron due to a smaller relative lattice distortion around two neighbouring sites. For a short-range EPI the entire lattice deformation disappears at one site and then forms at its neighbour, when the polaron tunnels from site to site. Therefore $\gamma=1$ and the polaron is very heavy already at $\lambda \approx 1$. On the contrary, if the interaction is long-ranged, only a fraction of the total deformation changes every time the polaron tunnels from one site to its neighbour, and $\gamma$ is smaller than 1.

\section{Effect of dispersive phonons}

A lighter mass of SFP compared with the nondispersive SHP is a generic feature of any dispersive electron-phonon interaction. For example, a short-range interaction with dispersive acoustic phonons $\left(\gamma(\mathbf{q}) \propto 1 / q^{1 / 2}, \omega_{\mathbf{q}} \propto q\right)$ also leads to a lighter polaron in the strong-coupling regime compared with SHP (Alexandrov, 2003). Even within the Holstein model with the local (intramolecular) EPI the dispersion of phonon frequencies is a vital ingredient since nondispersive phonons might lead to a divergent site jump probability of polarons (Yamashita and Kurosawa, 1958). Importantly the comprehensive studies of the molecular Holstein Hamiltonian, in which the dispersive features of the phonon spectrum are taken into account, found much lower values of the polaron mass compared with the non-dispersive model (Zoli , 1998, 2000; Zoli and Das , 2004). In those studies the $1 / \lambda$ perturbation theory based on the standard Lang-Firsov (LF) and the variational (modified) MLF transformation of the molecular Holstein Hamiltonian with dispersive phonons has been applied for 1D, 2D and 3D lattices including the second-order corrections as in Eq.(174),

$$
H=-t \sum_{\langle i, j\rangle} c_{i}^{\dagger} c_{j}+g \omega_{0} \sum_{i} \hat{n}_{i}\left(d_{i}+d_{i}^{\dagger}\right)+\sum_{\mathbf{q}} \omega_{\mathbf{q}}\left(d_{\mathbf{q}}^{\dagger} d_{\mathbf{q}}+1 / 2\right),
$$

where $d_{\mathbf{q}}$ is the Fourier transform of $d_{i}$. The phonon dispersion has been modeled using the intermolecular first neighbours force constant, $M \omega_{1}^{2}$, which yields, for example, in $1 \mathrm{D}$ case $\omega_{q}^{2}=\omega_{0}^{2} / 2+\omega_{1}^{2}+\left(\omega_{0}^{2} / 4+\omega_{0}^{2} \omega_{1}^{2} \cos q+\omega_{1}^{2}\right)^{1 / 2}$. MLF improves the convergence of the $1 / \lambda$ perturbation series by introducing a suitable variational parameter $\lambda_{\mathbf{q}}$ in the LF 
transformation, Eq.(166), as (Chatterjee and Das, 2000; Chatterjee et al. , 2003)

$$
S \Rightarrow S_{M L F}=\sum_{\mathbf{q}, \mathbf{k}} \lambda_{\mathbf{q}} c_{\mathbf{k}+\mathbf{q}}^{\dagger} c_{\mathbf{k}}\left(d_{\mathbf{q}}-d_{-\mathbf{q}}^{\dagger}\right)
$$

As a result the polaron mass converges to much lower values when the phonon dispersion is introduced, in particular in the adiabatic regime. There is a continuous mass enhancement whose abruptness is significantly smoothed for the largest values of the phonon dispersion, similar to the SFP discussed above. While a phase transition is ruled out in the single-electron Holstein Hamiltonian, where the ground state energy is analytic in the EPI strength (Gerlach and Löwen , 1991; Löwen , 1988), a crossover from more itinerant to more self-trapped behaviour may be identified as a rather sudden event in the adiabatic regime (Eagles, 1969; Emin and Holstein, 1976; Kalosakas et al. , 1998; Zheng and Avignon, 2003).

\section{E. All-coupling discrete polaron}

\section{Holstein model at any coupling}

During past twenty years significant efforts were directed towards the extension of the weak and strong-coupling perturbation lattice polaron theories to the intermediate region of the relevant parameters, $\lambda \sim 1$, and $\omega_{0} / t \sim 1$. It was argued (Alexandrov, 1992a; Firsov, 1975; Gogolin, 1982) that the expansion parameter is actually $1 / 2 z \lambda^{2}$, so the analytical strong-coupling expansion in powers of $1 / \lambda$ might have a wider region of applicability than one can expect using simple physical arguments (i.e. $\lambda>1$ ). However, it has not been clear how fast the expansion converges.

Kudinov and Firsov (1997) developed the analytical approach to the two-site Holstein model by the use of the expansion technique, which provides the electronic and vibronic terms as well as the wave functions and all correlation functions in any order of powers of $t$. They have found the exponential reduction factor in all orders of the $1 / \lambda$ perturbation expansion. On the other hand, the corrections to the atomic level were found as small as $1 / \lambda^{2}$ rather than exponential in agreement with the conventional second-order result, Eq.(175). Chatterjee and Das (2000) studied the same problem for any coupling within the perturbative expansion combined with MLF, Eq.(196), and MLF with a squeezing canonical 
transformation (Hang, 1988), $\exp (\tilde{S})$, where $\tilde{S}=\alpha\left(d_{i} d_{i}-d_{i}^{\dagger} d_{i}^{\dagger}\right)$. Using two variational parameters introduced by MLF and squeezing transformations allows for very good convergence of the $1 / \lambda$ perturbation series even in the near-adiabatic regime, $\omega_{0} / t \gtrsim 0.5$, where the conventional $1 / \lambda$ expansion shows poor convergence. These studies also showed that the region of the parameters of the Holstein model, where neither week nor strong-coupling perturbation analytical methods are applicable, is rather narrow. A semi-analytical approach to the solution of two coupled differential equations, Eq.(140]141), of the Holstein model in the whole parameter space has been proposed in (Han et al. , 2002) based on the coherentstate expansion of $u(x), v(x)$. These authors obtained the recursive relations among the expansion coefficients, allowing for highly accurate numerical solutions, which agree well with those by MLF method in the weak- and strong-coupling regimes. The deviation from the MLF solution in the intermediate-coupling regime implies that MLF misses some higher order correlation terms. A continued fraction analytical solution of the two-site Holstein model was derived by (Capone and Ciuchi, 2002) as for a related model in quantum optics (Swain, 1973). In practice it also requires some truncation of the infinite phonon Hilbert space. Finally all Green's functions for the two-site Holstein-Hubbard model (HHM) were derived in terms of continued fractions (Berciu, 2007).

Numerical results obtained by different methods actually show that the ground state energy (about $-E_{p}$ ) is not very sensitive to the parameters, while the effective mass and the bandwidth strongly depend on the polaron size, EPI range, and the adiabatic ratio, $\omega_{0} / t$. Several methods exist for numerical simulations of lattice polarons. They include exact diagonalisation (ED) (Alexandrov et al. , 1994a; Barisic, 2004; Capone et al. , 1997; Fehske et al. , 1995; Fehske and Trugman, 2007; Kongeter and Wagner, 1990; Marsiglio, 1993, 1995; Ranninger and Thibblin, 1992; Stephan, 1996; Wellein et al. , 1996), the global-local (GL) (Romero et al. , 1998) and other advanced variational methods ( $\mathrm{Ku}$ et al. , 2002; Trugman et al. , 2001), quantum Monte-Carlo (QMC) algorithms (Berger et al. , 1995; De Raedt and Lagendiik , 1982, 1983, 1984, 1985; Fradkin and Hirsch , 1983; Hirsch and Fradkin , 1982, 1983; Hohenadler et al. , 2004, 2005b; Hohenadler and von der Linden, 2007c), density matrix renormalization group (DMRG) (Jeckelmann and White, 1998; Jeckelmann et al., 1999; Zhang et al. , 1999), continuous-time QMC (Alexandrov and Kornilovitch, 1999; Hague et al. , 2006a; Kornilovitch , 1998, 1999; Spencer et al. , 2005), and diagrammatic QMC (Macridin et al. , 
2004; Mishchenko et al., 2000; Prokof'ev and Svistunov, 1998). The methods vary in accuracy and versatility, and, combined together, can provide all the polaron properties of interest in the entire space of model parameters. On the other hand, ED suffers from the necessary truncation of the phonon Hilbert space, especially at strong couplings and low phonon frequencies (even then, the total Hilbert space is huge, reducing the number of sites and leading to poor momentum resolution), DMRG cannot easily handle long-range interactions, diagrammatic QMC and ED are inconvenient in calculating the density of states, and path-integral CTQMC slows down at small frequencies (Fehske and Trugman , 2007; Hohenadler and von der Linden, 2007c; Kornilovitch , 2007; Mishchenko and Nagaosa, 2007). In numerical analysis of polaron models, a complex approach is needed where each method is employed to calculate what it does best.

Until recently most numerical studies were performed on the Holstein model (i.e with zero-range EPI). Reliable results for the intermediate region were obtained using ED of vibrating clusters (Alexandrov et al. , 1994a; Capone et al. , 1997; De Mello and Ranninger , 1997, 1998a; Fehske et al. , 1995; Fehske and Trugman,, 2007; Marsiglio, 1995; Ranninger and Thibblin, 1992; Stephan, 1996; Wellein et al. , 1996). Taking as a measure of the polaron kinetic energy the correlation function $t_{\text {eff }}=\left\langle-t\left(c_{1}^{\dagger} c_{2}+\right.\right.$ $\left.\left.c_{2}^{\dagger} c_{1}\right)\right\rangle$ (here $c_{1,2}$ are annihilation operators on the 'left' and 'right' sites of the Holstein model) one might doubt of the Lang-Firsov approach (De Mello and Ranninger,, 1997, 1998a), since this correlation function is much larger than the small polaron bandwidth. However, applying the $1 / \lambda$ expansion up to the second order in $t$ one obtains the numerical $t_{e f f}$ very close to the perturbation $t_{L F}$ in the strong-coupling regime, $\lambda>1$, (Alexandrov, 2000; Firsov et al. , 1999)

$$
t_{e f f} \simeq t_{L F} \equiv-t \exp \left(-\frac{\lambda t}{\omega}\right)-\frac{t}{\lambda}
$$

with $\lambda \equiv 2 E_{p} / t$. Here only the first exponential term describes the true coherent tunnelling, while the second term describes the correction to the middle of the polaron band owing to the virtual "back-forth" transitions to the neighbouring site, as discussed above (IV.B.1). The main contribution to $t_{\text {eff }}$ comes from the second-order term lowering the middle of the band (Alexandrov, 1992a, 2000; Gogolin , 1982; Kudinov and Firsov , 1997), rather than from the polaron-transport related first term (see also (De Mello and Ranninger, 1998b, 1999)). Comparing the analytical expression, Eq.(197) with the numerically calculated $t_{\text {eff }}$ one confirms that the Holstein-Lang-Firsov approach is asymptotically exact both in the 
non-adiabatic and adiabatic regimes (Alexandrov, 2000), if the second-order correction is taken into account.

The numerical diagonalisation of the two-site-one-electron Holstein model
(Alexandrov et al. , 1994a) shows that the first-order term of the $1 / \lambda$ perturbation theory describes well the polaron bandwidth in the non-adiabatic regime for all values of the coupling constant. There is no agreement in the adiabatic region, where the first order perturbation expression, Eq.(153), overestimates the polaron mass by a few orders of magnitude. A poor convergence of the perturbation expansion is due to appearance of the familiar double-well potential (Holstein , 1959b) in the adiabatic limit. The tunnelling probability is extremely sensitive to the shape of this potential. The splitting of levels for the two-site cluster is well described by the Holstein quasi-classical formula generalised for the intermediate coupling in Eq.(164). While the small Holstein polaron is only a few times heavier than the bare (unrenormalised) electron in a wide range of the coupling for a moderate adiabatic ratio $\omega_{0} / t \geq 1$, it becomes very heavy in the adiabatic regime and for the strong coupling (Alexandrov et al. , 1994a).

\section{Holstein polaron in infinite lattices}

A number of other independent numerical results proved that "by the use of the Holstein approximation and the canonical Lang-Firsov approach with appropriate corrections, one obtains an excellent estimate of the coherent bandwidth in both adiabatic and non-adiabatic (strong-coupling) regimes" (Fehske et al. ,1997). In particular the elaborate variational ED (VED) (Bonča et al. , 1999; Fehske and Trugman , 2007) provides an exact numerical solution of the Holstein crystal model, Eq.(195), in any dimension. In contrast to finite-lattice ED, it yields the ground-state energy which is a variational bound for the exact energy in the thermodynamic limit. Figure 11 shows the effective mass computed by VED (Bonča et al. 1999) in comparison with global-local (GL) and DMRG methods. $m^{*}$ is obtained from $m / m^{*}=(1 / 2 t) \partial^{2} E_{k} / \partial k^{2}, k \rightarrow 0$, where $m_{0}=1 /(2 t)$ is the rigid band mass (here the lattice constant is $a=1)$. In the intermediate coupling regime where VED gives an energy accuracy of 12 decimal places, one can calculate the effective mass accurately (6-8 decimal places) (Fehske and Trugman, 2007).

In Fig. 11 the parameters span different physical regimes including weak and strong 


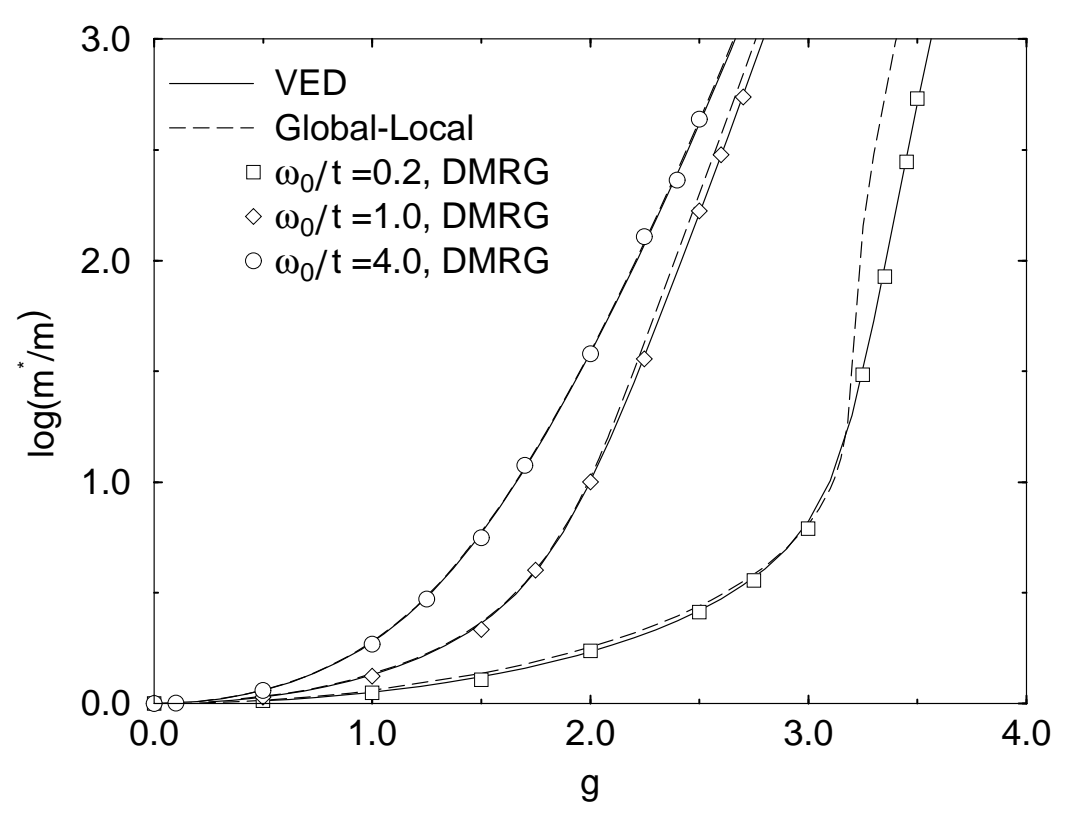

FIG. 11 Logarithm of the polaron effective mass in 1D Holstein model as a function of $g$ (after (Fehske and Trugman, 2007)). VED results (full lines) were obtained operating repeatedly $L=20$ times with the off-diagonal pieces of the Holstein Hamiltonian. For comparison GL (global-local) results (dashed lines) are included (Romero et al. , 1998). Open symbols, indicating the value of $\omega_{0} / t$, are DMRG results (Jeckelmann and White, 1998).

coupling, and low and high phonon frequency. There is good agreement between VED and GL away from strong coupling and excellent agreement in all regimes with DMRG results. DMRG calculations are not based on finite- $k$ calculations due to a lack of periodic boundary conditions, so they extrapolate the effective mass from the ground state data using chains of different sizes, which leads to larger error bars and demands more computational effort. There is no phase transition in the ground state of the model, but the polaron becomes extremely heavy in the strong coupling regime. The crossover to a regime of large polaron mass is more rapid in adiabatic regime, i.e. at small $\omega_{0} / t$.

Unifying systematization of the crossovers between the different polaron behaviors in one dimension was obtained in terms of quantum to classical, weak coupling to strong coupling, adiabatic to nonadiabatic, itinerant to self-trapped polarons and large to small polarons (O. Barisic and S. Barisic, 2008). It was argued that the relationship between various aspects of polaron states can be specified by five regimes: the weak-coupling regime, the regime of large adiabatic polarons, the regime of small adiabatic polarons, the regime of 
small nonadiabatic (Lang-Firsov) polarons, and the transitory regime of small polarons for which the adiabatic and nonadiabatic contributions are inextricably mixed in the polaron dispersion properties. Holstein polarons in three-dimensional anisotropic lattices have been recently studied using a variational ED technique, which provides highly accurate results for the polaron mass and polaron radius (Alvermann et al. , 2008). Varying the anisotropy (Alvermann et al. , 2008) demonstrated how a polaron evolves from a one-dimensional to a three-dimensional quasiparticle and showed that even the local Holstein interaction leads to an enhancement of anisotropy in charge-carrier motion.

For analyzing all-coupling polarons in more complex lattices the continuous-time pathintegral quantum Monte-Carlo algorithm (CTQMC) is ideally suited. The algorithm is formulated in real space (Kornilovitch , 1998, 2007) and based on the analytical integration over phonon degrees of freedom introduced by Feynman (1955) and on an earlier numerical implementation in discrete time by De Raedt and Lagendijk (1982,1983,1984,1985). CTQMC introduced two critical improvements. Firstly, formulation in continuous imaginary time eliminated errors caused by the Trotter slicing and made the method numerically exact for any strength of EPI. Secondly, introduction of twisted boundary conditions in imaginary time (Kornilovitch , 1998; Kornilovitch and Pike, 1997) enabled calculation of polaron effective masses, spectra and the densities of states (DOS) in any dimensions in infinite lattices.

The polaron action, obtained by analytical integration over phonon degrees of freedom, is a functional of the polaron path in imaginary time $\mathbf{r}(\tau)$. It is given by the following double integral

$$
\begin{gathered}
A[\mathbf{r}(\tau)]=\frac{z \lambda \bar{\omega}}{2 \Phi_{0}(0,0)} \int_{0}^{\bar{\beta}} \int_{0}^{\bar{\beta}} d \tau d \tau^{\prime-\bar{\omega} \bar{\beta} / 2}\left(e^{\bar{\omega}\left(\bar{\beta} / 2-\left|\tau-\tau^{\prime}\right|\right)}+e^{-\bar{\omega}\left(\bar{\beta} / 2-\left|\tau-\tau^{\prime}\right|\right)}\right) \Phi_{0}\left[\mathbf{r}(\tau), \mathbf{r}\left(\tau^{\prime}\right)\right] \\
+\frac{z \lambda \bar{\omega}}{\Phi_{0}(0,0)} \int_{0}^{\bar{\beta}} \int_{0}^{\bar{\beta}} d \tau d \tau^{\prime-\bar{\omega} \tau} e^{-\bar{\omega}\left(\bar{\beta}-\tau^{\prime}\right)}\left(\Phi_{\Delta \mathbf{r}}\left[\mathbf{r}(\tau), \mathbf{r}\left(\tau^{\prime}\right)\right]-\Phi_{0}\left[\mathbf{r}(\tau), \mathbf{r}\left(\tau^{\prime}\right)\right]\right): \\
\Phi_{\Delta \mathbf{r}}\left[\mathbf{r}(\tau), \mathbf{r}\left(\tau^{\prime}\right)\right]=\sum_{\mathbf{m}} \bar{f}_{\mathbf{m}}[\mathbf{r}(\tau)] \bar{f}_{\mathbf{m}+\Delta \mathbf{r}}\left[\mathbf{r}\left(\tau^{\prime}\right)\right]
\end{gathered}
$$

where the vector $\Delta \mathbf{r}=\mathbf{r}(\bar{\beta})-\mathbf{r}(0)$ is the difference between the end points of the polaron path, $\bar{\beta}=t / T, \bar{\omega}=\omega_{0} / t$, and $\bar{f}_{\mathbf{m}}(\mathbf{n})=f_{\mathbf{m}}(\mathbf{n}) / \kappa$ (see also Eq.(194) $)$. From this starting point, the polaron is simulated using the Metropolis Monte-Carlo method. The electron path is continuous in time with hopping events (or kinks) introduced or removed from the 
path with each Monte-Carlo step. From this ensemble, various physical properties may be computed, in particular, the ground state energy, the number of phonons in the polaron "cloud" and the polaron band energy spectrum,

$$
\epsilon_{\mathbf{k}}-\epsilon_{0}=-\lim _{\bar{\beta} \rightarrow \infty} \frac{1}{\bar{\beta}} \ln \langle\cos (\mathbf{k} \cdot \Delta \mathbf{r})\rangle:
$$

where $\mathbf{k}$ is the quasi momentum. By expanding this expression in small $\mathbf{k}$, the $i$-th component of the inverse effective mass is obtained as

$$
\frac{1}{m_{i}^{*}}=\lim _{\bar{\beta} \rightarrow \infty} \frac{1}{\bar{\beta}}\left\langle\left(\Delta \mathbf{r}_{i}\right)^{2}\right\rangle: .
$$

Thus the inverse effective mass is the diffusion coefficient of the polaron path in the limit of the infinitely long "diffusion time" $\bar{\beta}$. CTQMC has enabled accurate analysis of models with long-range electron-phonon interactions (Alexandrov and Kornilovitch, 1999; Hague et al. 2006a; Spencer et al. , 2005) and a model with anisotropic electron hopping (Kornilovitch, 1999).

All numerical results confirm gross polaronic features well understood analytically by (Holstein , 1959b) and others both in the non-adiabatic and adiabatic regimes. A great power of numerical methods is the ability to calculate an entire polaron spectrum, and the polaron DOS $\rho(E)=N^{-1} \sum_{\mathbf{k}} \delta\left(E-E_{\mathbf{k}}+E_{0}\right)$, in the whole parameter space. The coherent part of the spectrum, $\epsilon_{\mathbf{k}}$, possesses an interesting property of flattening at large lattice momenta in the adiabatic limit, $t \gg \omega_{0}$ (Fehske and Trugman ), 2007; Romero et al. , 1998, 1999; Stephan, 1996; Wellein et al. , 1996; Wellein and Fehske, 1997). In the weakcoupling limit, the flattening can be readily understood as hybridization between the bare electron spectrum and a phonon mode (Levinson and Rashba, 1973). The resulting polaron dispersion is cosine-like at small $\mathbf{k}$ and flat at large $\mathbf{k}$. As a result the polaron DOS should be peaked close to the top of the polaron band. Exact VED (Fehske and Trugman, 2007) and CTQMC (Kornilovitch , 2007) calculations have confirmed that this is indeed the case for the short-range EPI. At small $\omega_{0}$, DOS develops a massive peak at the top of the band. With increasing $\omega_{0}$, the polaron spectrum approaches the cosine-like shape in full accordance with the Lang-Firsov non-adiabatic formula. The respective DOS gradually assumes the familiar shape of the tight-binding band with renormalised hopping integrals. However, at small-tomoderate $\omega_{0} / t$ in two and three dimensions, the bottom half of the polaron band contains a tiny minority of the total number of states, so that the system's responses will be dominated by the states in the peak. 
In the long-range model (194) DOS is much closer to the tight-binding shape than the Holstein DOS at the same parameters (Hague et al. , 2006a). The polaron spectrum and DOS is another manifestation of the extremity of the Holstein model. Long-range EPI removes those peculiarities and makes the shape of polaron bands closer to the $1 / \lambda$ expansion results.

\section{Discrete Fröhlich polaron at any coupling}

As discussed above (IV.C) the lattice polaron mass strongly depends on the radius of EPI. So does the range of the applicability of the analytical $1 / \lambda$ expansion theory. The theory appears almost exact in a wide region of the Fröhlich EPI, Eq.(194), for which the exact polaron mass was calculated with CTQMC algorithm in (Alexandrov and Kornilovitch, 1999).

At large $\lambda(>1.5)$ SFP was found to be much lighter than SHP in agreement with the analytical results (IV.C), while the large Fröhlich polaron (i.e. at $\lambda<1$ ) was heavier than the large Holstein polaron with the same binding energy (Alexandrov and Kornilovitch, 1999). The mass ratio $m_{F P}^{*} / m_{H P}^{*}$ is a non-monotonic function of $\lambda$. The effective mass of the small Fröhlich polaron, $m_{F P}^{*}(\lambda)$ is well fitted by a single exponent, which is $e^{0.73 \lambda}$ for $\omega_{0}=t$ and $e^{1.4 \lambda}$ for $\omega_{0}=0.5 t$. The exponents are remarkably close to those obtained with the Lang-Firsov transformation, $e^{0.78 \lambda}$ and $e^{1.56 \lambda}$, respectively. Hence, in the case of the Fröhlich interaction the transformation is perfectly accurate even in the moderate adiabatic regime, $\omega_{0} / t \leq 1$ for any coupling strength. It is not the case for the Holstein polaron. If the interaction is short-ranged, the same analytical technique is applied only in the non-adiabatic regime $\omega_{0} / t>1$.

An important question about polaron properties also involves the effects of screening on the electron-phonon interaction. Unscreened EPI makes polarons very mobile (Alexandrov, 1996), which leads to strong effects even on the qualitative physical properties of the polaron gas. Modeling the screening effects, the interaction force between electrons and phonons was

introduced in (Spencer et al. , 2005) in the form of a screened discrete Fröhlich interaction,

$$
f_{\mathbf{m}}(\mathbf{n})=\frac{\kappa}{\left[(\mathbf{m}-\mathbf{n})^{2}+1\right]^{3 / 2}} \exp \left(-\frac{|\mathbf{m}-\mathbf{n}|}{R_{\mathrm{sc}}}\right) .
$$

It describes EPI of holes with c-axis polarised lattice distortions, which has been suggested 
as the relevant electron-phonon interaction in the cuprates (Alexandrov, 1996).

The CTQMC polaron mass for the one-dimensional lattice with the screened Fröhlich EPI, Eq.(202) is shown in Fig,12 at four different values of the screening length, $R_{\mathrm{sc}} \rightarrow 0$ (the short-range Holstein interaction), $R_{\mathrm{sc}}=1, R_{\mathrm{sc}}=3$, and $R_{\mathrm{sc}} \rightarrow \infty$ (the non-screened Fröhlich interaction).

An important observation is evident from the plot of $\ln \left(m^{*} / m_{0}\right)$ against $\lambda$ shown in figure (12). At intermediate and large couplings (that is, in the transition and small polaron regions), altering the value of $R_{\mathrm{sc}}$ has a dramatic effect on the effective mass. For example, the non-screened Fröhlich polaron is over $10^{3}$ times "lighter" than the Holstein polaron at $\lambda=4$, and over $10^{4}$ times lighter at $\lambda=5$. It is apparent from the above results for the screened Fröhlich model that as $R_{\mathrm{sc}}$ increases (from Holstein to Fröhlich), the QMC results move, in general, closer to the " $1 / \lambda$ " expansion over the entire range of $\lambda$. That is, the " $1 / \lambda "$ expansion becomes generally more applicable as the range of EPI increases (as well as with increasing $\bar{\omega})$.

The polaron features for the long-range EPI were also investigated by extending a variational approach previously proposed for the study of systems with local (Holstein) coupling (Perroni et al., 2004). The ground state spectral weight, the average kinetic energy, the mean number of phonons, and the electron-lattice correlation function were calculated for a wide range of model parameters focusing on the adiabatic regime. A strong mixing of electronic and phononic degrees of freedom even for small values of EPI was found in the adiabatic case due to the long-range interaction.

\section{F. Isotope effect on the polaron mass and the polaron band-structure}

There is a qualitative difference between ordinary metals and polaronic (semi)conductors. The renormalised effective mass of electrons is independent of the ion mass $M$ in ordinary metals (where the Migdal adiabatic approximation is believed to be valid, see below), because $\lambda$ does not depend on the isotope mass. However, when electrons form polarons dressed by lattice distortions, their effective mass $m^{*}$ depends on $M$ through $m^{*}=m \exp \left(\gamma E_{p} / \omega_{0}\right)$, in the strong-coupling limit. Here the phonon frequency depends on the ion mass, so that there is a large polaronic isotope effect (PIE) on the carrier mass with the carrier mass isotope exponent $\alpha_{m^{*}}=(1 / 2) \ln \left(m^{*} / m\right)$ as predicted in (Alexandrov, 1992b), in contrast 


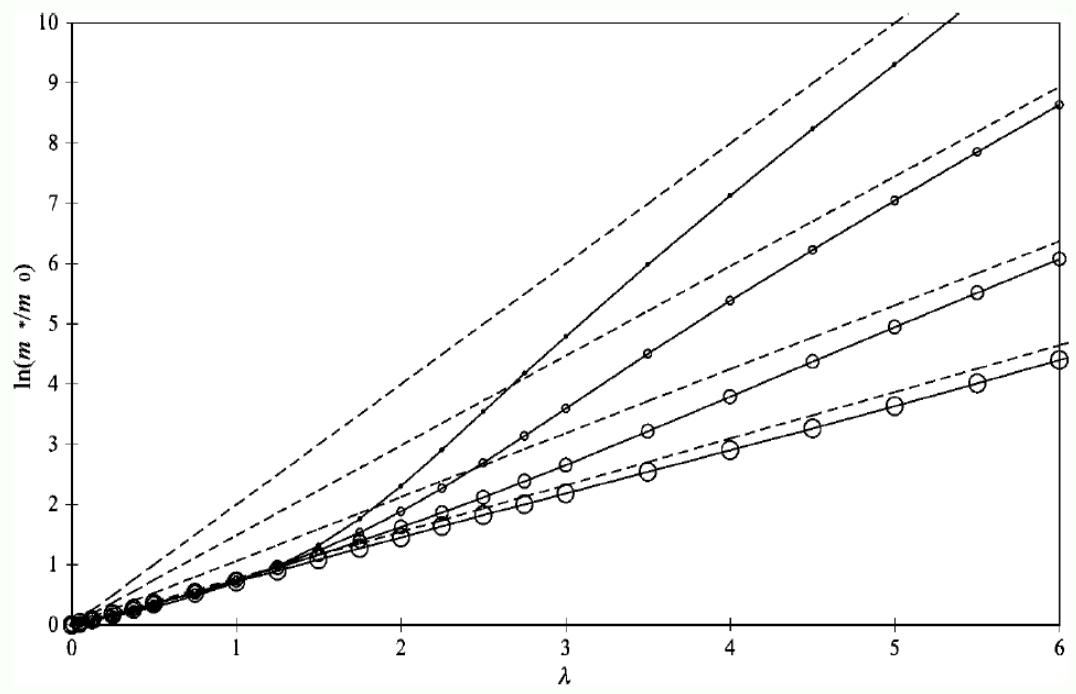

FIG. 12 The logarithm of the effective mass for screening lengths $R_{\mathrm{sc}}=0,1,3, \infty$ (increasing size of circles) versus $\lambda$ at $\bar{\omega}=1$ (Spencer et al. , 2005). At intermediate and strong coupling, decreasing the value of $R_{\mathrm{sc}}$ dramatically increases the effective mass. The curves tend to the strong-coupling analytical result (dashed lines).

to the zero isotope effect in ordinary metals (see (Alexandrov, 2003) for more details). The effect was found experimentally in cuprates (Khasanov et al. , 2004; Zhao and Morris , 1995; Zhao et al. , 1997) and manganites (Alexandrov et al. , 2001). More recent high resolution angle resolved photoemission spectroscopy (ARPES) also revealed a complicated isotope effect on the whole band-structure in cuprate superconductors depending on the electron energy and momentum (Gweon et al. , 2004).

PIE in the intermediate region of parameters was calculated using the dynamic meanfield approximation (DMFT) (Deppeler and Millis , 2002; Paci et al. , 2005), and CTQMC algorithm (Hague et al. ., 2006a; Kornilovitch and Alexandrov, 2004; Spencer et al. , 2005). Importantly $\alpha_{m^{*}}$ and the effective mass averaged over dimensions are related to the critical temperature isotope exponent, $\alpha=-d \ln T_{c} / d \ln M$, of a (bi)polaronic superconductor as

$$
\alpha=\alpha_{m^{*}}\left(1-\frac{m / m^{*}}{\lambda-\mu_{c}}\right),
$$

where $\mu_{c}$ is the Coulomb pseudo-potential (Alexandrov, 1992b, 2003). 

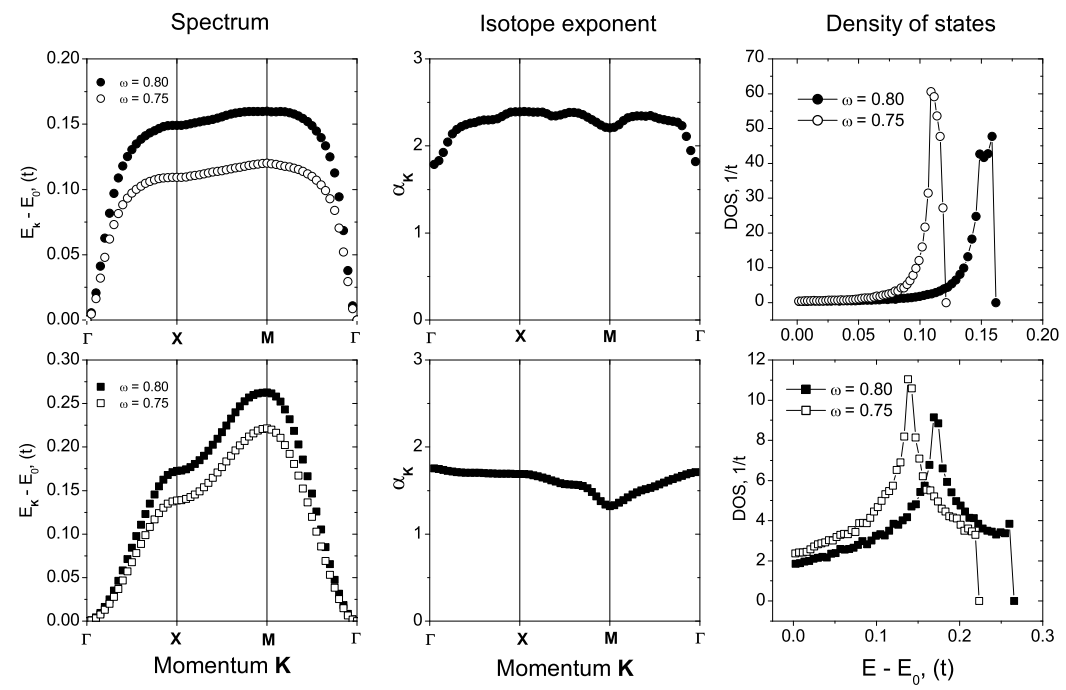

FIG. 13 Isotope effect on polaron spectrum and density of states. Top row: the $2 d$ Holstein polaron at $\lambda=1.2$. Left: polaron spectrum at $\omega=0.80$ and 0.75 (in units of $t$ ). Middle: the isotope exponent for each k-point. Right: the density of states for the two frequencies. Bottom row: the same for the discreate $2 d$ Fröhlich polaron at $\lambda=2.4$ (after (Kornilovitch and Alexandrov), 2004)).

In the adiabatic regime the isotope effect on the polaron mass does not fully represent the isotope effect on the vast majority of polaron states, in particular for the short-range EPI, so that additional insight can be gained from the isotope effect on the entire polaron spectrum (Kornilovitch and Alexandrov, 2004). The isotope effect on polaron spectrum and DOS in $d=2$ is illustrated in Fig 13. The ratio of the two phonon frequencies, $\omega=0.80 t$ and $0.75 t$, has been chosen to roughly correspond to the substitution of ${ }^{16} \mathrm{O}$ for ${ }^{18} \mathrm{O}$ in complex oxides. One can see that the polaron band shrinks significantly, by 20-30\%, for both polaron types. The middle panels show the isotope exponents on spectrum points calculated as

$$
\alpha_{\mathbf{k}}=\frac{1}{2} \frac{\langle\omega\rangle}{\left\langle\epsilon_{\mathbf{k}}\right\rangle} \frac{\Delta \epsilon_{\mathbf{k}}}{\Delta \omega}
$$

where the angular brackets denote the mean value of either the two frequencies or of the two energy values. An interesting observation is that $\alpha_{\mathbf{k}}$ of the Fröhlich polaron is roughly independent of $\mathbf{k}( \pm 10 \%)$. In the Holstein case $\alpha_{\mathbf{k}}$ dips in the vicinity of the $\Gamma$ point.

Unconventional isotope effects as observed in high-temperature superconducting cuprates (Khasanov et al. , 2004), were also explained by polaron formation stemming from the 
coupling to the particular quadrupolar Q(2)-type phonon mode (Bussmann-Holder et al. 2005). Polaronic isotope effects in the spectral function of strongly correlated systems were numerically studied by (Fratini and Ciuchi ,2005) in the framework of the Holstein-Hubbard model and by (Mishchenko and Nagaosa, 2006) using the extended $t-J$ model including EPI.

\section{G. Jahn-Teller polaron}

The density-displacement EPIs discussed above are not only possible types of the electronlattice coupling. Examples of other types are the Su-Schrieffer-Heeger (SSH) EPI (Su et al. , 1979) ( for recent path-integral results for SSH polarons see (Zoli , 2007)), and the JahnTeller (JT) EPI (Jahn and Teller, 1937). In the former, the lattice deformation is coupled to electron kinetic energy, while JT involves a multidimensional electron basis and a multidimensional representation of the deformation group. The JT interaction is active in some molecules and crystals of high point symmetry, and it has served as a guide in the search for high-temperature superconductivity (Bednorz and Müller, 1988). Later on JT-type EPIs were widely discussed in connection with cuprate and other high- $\mathrm{T}_{c}$ superconductors (see, for example, (Alexandrov et al. , 1996; Gunnarsson, 1997; Mertelj et al. , 2005; Mihailović and Kabanov,,2001; Müller , 2000)) and colossal-magnetoresistance manganites (see, for example, Refs. (Bussmann-Holder and Bishop, 1999; Edwards, 2002; Millis et al. , 1995; [Röder et al. , 1996; Yunoki et al. , 1998b)).

The simplest model of the JT interaction is the $E \otimes e$ interaction (Kanamori, 1960) that describes a short-range coupling between twice-degenerate $e_{g}$ electronic levels $\left(c_{1}, c_{2}\right)$ and a local double-degenerate vibron mode $(\zeta, \eta)$. The Hamiltonian reads ( here we follow (Kornilovitch , 2007))

$$
\begin{aligned}
H_{\mathrm{JT}}= & -t \sum_{\left\langle\mathbf{n n}^{\prime}\right\rangle}\left(c_{\mathbf{n}^{\prime} 1}^{\dagger} c_{\mathbf{n} 1}+c_{\mathbf{n}^{\prime} 2}^{\dagger} c_{\mathbf{n} 2}\right) \\
& -\kappa \sum_{\mathbf{n}}\left[\left(c_{\mathbf{n} 1}^{\dagger} c_{\mathbf{n} 2}+c_{\mathbf{n} 2}^{\dagger} c_{\mathbf{n} 1}\right) \eta_{\mathbf{n}}+\left(c_{\mathbf{n} 1}^{\dagger} c_{\mathbf{n} 1}-c_{\mathbf{n} 2}^{\dagger} c_{\mathbf{n} 2}\right) \zeta_{\mathbf{n}}\right] \\
& +\sum_{\mathbf{n}}\left[-\frac{1}{2 M}\left(\frac{\partial^{2}}{\partial \zeta_{\mathbf{n}}^{2}}+\frac{\partial^{2}}{\partial \eta_{\mathbf{n}}^{2}}\right)+\frac{M \omega^{2}}{2}\left(\zeta_{\mathbf{n}}^{2}+\eta_{\mathbf{n}}^{2}\right)\right]
\end{aligned}
$$

The symmetry of the interaction ensures the same coupling parameter $\kappa$ for the two phonons. Because the ionic coordinates of different cells are not coupled, the model describes a collec- 
tion of separate clusters that are linked only by electron hopping. To relate the Hamiltonian to more realistic situations, phonon dispersion should be added (Allen and Perebeinos, 1999; Mihailović and Kabanov, 2001).

An important property of the $E \otimes e$ interaction is the absence of an exact analytical solution in the atomic limit $t=0$. Here, in contrast with the Holstein and Fröhlich EPI, the atomic limit is described by two coupled partial differential equations for the electron doublet $\psi_{1,2}(\zeta, \eta)$. At large couplings, however, the elastic energy assumes the Mexican hat shape and the phonon dynamics separates into radial oscillatory motion and azimuthal rotary motion. This results in an additional pre-exponential factor $\propto \kappa$ in the ion overlap integral, leading to the effective mass $m_{\mathrm{JT}}^{*} / m=(2 / \pi g)^{1 / 2} \exp g^{2}$, where $g^{2}=\kappa^{2} / 2 M \omega^{3}$ (Takada), 2000).

A path integral approach to Hamiltonian (205) was developed by Kornilovitch (2000). Its details are found in (Kornilovitch , 2007). Because there are two electron orbitals, the electron path must be assigned an additional orbital index (or colour) $a=1,2$. Colour 1 (or 2) of a given path segment means that it resides in the first (second) atomic orbital of the electron doublet. There is a difference between the two phonons. Phonon $\zeta$ is coupled to electron density, like in the Holstein case. The difference from the Holstein is that the direction of the force changes to the opposite when the electron changes orbitals. In contrast, phonon $\eta$ is coupled to orbital changes themselves: the more often the electron changes orbitals, the more "active" is $\eta$. Discrete orbital changes are analogous to electron hops between discrete lattice sites, and as such are associated with "kinetic orbital energy". Phonon coupling to orbital changes is analogous to phonon coupling to electron hopping in the SSH model (Su et al. , 1979).

The JT polaron properties can be calculated with no approximations using QMC algorithm. The effective mass, spectrum, and density of states are obtained as for the conventional lattice polarons. Most properties behave similarly to those of the $d=3$ Holstein polaron at the same phonon frequency (Kornilovitch, 2000). For example, the kinetic energy sharply decreases by absolute value between $\lambda=1.2$ and 1.4. The JT polaron mass is slightly larger at the small to intermediate coupling, but several times smaller at the strong coupling. This non-monotonic behaviour of the ratio of the JT and Holstein masses was later confirmed by accurate variational calculations (El Shawish et al. , 2003), although in that work the JT polaron (and bipolaron) was investigated in one spatial dimension. The relative 
lightness of the JT polaron is consistent with Takada's result mentioned above (Takada), 2000). Finally, the density of JT polaron states features the same peak at the top of the band, caused by the spectrum flattening at large polaron momenta, see Fig. (13).

In summary, the local character of the JT interaction and the independence of vibrating clusters result in the same extremity of polaron properties as in the $3 d$ Holstein model. One could expect that either a long-range JT interaction or phonon dispersion will soften the sharp polaron features and make JT polarons more mobile (Kornilovitch, 2007).

\section{RESPONSE OF DISCRETE POLARONS}

\section{A. Hopping mobility}

Studies of lattice polarons allowed for a theoretical understanding of low mobility problem (Ioffe, 1957) of many "poor" conductors, where an estimate of the mean-free path yields values much lower than the lattice constant. Transport properties of lattice polarons depend strongly on temperature. For $T$ lower than the characteristic phonon energy polaron kinetics is the Boltzmann kinetics of heavy particles tunnelling in the narrow band (Holstein , 1959b). However at higher temperatures the polaron band collapses (IV.B.2), and the transport is diffusive via thermally activated jumps of polarons from site to site (Appel), 1968; Firsov 1975; Holstein, 1959b; Klinger, 1979; Lang and Firsov, 1963, 1968; Mott and Davis, 1982; Yamashita and Kurosawa, 1958).

The $1 / \lambda$ perturbation expansion is particularly instrumental in calculating hopping mobilities and optical absorption coefficients. Applying the canonical transformation (IV.B) and singling out the diagonal coherent tunnelling in the polaron band one can write the Hamiltonian as

$$
\tilde{H}=H_{p}+H_{p h}+H_{i n t}
$$

where $H_{p}=\sum_{\mathbf{k}} \xi_{\mathbf{k}} c_{\mathbf{k}}^{\dagger} c_{\mathbf{k}}$ is the "free" polaron contribution (here we drop the spin), $H_{p h}=$ $\sum_{\mathbf{q}} \omega_{\mathbf{q}}\left(d_{\mathbf{q}}^{\dagger} d_{\mathbf{q}}+1 / 2\right)$ is the phonon part, and $\xi_{\mathbf{k}}=Z^{\prime} E_{\mathbf{k}}-\mu$ is the renormalised (polaron) band dispersion. The chemical potential $\mu$ includes the polaron level shift $-E_{p}$, and it could also include all higher orders in $1 / \lambda$ corrections to the polaron spectrum, which are independent 
of $\mathbf{k}$, Eq.(175). The band-narrowing factor $Z^{\prime}$ is defined as

$$
Z^{\prime}=\frac{\sum_{\mathbf{m}} t(\mathbf{m}) e^{-g^{2}(\mathbf{m})} \exp (-i \mathbf{k} \cdot \mathbf{m})}{\sum_{\mathbf{m}} t(\mathbf{m}) \exp (-i \mathbf{k} \cdot \mathbf{m})}
$$

which is $\left.Z^{\prime}=\exp \left(-\gamma E_{p} / \omega\right)\right)$ where $\gamma \leq 1$ depends on the range of EPI and phonon frequency dispersions (IV.Cl). The interaction term $H_{\text {int }}$ comprises the polaron-polaron interaction, Eq.(171), and the residual polaron-phonon interaction

$$
H_{p-p h} \equiv \sum_{i \neq, j}\left[\hat{\sigma}_{i j}-\left\langle\hat{\sigma}_{i j}\right\rangle\right] c_{i}^{\dagger} c_{j},
$$

where $\left\langle\hat{\sigma}_{i j}\right\rangle$ means averaging with respect to the bare phonon distribution. In the framework of the single-polaron problem one neglects the polaron-polaron interaction and treats $H_{p-p h}$ as a perturbation.

The motion of the small polaron at high temperatures is a random walk consisting of steps from site to site (Holstein, 1959b). Holstein calculated the probability $W$ for the hop of a small polaron to a neighbouring site. He suggested that the random walk was a Markovian process. For such processes the diffusion coefficient is given by $D \simeq a^{2} W$, where $W$ is the hopping probability. The only term in the polaronic Hamiltonian, which changes the phonon occupation numbers, is the polaron-phonon interaction $H_{p-p h}$. The nearest neighbour hopping probability in the second order is

$$
W=2 \pi\left\langle\sum_{j}\left|\left\langle j\left|H_{p-p h}\right| i\right\rangle\right|^{2} \delta\left(\sum_{\mathbf{q}} \omega_{\mathbf{q}}\left(n_{\mathbf{q}}^{j}-n_{\mathbf{q}}^{i}\right)\right)\right\rangle
$$

where $|i\rangle$ and $|j\rangle$ are the eigenstates of $H_{0}$ corresponding to the polaron on site $i$ with $n_{\mathbf{q}}^{i}$ phonons in each phonon mode and the polaron on the neighbouring site $j$ with $n_{\mathbf{q}}^{j}$ phonons, respectively. Replacing the $\delta$ function in Eq.(209) by the integral yields

$$
W=t^{2} e^{-2 g^{2}} \int_{-\infty}^{\infty} d \tau\left[\exp \left(\frac{1}{N} \sum_{\mathbf{q}}|\gamma(\mathbf{q})|^{2}[1-\cos (\mathbf{q} \cdot \mathbf{a})] \frac{\cos \left(\omega_{\mathbf{q}} \tau\right)}{\sinh \frac{\omega_{\mathbf{q}}}{2 T}}\right)-1\right] e^{-i 0^{+}|\tau|} .
$$

The integration over $\tau$ is performed using a saddle-point approximation by allowing a finite phonon frequency dispersion $\delta \omega \ll \omega_{\mathbf{q}}$. Expanding $\cos \left(\omega_{\mathbf{q}} \tau\right)$ in powers of $\tau$, and using the Einstein relation $\mu=e D / T$ one obtains the hopping mobility,

$$
\mu_{h}=e a^{2} \frac{\pi^{1 / 2} t^{2}}{2 T\left(E_{a} T\right)^{1 / 2}} e^{-E_{a} / T} .
$$


where

$$
E_{a}=\frac{T}{N} \sum_{\mathbf{q}}|\gamma(\mathbf{q})|^{2}[1-\cos (\mathbf{q} \cdot \mathbf{a})] \tanh \left(\omega_{\mathbf{q}} / 4 T\right)
$$

is the activation energy, which is half of the polaron level shift $E_{p}$ for the Holstein EPI. This expression is applicable at $T \gg \omega_{0} / 2$ and $t^{2} / \omega_{0}\left(E_{a} T\right) \ll 1$. Importantly, increasing the EPI range diminishes the value of $E_{a}$ further. The hopping mobility $\mu_{h} \equiv \sigma_{h} /$ ne $\sim \exp \left(-E_{a} / T\right)$ can be below $e a^{2} \simeq 1 \mathrm{~cm}^{2} / V s$, which is the lowest limit for the Boltzmann theory to be applied. Within the Boltzmann theory such a low mobility corresponds to the mean free path $l<a$, which is not a reasonable result.

Holstein suggested that at low temperatures $T<\omega_{0} / 2 \ln \gamma_{0}$, there is the ordinary Boltzmann transport in momentum space, but in the narrow polaron band. According to (Holstein , 1959b), the transition from the band regime to the hopping regime occurs when the uncertainty in the polaron energy becomes comparable to the width of the polaron band. If phonons dominate in the scattering, the polaron mobility, $\mu=\mu_{t}+\mu_{h}$ decreases when the temperature increases from zero to some $T_{\min }$, where it is at minimum, because its Boltzmann part, $\mu_{t}$, falls down due to an increasing number of phonons, while the hopping part, $\mu_{h}$, remains small. However $\mu$ increases above $T_{\min }$, due to the thermal activated hopping. There is a wide temperature range around $T_{\min }$ where the thermal activated hopping still makes a small contribution to the conductivity, but the uncertainty in the polaron band is already significant (Firsov, 1975, 2007). The polaron transport theory requires a special diagrammatic technique (Firsov, 1975, 2007; Lang and Firsov, 1963, 1968) and a conditional-probability function description (Kudinov and Firsov, 1965, 1966) in this region.

The transverse conductivity $\sigma_{x y}$ and the Hall coefficient $R_{H}=\sigma_{x y} / H \sigma_{x x}^{2}$ of lattice polarons can be calculated with the Peierls substitution (Peierls , 1933),

$$
t(\mathbf{m}-\mathbf{n}) \rightarrow t(\mathbf{m}-\mathbf{n}) e^{-i e \mathbf{A}(\mathbf{m}) \cdot(\mathbf{m}-\mathbf{n})}
$$

which is a fair approximation if the magnetic field, B, is weak compared with the atomic field $e B a^{2}<<1$. Here $\mathbf{A}(\mathbf{r})$ is the vector potential, which can be also time dependent. Within the Boltzmann theory the sign of the Hall coefficient $R_{H} \simeq \pm 1 /$ en depends on the type of carriers (holes or electrons) and the Hall mobility $\mu_{H} \equiv R_{H} \sigma_{x x}$ is the same as the drift mobility $\mu_{t}$ up to a numerical factor of the order of 1 . The calculations of the hopping Hall current similar to those of the hopping conductivity (Böttger et al. , 2007; Bryksin and Firsov, 1970; Emin, 
1971; Friedman and Holstein , 1963) show that the Hall mobility depends on the symmetry of the crystal lattice and has nothing in common with the hopping mobility, neither with respect to the temperature dependence and even nor with respect to the sign. In particular for hexagonal lattices three-site hops yield (Friedman and Holstein, 1963)

$$
\mu_{H}=e a^{2} \frac{\pi^{1 / 2} t}{\left(12 E_{a} T\right)^{1 / 2}} e^{-E_{a} / 3 T}
$$

with the same sign for electrons and holes. The activation energy of the Hall mobility is three times less than that of the hopping mobility. In cubic crystals, the hopping Hall effect is governed by four-site hops. The four-site calculations in (Bryksin and Firsov, 1970; Emin, 1971) gave the Hall mobility with the "normal" sign depending on the type of carriers.

The Hall conductivity and resistivity of strongly localized electrons at low temperatures and small magnetic fields strongly depend on frequency, the size of a sample (Entin-Wohlman et al. , 1995), and on a magnetic order like, for example in ferromagnetic (Ga,Mn)As (Burkov and Balents, 2003). In the presence of the spin-orbit interaction each hopping path acquires a spin-dependent phase factor of the same form as that in a perpendicular (to the 2D system) magnetic field, which leads to spin accumulation and spin-Hall effects (Böttger et al. ., 2007; Entin-Wohlman et al. , 2005).

\section{B. Optical conductivity}

One of the fingerprints of lattice polarons is the frequency $(\omega)$ and temperature dependence of their mid-infrared (MIR) conductivity $\sigma(\omega)$. In the low frequency and low temperature region, where the tunneling band transport operates (Holstein, 1959a,, b), the conductivity acquires the conventional Drude form,

$$
\sigma(\omega)=\frac{n e \mu_{t}}{1+(\omega \tau)^{2}}
$$

where the transport relaxation time $\tau$ may be frequency dependent because of the narrow band. For high (mid-infrared) frequencies, well above the polaron bandwidth $(\omega \gg w)$ but below the interband gap, the Drude law is not applied. In this frequency region one can apply the generalised Einstein relation $\sigma(\omega)=e D(\omega) / \omega$, where $D(\omega)=a^{2} W(\omega)$, and $W(\omega)$ is the hopping probability of the absorption of the energy quantum $\omega$ (Holstein, 1959b). The number of nearest neighbour transitions per second with the absorption of a photon of 
the energy $\omega$ is given by the Fermi-Dirac golden rule,

$$
W^{-}=2 \pi\left\langle\sum_{j}\left|\left\langle i\left|H_{p-p h}\right| j\right\rangle\right|^{2} \delta\left(\sum_{\mathbf{q}} \omega_{\mathbf{q}}\left(n_{\mathbf{q}}^{j}-n_{\mathbf{q}}^{i}\right)+\omega\right)\right\rangle,
$$

and with the emission,

$$
W^{+}=2 \pi\left\langle\sum_{j}\left|\left\langle i\left|H_{p-p h}\right| j\right\rangle\right|^{2} \delta\left(\sum_{\mathbf{q}} \omega_{\mathbf{q}}\left(n_{\mathbf{q}}^{j}-n_{\mathbf{q}}^{i}\right)-\omega\right)\right\rangle .
$$

As a result one obtains

$$
\begin{aligned}
W(\omega) & =W^{-}-W^{+}=2 t^{2} e^{-2 g^{2}} \sinh (\omega / 2 T) \\
& \times \int_{-\infty}^{\infty} d \tau e^{-i \omega \tau}\left[\exp \left(\frac{1}{N} \sum_{\mathbf{q}}|\gamma(\mathbf{q})|^{2}[1-\cos (\mathbf{q} \cdot \mathbf{a})] \frac{\cos (\omega \tau)}{\sinh \omega_{\mathbf{q}} / 2 T}\right)-1\right] .
\end{aligned}
$$

As in the case of the hopping mobility discussed above the integral over $\tau$ is calculated using the saddle-point approximation (Böttger and Bryksin, 1985),

$$
\sigma(\omega)=\sigma_{h} \frac{T \sinh (\omega / 2 T)}{\omega} e^{\omega^{2} / 4 \delta}
$$

where

$$
\delta=\frac{1}{2 N} \sum_{\mathbf{q}}|\gamma(\mathbf{q})|^{2}[1-\cos (\mathbf{q} \cdot \mathbf{a})] \frac{\omega_{\mathbf{q}}^{2}}{\sinh \left(\omega_{\mathbf{q}} / 2 T\right)} .
$$

For high temperatures, $T>>\omega_{0} / 2$, this expression simplifies as

$$
\sigma(\omega)=n e^{2} a^{2} \frac{\pi^{1 / 2} t^{2}\left[1-e^{-\omega / T}\right]}{2 \omega\left(E_{a} T\right)^{1 / 2}} \exp \left[-\frac{\left(\omega-4 E_{a}\right)^{2}}{16 E_{a} T}\right] .
$$

Here $\sigma_{h}=n e \mu_{h}$ and $n$ is the atomic density of polarons. The frequency dependence of the MIR conductivity has a form of an asymmetric Gaussian peak centered at $\omega=4 E_{a}$ with the half-width $4 \sqrt{E_{a} T}$ (Eagles , 1963; Klinger, 1963; Reik, 1963). According to the Franck-Condon principle, the position of the ions is not changed during an optical transition. Therefore the frequency dependence of the MIR conductivity can be understood in terms of transitions between the adiabatic levels of the two-site Holstein model (IV.A.2). The polaron, say, in the left potential well absorbs a photon through the vertical transition to the right well, where the deformation is lacking, without any change in the molecular configuration. The photon energy required to excite the polaron from the bottom of the well is $\omega \simeq 2 E_{p}$, which corresponds to the maximum of $\sigma(\omega)$ in Eq.(221). The main contribution to the absorption comes from the states near the bottom with the energy of the order of 
$T$. The corresponding photon energies are found in the interval $2 E_{p} \pm \sqrt{8 E_{p} T}$ in agreement with Eq.(221). For low temperatures $T<\omega_{0} / 2$ the half-width of the MIR maximum is about $\sim \sqrt{E_{p} \omega_{0}}$ rather than $\sim \sqrt{E_{p} T}$ (Emin, 1993). The optical absorption of small polarons is distinguished from that of large polarons (III.F) by the shape and the temperature dependence. Their comparison (Calvani, 2001; Emin , 1973, 1993) shows a more asymmetric and less temperature dependent mid-infrared (MIR) absorption of large polarons compared with that of small polarons. The high-frequency behavior of the optical absorption of small polarons is described by a Gaussian decay (Reik , 1972), Eq.(221), while for large polarons it is much slower power law $\omega^{-5 / 2}$ (Devreese, 1972).

Many materials are characterised by intermediate values of EPI, $\lambda \sim 1$, which requires an extension of the theory of optical absorption to the crossover region from continuous to lattice polarons. The intermediate coupling and frequency regime has been inaccessible for an analytical or semi-analytical analysis, with an exception of infinite spatial dimensions, where DMFT yields reliable results (Benedetti and Zeyher, 1998; Ciuchi et al. , 1997; Fratini and Ciuchi, 2003; Sumi, 1974). DMFT treats the local dynamics exactly, but it cannot account for the spatial correlations being important in real finite-dimensional systems. Nevertheless DMFT allows one to address the intermediate coupling and adiabaticity regimes in the absorption not covered by the standard small polaron theory, where qualitatively new features arise. In particular, the optical absorption exhibits a reentrant behavior, switching from weak-coupling-like to polaronic-like upon increasing the temperature, and sharp peaks with a nonmonotonic temperature dependence emerge at characteristic phonon frequencies (Fratini and Ciuchi, 2006).

Earlier ED studies of the lattice polaron absorption were limited to small 2 to 10-site 1D and 2D clusters in the Holstein model (Alexandrov et al. 1994b; Capone et al. 1, 1997; Fehske et al. ., 1997; Wellein and Fehske,, 1998). The optical absorption occurs as energy is transferred between the electromagnetic field and the phonons via the charge carriers. The vibration energy must be capable of being dissipated. Hence, using ED one has to introduce some continuous density of phonon states, or a phonon lifetime, which makes MIR absorption to be finite (Alexandrov et al. 1994b). As a result one obtains a fair agreement between ED absorption spectra and the analytical results, Eq.(221), in the strong coupling limit as far as a smooth part of $\omega$ dependence is concerned. The MIR conductivity occurs much more asymmetric in the intermediate coupling region than in the strong coupling regime, 
and it shows an additional oscillating superstructure corresponding to a different spectral weight of the states with a different number of virtual phonons in the polaron cloud.

More recent ED, VED and a kernel polynomial method (KPM) (for a review of KPM

see (Weiße et al. , 2006)) allowed for numerical calculations of lattice polaron properties in the Holstein model in the whole parameter range on fairly large systems (Barisic, 2004; El Shawish et al. , 2003; (Schubert et al. , 2005). The main signature of optical conductivity for intermediate-to-strong EP coupling in the adiabatic regime (Fehske and Trugman , 2007) is that the spectrum is strongly asymmetric, which is also characteristic for rather large polarons (III.F), as observed in cuprate superconductors (Mihailović et al. , 1990), perovskite tungsten bronzes (Ruscher et al. ,2008) and many other doped insulators. Importantly, the weaker decay at the high-energy side meets the experimental findings for many polaronic materials like $\mathrm{TiO}_{2}$ (Kudinov et al. , 1969) even better than standard small-polaron theory. At larger EPI a more pronounced and symmetric maximum appears in the low-temperature optical response. When the phonon frequency becomes comparable to the electron transfer amplitude different absorption bands appear, which can be classified according to the number of phonons involved in the optical transition (Fehske and Trugman, 2007).

\section{Spectral function of discrete strong-coupling polarons}

The polaron problem has the exact solution, Eq.(172), in the extreme infinite-coupling limit, $\lambda=\infty$, for any EPI conserving the on-site occupation numbers of electrons. For a finite but strong coupling $1 / \lambda$ perturbation expansion is applied. Importantly, the analytical perturbation theory becomes practically exact in a wider range of the adiabatic parameter and of the coupling constant for the long-range Fröhlich interaction (IV.E.3).

Keeping this in mind, let us calculate the polaron spectral function in the first order in $1 / \lambda$ (Alexandrov and Ranninger, 1992; Alexandrov and Sricheewin , 2000). We can neglect $H_{p-p h}$ in the first-order if $1 / \lambda \ll 1$. To understand spectral properties of a single polaron we also neglect the polaron-polaron interaction. Then the energy levels are

$$
E_{\tilde{m}}=\sum_{\mathbf{k}} \xi_{\mathbf{k}} n_{\mathbf{k}}+\sum_{\mathbf{q}} \omega_{\mathbf{q}}\left[n_{\mathbf{q}}+1 / 2\right]
$$

where $\xi_{\mathbf{k}}$ is the small polaron band dispersion with respect to the chemical potential, and the transformed eigenstates $|\tilde{m}\rangle$ are sorted by the polaron Bloch-state occupation numbers, 
$n_{\mathbf{k}}=0,1$, and the phonon occupation numbers, $n_{\mathbf{q}}=0,1,2, \ldots, \infty$.

The spectral function of any system described by quantum numbers $m, n$ with the eigenvalues $E_{n}, E_{m}$ is defined as

$$
A(\mathbf{k}, \omega) \equiv \pi\left(1+e^{-\omega / T}\right) e^{\Omega / T} \sum_{n, m} e^{-E_{n} / T}\left|\left\langle n\left|c_{\mathbf{k}}\right| m\right\rangle\right|^{2} \delta\left(\omega_{n m}+\omega\right)
$$

It is real and positive, $A(\mathbf{k}, \omega)>0$, and obeys the sum rule

$$
\frac{1}{\pi} \int_{-\infty}^{\infty} d \omega A(\mathbf{k}, \omega)=1
$$

The matrix elements of the electron operators can be written as

$$
\left\langle n\left|c_{\mathbf{k}}\right| m\right\rangle=\frac{1}{\sqrt{N}} \sum_{\mathbf{m}} e^{-i \mathbf{k} \cdot \mathbf{m}}\left\langle\tilde{n}\left|c_{i} \hat{X}_{i}\right| \tilde{m}\right\rangle
$$

using the Wannier representation and the Lang-Firsov transformation. Now, applying the Fourier transform of the $\delta$-function, the spectral function is expressed as

$$
\begin{aligned}
A(\mathbf{k}, \omega)= & \frac{1}{2} \int_{-\infty}^{\infty} d t e^{i \omega t} \frac{1}{N} \sum_{\mathbf{m}, \mathbf{n}} e^{i \mathbf{k} \cdot(\mathbf{n}-\mathbf{m})} \times \\
& \left\{\left\langle\left\langle c_{i}(t) \hat{X}_{i}(t) c_{j}^{\dagger} \hat{X}_{j}^{\dagger}\right\rangle\right\rangle+\left\langle\left\langle c_{j}^{\dagger} \hat{X}_{j}^{\dagger} c_{i}(t) \hat{X}_{i}(t)\right\rangle\right\rangle\right\} .
\end{aligned}
$$

Here the quantum and statistical averages are performed for independent polarons and phonons, therefore $\left\langle\left\langle c_{i}(t) \hat{X}_{i}(t) \hat{X}_{j}^{\dagger} c_{i}^{\dagger}\right\rangle\right\rangle=\left\langle\left\langle c_{i}(t) c_{j}^{\dagger}\right\rangle\right\rangle\left\langle\left\langle\hat{X}_{i}(t) \hat{X}_{j}^{\dagger}\right\rangle\right\rangle$. The Heisenberg free-polaron operator evolves with time as $c_{\mathbf{k}}(t)=c_{\mathbf{k}} \exp \left(-i \xi_{\mathbf{k}} t\right)$, so that

$$
\begin{aligned}
\left\langle\left\langle c_{i}(t) c_{i}^{\dagger}\right\rangle\right\rangle= & \frac{1}{N} \sum_{\mathbf{k}^{\prime}, \mathbf{k}^{\prime \prime}} e^{i\left(\mathbf{k}^{\prime} \cdot \mathbf{m}-\mathbf{k}^{\prime \prime} \cdot \mathbf{n}\right)}\left\langle\left\langle c_{\mathbf{k}^{\prime}}(t) c_{\mathbf{k}^{\prime \prime}}^{\dagger}\right\rangle\right\rangle= \\
& \frac{1}{N} \sum_{\mathbf{k}^{\prime}}\left[1-\bar{n}\left(\mathbf{k}^{\prime}\right)\right] e^{i \mathbf{k}^{\prime} \cdot(\mathbf{m}-\mathbf{n})-i \xi_{\mathbf{k}^{\prime}} t}, \\
\left\langle\left\langle c_{i}^{\dagger} c_{i}(t)\right\rangle\right\rangle= & \frac{1}{N} \sum_{\mathbf{k}^{\prime}} \bar{n}\left(\mathbf{k}^{\prime}\right) e^{i \mathbf{k}^{\prime} \cdot(\mathbf{m}-\mathbf{n})-i \xi_{\mathbf{k}^{\prime}} t}
\end{aligned}
$$

where $\bar{n}(\mathbf{k})=\left[1+\exp \xi_{\mathbf{k}} / T\right]^{-1}$ is the Fermi-Dirac distribution function of polarons. The Heisenberg free-phonon operator evolves in a similar way, $d_{\mathbf{q}}(t)=d_{\mathbf{q}} \exp \left(-i \omega_{\mathbf{q}} t\right)$, so that

$$
\left\langle\left\langle\hat{X}_{i}(t) \hat{X}_{j}^{\dagger}\right\rangle\right\rangle=\prod_{\mathbf{q}}\left\langle\left\langle\exp \left[u_{i}(\mathbf{q}, t) d_{\mathbf{q}}-H . c .\right] \exp \left[-u_{j}(\mathbf{q}) d_{\mathbf{q}}-H . c .\right]\right\rangle\right\rangle,
$$

where $u_{i, j}(\mathbf{q}, t)=u_{i, j}(\mathbf{q}) \exp \left(-i \omega_{\mathbf{q}} t\right)$. This average is calculated using the operator identity, as in Eq.(177),

$$
\left\langle\left\langle\hat{X}_{i}(t) \hat{X}_{j}^{\dagger}\right\rangle\right\rangle=\exp \left\{-\frac{1}{2 N} \sum_{\mathbf{q}}|\gamma(\mathbf{q})|^{2} f_{\mathbf{q}}(\mathbf{m}-\mathbf{n}, t)\right\}
$$


where

$$
f_{\mathbf{q}}(\mathbf{m}, t)=\left[1-\cos (\mathbf{q} \cdot \mathbf{m}) \cos \left(\omega_{\mathbf{q}} t\right)\right] \operatorname{coth} \frac{\omega_{\mathbf{q}}}{2 T}+i \cos (\mathbf{q} \cdot \mathbf{m}) \sin \left(\omega_{\mathbf{q}} t\right) .
$$

Here we used the symmetry of $\gamma(-\mathbf{q})=\gamma(\mathbf{q})$, so that terms containing $\sin (\mathbf{q} \cdot \mathbf{m})$ have disappeared.

To proceed with the analytical results we consider low temperatures, $T \ll \omega_{\mathbf{q}}$, when $\operatorname{coth}\left(\omega_{\mathbf{q}} / 2 T\right) \approx 1$. Expanding the exponent in Eq.(230) and performing summation over $\mathbf{m}, \mathbf{n}, \mathbf{k}^{\prime}$ and integration over time we arrive at (Alexandrov and Sricheewin, 2000)

$$
A(\mathbf{k}, \omega)=\sum_{l=0}^{\infty}\left[A_{l}^{(-)}(\mathbf{k}, \omega)+A_{l}^{(+)}(\mathbf{k}, \omega)\right],
$$

where

$$
\begin{aligned}
& A_{l}^{(-)}(\mathbf{k}, \omega)=\pi Z \sum_{\mathbf{q}_{1}, \ldots \mathbf{q}_{l}} \frac{\prod_{r=1}^{l}\left|\gamma\left(\mathbf{q}_{r}\right)\right|^{2}}{(2 N)^{l} l !} \times \\
& {\left[1-\bar{n}\left(\mathbf{k}-\sum_{r=1}^{l} \mathbf{q}_{r}\right)\right] \delta\left(\omega-\sum_{r=1}^{l} \omega_{\mathbf{q}_{r}}-\xi_{\mathbf{k}-\sum_{r=1}^{l} \mathbf{q}_{r}}\right),} \\
& A_{l}^{(+)}(\mathbf{k}, \omega)=\pi Z \sum_{\mathbf{q}_{1}, \ldots \mathbf{q}_{l}} \frac{\prod_{r=1}^{l}\left|\gamma\left(\mathbf{q}_{r}\right)\right|^{2}}{(2 N)^{l} l !} \times \\
& \bar{n}\left(\mathbf{k}+\sum_{r=1}^{l} \mathbf{q}_{r}\right) \delta\left(\omega+\sum_{r=1}^{l} \omega_{\mathbf{q}_{r}}-\xi_{\mathbf{k}+\sum_{r=1}^{l}} \mathbf{q}_{r}\right),
\end{aligned}
$$

and $Z=\exp \left(-(2 N)^{-1} \sum_{\mathbf{q}}|\gamma(\mathbf{q})|^{2}\right)$.

Clearly Eq.(232) is in the form of the perturbative multi-phonon expansion. Each contribution $A_{l}^{( \pm)}(\mathbf{k}, \omega)$ to the spectral function describes the transition from the initial state $\mathbf{k}$ of the polaron band to the final state $\mathbf{k} \pm \sum_{r=1}^{l} \mathbf{q}_{r}$ with the emission (or absorption) of $l$ phonons.

The $1 / \lambda$ expansion result, Eq.(232), is different from the conventional spectral function of metallic electrons coupled to phonons in the Migdal-Eliashberg theory (Eliashberg, 1960; Migdal , 1958). There is no imaginary part of the self-energy since the exponentially small (at low temperatures) polaronic damping, Eq.(187), is neglected. Instead EPI leads to the coherent dressing of electrons by phonons, and phonon "side-bands". The spectral function of the polaronic carriers comprises two different parts. The first $(l=0) \mathbf{k}$-dependent coherent term arises from the polaron band tunnelling,

$$
A_{c o h}(\mathbf{k}, \omega)=\left[A_{0}^{(-)}(\mathbf{k}, \omega)+A_{0}^{(+)}(\mathbf{k}, \omega)\right]=\pi Z \delta\left(\omega-\xi_{\mathbf{k}}\right) .
$$


The spectral weight of the coherent part is suppressed as $Z \ll 1$. However in the case of the Fröhlich interaction the effective mass is less enhanced, since the band narrowing factor $Z^{\prime}$, Eq.(207), in $\xi_{\mathbf{k}}=Z^{\prime} E_{\mathbf{k}}-\mu$ is large compared with $Z$ (Alexandrov and Sricheewin, 2000).

The second incoherent part $A_{\text {incoh }}(\mathbf{k}, \omega)$ comprises all the terms with $l \geq 1$. It describes excitations accompanied by emission and absorption of phonons. We notice that its spectral density spreads over a wide energy range of about twice the polaron level shift $E_{p}$, which might be larger than the unrenormalised bandwidth $2 D$ in the rigid lattice without phonons. On the contrary, the coherent part shows a dispersion only in the energy window of the order of the polaron bandwidth, $w=Z^{\prime} D$. It is interesting that there is some $\mathbf{k}$ dependence of the incoherent background as well, if the EPI matrix element and/or phonon frequencies depend on q. Only in the Holstein model with the short-range dispersionless e-ph interaction $\gamma(\mathbf{q})=\gamma_{0}$ and $\omega_{\mathbf{q}}=\omega_{0}$ the incoherent part is momentum independent (Mahan, 1990),

$$
\begin{aligned}
A_{\text {incoh }}(\mathbf{k}, \omega)= & \pi \frac{Z}{N} \sum_{l=1}^{\infty} \frac{\gamma_{0}^{2 l}}{2^{l} l !} \times \\
& \sum_{\mathbf{k}^{\prime}}\left\{\left[1-\bar{n}\left(\mathbf{k}^{\prime}\right)\right] \delta\left(\omega-l \omega_{0}-\xi_{\mathbf{k}^{\prime}}\right)+\bar{n}\left(\mathbf{k}^{\prime}\right) \delta\left(\omega+l \omega_{0}-\xi_{\mathbf{k}^{\prime}}\right)\right\} .
\end{aligned}
$$

As soon as we know the spectral function, different Green's functions (GF) are readily obtained using their analytical properties. For example, the temperature GF is given by the integral

$$
\mathcal{G}\left(\mathbf{k}, \omega_{k}\right)=\frac{1}{\pi} \int_{-\infty}^{\infty} d \omega^{\prime} \frac{A\left(\mathbf{k}, \omega^{\prime}\right)}{i \omega_{k}-\omega^{\prime}} .
$$

where $\omega_{k}=\pi T(2 k+1), k=0, \pm 1, \pm 2, \ldots$. Calculating the integral we find in the Holstein model (Alexandrov and Ranninger, 1992)

$$
\mathcal{G}\left(\mathbf{k}, \omega_{n}\right)=\frac{Z}{i \omega_{n}-\xi_{\mathbf{k}}}+\frac{Z}{N} \sum_{l=1}^{\infty} \frac{\gamma_{0}^{2 l}}{2^{l} l !} \sum_{\mathbf{k}^{\prime}}\left\{\frac{1-\bar{n}\left(\mathbf{k}^{\prime}\right)}{i \omega_{n}-l \omega_{0}-\xi_{\mathbf{k}^{\prime}}}+\frac{\bar{n}\left(\mathbf{k}^{\prime}\right)}{i \omega_{n}+l \omega_{0}-\xi_{\mathbf{k}^{\prime}}}\right\} .
$$

Here the first term describes the coherent tunnelling in the narrow polaron band while the second $\mathbf{k}$-independent sum describes the phonon-side bands.

\section{Spectral function of discrete all-coupling polarons}

The spectral function, Eq.(238), satisfies the major sum rule, Eq.(224). However the higher-momentum integrals, $\int_{\infty}^{\infty} d \omega \omega^{p} A(\mathbf{k}, \omega)$ with $p>0$, calculated using Eq.(238), differ from the exact values (Kornilovitch , 2002) by an amount proportional to $1 / \lambda$. The difference 
is due to a partial "undressing" of high-energy excitations in the side-bands, which is beyond the first order $1 / \lambda$ expansion. A rather accurate Green's function of the Holstein polaron has been recently obtained by summing all the diagrams, but with each diagram averaged over its free propagators' momenta (Berciu, 2006a, $\underline{b}$ ). The resulting Green's function satisfies exactly the first six spectral weight sum rules. All higher sum rules are satisfied with great accuracy, becoming asymptotically exact for coupling both much larger and much smaller than the free particle bandwidth.

The spectral properties of the Holstein model in a wider parameter range have been studied numerically using ED (see (Alexandrov et al. , 1994b; Capone et al. , 1997; El Shawish et al. ,2003; Fehske et al. , 1997; Fehske and Trugman , 2007; Looset al. , 2006; Ranninger and Thibblin, 1992; Schubert et al. , 2005; Wellein and Fehske, 1998), and references therein). At the weak EPI, the electronic spectrum of $1 \mathrm{D}$ Holstein model is nearly unaffected for energies below the phonon emission threshold (Fehske and Trugman, 2007). Hence, the renormalised dispersion $\epsilon_{k}$ nearly coincides with the tight-binding cosine band (shifted $\propto E_{p}$ ) up to some $k_{X}$, where the phonon energy intersects the bare electron band with the familiar flattening effect (IV.F). Reaching the intermediate EPI (polaron crossover) regime a coherent band separates from the rest of the spectrum $\left[k_{X} \rightarrow \pi\right]$. At the same time its spectral weight becomes smaller and will be transferred to the incoherent part, where several sub-bands emerge. In the strong-coupling case the coherent quasi-particle absorption band becomes extremely narrow and its bandwidth approaches the strong-coupling result. The incoherent part of the spectrum carries most of the spectral weight consisting of a sequence of sub-bands separated in energy by $\omega_{0}$, in agreement with the analytical results.

Effects of the finite-range EPI on the spectral properties of lattice polarons have been studied numerically by (Fehske et al. , 2002) using exact Lanczos diagonalisation method in the framework of the 1D model of (Alexandrov and Kornilovitch, 1999). The polaron band-structure has been calculated in agreement with the analytical and CTQMC results IV.E.3. The optical absorption of lattice polarons with a finite-range (Fröhlich-type) EPI has been found similar to the continuous-polaron absorption (III.F) for all EPI strengths. The polaron features due to EPIs with different coupling ranges were also investigated in the framework of the variational approach (Perroni et al., 2005). The ground-state energy, the spectral weight, the average kinetic energy, the mean number of phonons, and the electron-lattice correlation function were calculated for the system with coupling to local 
and nearest-neighbor lattice displacements and compared with the long-range case. As in (Alexandrov and Sricheewin, 2000) a substantially different mass renormalisation compared with the coherent weight reduction, $Z \ll Z^{\prime}$, was found for the finite-range EPI.

\section{BIPOLARON}

\section{A. Polaron-polaron interaction}

Polarons interact with each other, cf. Eq.(171) for small polarons. The range of the deformation surrounding the Fröhlich polarons is quite large, and their deformation fields are overlapped at finite density. Taking into account both the long-range attraction of polarons owing to the lattice deformations and their direct Coulomb repulsion, the residual long-range interaction turns out rather weak and repulsive in ionic crystals (Alexandrov and Mott, 1995). In the long-wave limit $(q \ll \pi / a)$ the Fröhlich EPI dominates in the attractive part, but polarons repel each other at large distances, $|\mathbf{m}-\mathbf{n}| \gg a$,

$$
v(\mathbf{m}-\mathbf{n})=\frac{e^{2}}{\varepsilon_{0}|\mathbf{m}-\mathbf{n}|}>0 .
$$

The Fröhlich EPI nearly nullifies the bare Coulomb repulsion, if $\varepsilon_{0} \gg 1$, but cannot overscreen it at large distances.

Considering the polaron-phonon interaction in the multi-polaron system we have to take into account the dynamic properties of the polaron response function (Alexandrov, 2000). One may erroneously believe that the long-range Fröhlich EPI becomes a short-range (Holstein) one due to screening of ionic potentials by heavy polaronic carriers. In fact, as distinct from large polarons, small polarons cannot screen high-frequency optical vibrations because their renormalised plasma frequency is comparable with or even less than the phonon frequency in the strong-coupling regime (Alexandrov, 1992a). The small-polaron plasma frequency is rather low due to the large static dielectric constant, $\varepsilon_{0} \gg 1$, in ionic lattices and the enhanced polaron mass $m^{*} \gg m$. If $\omega_{0}>\omega_{p}$, the singular behaviour of the Fröhlich EPI, $\gamma(\mathbf{q}) \sim 1 / q$, is unaffected by screening. Polarons are too slow to screen high-frequency crystal field oscillations. As a result, EPI with high-frequency optical phonons in ionic solids remains unscreened at any density of polarons.

Another important issue is a possibility of the Wigner crystallization of polarons. Because the net long-range interaction is relatively weak, a relevant dimensionless parameter $r_{s}=$ 
$m^{*} a e^{2} / \varepsilon_{0}(4 \pi n / 3)^{1 / 3}$ is not very large in ionic semiconductors. The Wigner crystallization appears around $r_{s} \simeq 100$ or larger, which corresponds to the atomic density of polarons $n \leq 10^{-6}$ with $\varepsilon_{0}=30$ and $m^{*}=5 \mathrm{~m}$. This estimate tells us that sufficiently mobile small polarons can be in a liquid state (Alexandrov, 2000) at substantial doping levels, however they can be crystallised at low doping (see III.H).

When the short-range deformation and molecular-type (i.e. Holstein) EPIs are added to the Fröhlich interaction, two polarons attract each other at a short distance of about the lattice constant. Then, owing to a narrow band, two lattice polarons easily form a local tightly bound state, i.e. a small bipolaron (Alexandrov and Ranninger, 1981a, b; ;nderson, 1975; Aubry, 1993; Beni et al. , 1974; Street and Mott, 1975). One can estimate the coupling constant $\lambda$ and the adiabatic ratio $\omega_{0} / t$, at which the small bipolaron "instability" occurs (Alexandrov, 2000). The characteristic attractive potential is $|v|=D /\left(\lambda-\mu_{c}\right)$, where $\mu_{c}$ is the dimensionless Coulomb repulsion, and $\lambda$ includes the interaction with all phonon modes. The radius of the potential is about $a$. In three dimensions a bound state of two attractive particles appears, if $|v| \geq \pi^{2} / 8 m^{*} a^{2}$. Substituting the polaron mass, $m^{*}=\left[2 a^{2} t\right]^{-1} \exp \left(\gamma \lambda D / \omega_{0}\right)$, we find

$$
\frac{t}{\omega_{0}} \leq(\gamma z \lambda)^{-1} \ln \left[\frac{\pi^{2}}{4 z\left(\lambda-\mu_{c}\right)}\right] .
$$

As a result, small bipolarons form at $\lambda \geq \mu_{c}+\pi^{2} / 4 z$, which is almost independent of the adiabatic ratio.

The formation of small bipolarons is closely related to a negative $U$-center model (Street and Mott, 1975). Starting from initial works by Eagles (Eagles, 1969) and Legget (Legget, 1980) this model received particular attention in connection with high-temperature superconductivity (Micnas et al. , 1990). However, in using the negative Hubbard $U$ model, one has to realize that this model, which predicts a smooth crossover from Cooper pairs to real-space pairs (Nozieres and Schmitt-Rink, 1985), cannot account for the polaronbipolaron crossover with the increasing EPI. The essential effect of the polaron bandnarrowing, which is responsible for high critical temperatures in the model of polaronic superconductors (Alexandrov, 1983), is missing in the negative Hubbard $U$ model. 


\section{B. Holstein bipolaron}

The attractive energy of two small polarons is generally much larger than the polaron bandwidth, which allows for a consistent treatment of small bipolarons (Alexandrov, 1983; Alexandrov and Ranninger, 1981a, b). Under this condition the hopping term in the transformed Hamiltonian $\tilde{H}$ is a small perturbation of the ground state of immobile bipolarons and free phonons,

$$
\tilde{H}=H_{0}+H_{\text {pert }}
$$

where

$$
H_{0}=\frac{1}{2} \sum_{i, j} v_{i j} c_{i}^{\dagger} c_{j}^{\dagger} c_{j} c_{i}+\sum_{\mathbf{q}, \nu} \omega_{\mathbf{q} \nu}\left[d_{\mathbf{q} \nu}^{\dagger} d_{\mathbf{q} \nu}+1 / 2\right]
$$

and

$$
H_{\text {pert }}=\sum_{i, j} \hat{\sigma}_{i j} c_{i}^{\dagger} c_{j}
$$

Let us first discuss the dynamics of onsite bipolarons, which are the ground state of the system with the Holstein non-dispersive EPI (Alexandrov and Ranninger), 1981a; Anderson, 1975; Aubry, 1993, 1995; Beni et al. 1, 1974; Street and Mott, 1975). The onsite bipolaron is formed if $2 E_{p}>U$, where $U$ is the onsite Coulomb correlation energy (the Hubbard $U$ ). The intersite polaron-polaron interaction, Eq.(171), is just the Coulomb repulsion since the phonon mediated attraction between two polarons on different sites is zero in the Holstein model. Two or more onsite bipolarons as well as three or more polarons cannot occupy the same site because of the Pauli exclusion principle. Hence, bipolarons repel single polarons and each other. Their binding energy, $\Delta=2 E_{p}-U$, is larger than the polaron half-bandwidth, $\Delta \gg w$, so that there are no unbound polarons in the ground state. $H_{\text {pert }}$, Eq.(243), destroys bipolarons in the first order. Hence it has no diagonal matrix elements. Then the bipolaron dynamics is described by the use of another canonical transformation $\exp \left(S_{2}\right)$ (Alexandrov and Ranninger, 1981a), which eliminates the first order of $H_{\text {pert }}$,

$$
\left(S_{2}\right)_{f p}=\sum_{i, j} \frac{\left\langle f\left|\hat{\sigma}_{i j} c_{i}^{\dagger} c_{j}\right| p\right\rangle}{E_{f}-E_{p}} .
$$

Here $E_{f, p}$ and $|f\rangle,|p\rangle$ are the energy levels and the eigenstates of $H_{0}$. Neglecting the terms of the order higher than $(w / \Delta)^{2}$ one obtains

$$
\left(H_{b}\right)_{f f^{\prime}} \equiv\left(e^{S_{2}} \tilde{H} e^{-S_{2}}\right)_{f f^{\prime}},
$$




$$
\begin{aligned}
\left(H_{b}\right)_{f f^{\prime}} \approx & \left(H_{0}\right)_{f f^{\prime}}-\frac{1}{2} \sum_{\nu} \sum_{i \neq i^{\prime}, j \neq j^{\prime}}\left\langle f\left|\hat{\sigma}_{i i^{\prime}} c_{i}^{\dagger} c_{i^{\prime}}\right| p\right\rangle\left\langle p\left|\hat{\sigma}_{j j^{\prime}} c_{j}^{\dagger} c_{j^{\prime}}\right| f^{\prime}\right\rangle \times \\
& \left(\frac{1}{E_{p}-E_{f^{\prime}}}+\frac{1}{E_{p}-E_{f}}\right) .
\end{aligned}
$$

The bipolaronic Hamiltonian $H_{b}$ is defined in the subspace $|f\rangle,\left|f^{\prime}\right\rangle$ with no single (unbound) polarons. On the other hand, the intermediate bra $\langle p|$ and ket $|p\rangle$ refer to configurations involving two unpaired polarons and any number of phonons. Hence we have

$$
E_{p}-E_{f}=\Delta+\sum_{\mathbf{q}, \nu} \omega_{\mathbf{q} \nu}\left(n_{\mathbf{q} \nu}^{p}-n_{\mathbf{q} \nu}^{f}\right)
$$

where $n_{\mathbf{q} \nu}^{f, p}$ are the phonon occupation numbers $(0,1,2,3 \ldots \infty)$.

The lowest eigenstates of $H_{b}$ are in the subspace, which has only doubly occupied $c_{\mathbf{m} s}^{\dagger} c_{\mathbf{m} s^{\prime}}^{\dagger}|0\rangle$ or empty $|0\rangle$ sites. On-site bipolaron tunnelling is a two-step transition. It takes place via a single polaron tunneling to a neighbouring site. The subsequent tunnelling of its "partner" to the same site restores the initial energy state of the system. There are no real phonons emitted or absorbed because the (bi)polaron band is narrow. Hence we can average $H_{b}$ with respect to phonons,

$$
\begin{aligned}
H_{b}= & H_{0}-i \sum_{\mathbf{m} \neq \mathbf{m}^{\prime}, s} \sum_{\mathbf{n} \neq \mathbf{n}^{\prime}, s^{\prime}} t\left(\mathbf{m}-\mathbf{m}^{\prime}\right) t\left(\mathbf{n}-\mathbf{n}^{\prime}\right) \times \\
& c_{\mathbf{m} s}^{\dagger} c_{\mathbf{m}^{\prime} s} c_{\mathbf{n} s^{\prime}}^{\dagger} c_{\mathbf{n}^{\prime} s^{\prime}} \int_{0}^{\infty} d t e^{-i \Delta t} \Phi_{\mathbf{m}^{\prime}}^{\mathbf{n n}^{\prime}}(t) .
\end{aligned}
$$

Here $\Phi_{\mathbf{m m}^{\prime}}^{\mathbf{n n}^{\prime}}(t)$ is a multiphonon correlator,

$$
\Phi_{\mathbf{m m}^{\prime}}^{\mathbf{n n}^{\prime}}(t) \equiv\left\langle\left\langle\hat{X}_{i}^{\dagger}(t) \hat{X}_{i^{\prime}}(t) \hat{X}_{j}^{\dagger} \hat{X}_{j^{\prime}}\right\rangle\right\rangle
$$

which can be readily calculated as (Alexandrov, 1992a)

$$
\begin{aligned}
\Phi_{\mathbf{m m}^{\prime}}^{\mathbf{n n}^{\prime}}(t)= & e^{-g^{2}\left(\mathbf{m}-\mathbf{m}^{\prime}\right)} e^{-g^{2}\left(\mathbf{n}-\mathbf{n}^{\prime}\right)} \times \\
& \exp \left\{\frac{1}{2 N} \sum_{\mathbf{q}, \nu}|\gamma(\mathbf{q}, \nu)|^{2} F_{\mathbf{q}}\left(\mathbf{m}, \mathbf{m}^{\prime}, \mathbf{n}, \mathbf{n}^{\prime}\right) \frac{\cosh \left[\omega_{\mathbf{q} \nu}\left(\frac{1}{2 T}-i t\right)\right]}{\sinh \left[\frac{\omega_{\mathbf{q} \nu}}{2 T}\right]}\right\},
\end{aligned}
$$

where $F_{\mathbf{q}}\left(\mathbf{m}, \mathbf{m}^{\prime}, \mathbf{n}, \mathbf{n}^{\prime}\right)=\cos \left[\mathbf{q} \cdot\left(\mathbf{n}^{\prime}-\mathbf{m}\right)\right]+\cos \left[\mathbf{q} \cdot\left(\mathbf{n}-\mathbf{m}^{\prime}\right)\right]-\cos \left[\mathbf{q} \cdot\left(\mathbf{n}^{\prime}-\mathbf{m}^{\prime}\right)\right]-\cos [\mathbf{q} \cdot(\mathbf{n}-$ $\mathbf{m})]$.

Taking into account that there are only bipolarons in the subspace, where $H_{b}$ operates, one can rewrite the Hamiltonian in terms of the creation $b_{\mathbf{m}}^{\dagger}=c_{\mathbf{m} \uparrow}^{\dagger} c_{\mathbf{m} \downarrow}^{\dagger}$ and annihilation 
$b_{\mathbf{m}}=c_{\mathbf{m} \downarrow} c_{\mathbf{m} \uparrow}$ operators of singlet pairs (Alexandrov and Ranninger, 1981a):

$$
\begin{aligned}
H_{b}= & -\sum_{\mathbf{m}}\left[\Delta+\frac{1}{2} \sum_{\mathbf{m}^{\prime}} v^{(2)}\left(\mathbf{m}-\mathbf{m}^{\prime}\right)\right] n_{\mathbf{m}}+ \\
& \sum_{\mathbf{m} \neq \mathbf{m}^{\prime}}\left[t_{b}\left(\mathbf{m}-\mathbf{m}^{\prime}\right) b_{\mathbf{m}}^{\dagger} b_{\mathbf{m}^{\prime}}+\frac{1}{2} \bar{v}\left(\mathbf{m}-\mathbf{m}^{\prime}\right) n_{\mathbf{m}} n_{\mathbf{m}^{\prime}}\right] .
\end{aligned}
$$

Here $n_{\mathbf{m}}=b_{\mathbf{m}}^{\dagger} b_{\mathbf{m}}$ is the bipolaron site-occupation operator, $\bar{v}\left(\mathbf{m}-\mathbf{m}^{\prime}\right)=4 v\left(\mathbf{m}-\mathbf{m}^{\prime}\right)+$ $v^{(2)}\left(\mathbf{m}-\mathbf{m}^{\prime}\right)$ is the bipolaron-bipolaron interaction including the direct polaron-polaron interaction $v\left(\mathbf{m}-\mathbf{m}^{\prime}\right)$ and a repulsive correlation energy,

$$
v^{(2)}\left(\mathbf{m}-\mathbf{m}^{\prime}\right)=2 i \int_{0}^{\infty} d \tau e^{-i \Delta \tau} \Phi_{\mathbf{m m}^{\prime}}^{\mathbf{m}^{\prime} \mathbf{m}}(\tau) .
$$

This additional repulsion appears since a virtual hop of one of two polarons of the pair is forbidden, if the neighbouring site is occupied by another pair. The bipolaron transfer integral, $t_{b}$ is of the second order in $t(\mathbf{m})$

$$
t_{b}\left(\mathbf{m}-\mathbf{m}^{\prime}\right)=-2 i t^{2}\left(\mathbf{m}-\mathbf{m}^{\prime}\right) \int_{0}^{\infty} d \tau e^{-i \Delta \tau} \Phi_{\mathbf{m m}^{\prime}}^{\mathbf{m m}^{\prime}}(\tau) .
$$

The multiphonon correlator is simplified for dispersionless phonons at $T \ll \omega_{0}$ as

$$
\begin{aligned}
& \Phi_{\mathbf{m}^{\prime} \mathbf{m}^{\prime}}^{\mathbf{m}^{\prime}}(t)=e^{-2 g^{2}\left(\mathbf{m}-\mathbf{m}^{\prime}\right)} \exp \left[-2 g^{2}\left(\mathbf{m}-\mathbf{m}^{\prime}\right) e^{-i \omega_{0} t}\right] \\
& \Phi_{\mathbf{m}^{\prime} \mathbf{m}^{\prime}}^{\mathbf{m}^{\prime}}(t)=e^{-2 g^{2}\left(\mathbf{m}-\mathbf{m}^{\prime}\right)} \exp \left[2 g^{2}\left(\mathbf{m}-\mathbf{m}^{\prime}\right) e^{-i \omega_{0} t}\right]
\end{aligned}
$$

which yields (Alexandrov and Kabanov, 1986)

$$
t(\mathbf{m})=-\frac{2 t^{2}(\mathbf{m})}{\Delta} e^{-2 g^{2}(\mathbf{m})} \sum_{l=0}^{\infty} \frac{\left[-2 g^{2}(\mathbf{m})\right]^{l}}{l !\left(1+l \omega_{0} / \Delta\right)}
$$

and

$$
v^{(2)}(\mathbf{m})=\frac{2 t^{2}(\mathbf{m})}{\Delta} e^{-2 g^{2}(\mathbf{m})} \sum_{l=0}^{\infty} \frac{\left[2 g^{2}(\mathbf{m})\right]^{l}}{l !\left(1+l \omega_{0} / \Delta\right)} .
$$

When $\Delta \ll \omega_{0}$, we can keep the first term only with $l=0$ in the bipolaron hopping integral, Eq.(252). In this case the bipolaron half-bandwidth $z t(\mathbf{a})$ is of the order of $2 w^{2} /(z \Delta)$. However, if the bipolaron binding energy is large, $\Delta \gg \omega_{0}$, the bipolaron bandwidth dramatically decreases proportionally to $e^{-4 g^{2}}$ in the limit $\Delta \rightarrow \infty$. This limit is not realistic since $\Delta=2 E_{p}-V_{c}<2 g^{2} \omega_{0}$. In a more realistic regime, $\omega_{0}<\Delta<2 g^{2} \omega_{0}$, Eq.(252) yields (Alexandrov and Kabanov, 1986)

$$
t_{b}(\mathbf{m}) \approx \frac{2 \sqrt{2 \pi} t^{2}(\mathbf{m})}{\sqrt{\omega_{0} \Delta}} \exp \left[-2 g^{2}-\frac{\Delta}{\omega_{0}}\left(1+\ln \frac{2 g^{2}(\mathbf{m}) \omega_{0}}{\Delta}\right)\right] .
$$


On the contrary, the bipolaron-bipolaron repulsion, Eq.(254), has no small exponent in the limit $\Delta \rightarrow \infty, v^{(2)} \propto D^{2} / \Delta$. Together with the direct Coulomb repulsion the second order $v^{(2)}$ ensures stability of bipolarons against clustering.

Interestingly the high-temperature behavior of the bipolaron bandwidth is just opposite to that of the small polaron bandwidth. While the polaron band collapses with increasing temperature (IV.B.2), the bipolaron band becomes wider (Bryksin and Gol'tsev, 1988),

$$
t_{b}(\mathbf{m}) \propto \frac{1}{\sqrt{T}} \exp \left[-\frac{E_{p}+\Delta}{2 T}\right]
$$

for $T>\omega_{0}$.

The hopping conductivity of strong-coupling on-site bipolarons in the Holstein-Hubbard model (HHM) was found small compared with the hopping conductivity of thermally excited single polarons (Bryksin and Gol'tsev, 1988). However, as the frequency of the electric field increases, the dominant role in the optical absorption is gradually transferred to bipolarons at low temperatures. Like in the single-polaron case the bipolaron optical absorption can be estimated using the Franck-Condon principle which states that optical transitions take place without any change in the nuclear configuration. The corresponding analysis (Bryksin and Voloshin, 1984) shows that the absorption coefficient of light by the on-site bipolaron has three Gaussian peaks located at frequencies $\Omega=4 E_{a}, 8 E_{a}-U$ and $16 E_{a}$. The lowest peak corresponds to the absorption by single thermally excited polarons. The highest peak is due to the shakeoff of phonons without dissociation of the bipolaron while the main central peak is the absorption involving dissociation. A generalisation of the Franck-Condon principle for the optical absorption of intersite bipolarons with a finite-range EPI was given by (Alexandrov and Bratkovsky, 1999b).

The optical absorption and single-particle spectral functions of the bipolaron in 1D HHM in the whole range of parameters were calculated using ED on a 2-site cluster with two electrons (Alexandrov et al. , 1994b)), and more recently in (Hohenadler et al. , 2005) using the cluster perturbation theory (CPT). The latter allowed one to calculate the spectrum at continuous wave vectors and to find pronounced deviations (e.g. noncosine dispersions) of the bipolaron band structure from a simple tight-binding band due to an important contribution from the next-nearest-neighbour hoppings.

Treating phonons classically in the extreme adiabatic limit (Aubry, 1993, 1995, 2007; Proville and Aubry, 1998, 1999) found along with the onsite bipolaron (S0) also an 
anisotropic pair of polarons lying on two neighboring sites, i.e. the inter site bipolaron $(S 1)$. Such bipolarons were originally hypothesized in (Alexandrov, 1991) to explain the anomalous nuclear magnetic relaxation (NMR) in cuprate superconductors. The intersite bipolaron could take a form of a "quadrisinglet" $(Q S)$ in 2D HHM, where the electron density at the central site is 1 and " $1 / 4$ " on the four nearest neigbouring sites. In a certain region of $U$, where $Q S$ is the ground state, the double-well potential barrier, which usually pins polarons and bipolarons to the lattice, depresses to almost zero, so that adiabatic lattice bipolarons can be rather mobile (see (Aubry, 2007) for more details).

(Sil, 1999) investigated the stability of the bipolaronic phase in HHM using a modified Lang-Firsov variational transformation with on-site and nearest-neighbour lattice distortions. There is a critical on-site Hubbard repulsion $U_{c}$ below which the bipolaronic phases are stable for a fixed electron-phonon coupling. In the absence of on-site repulsion, bipolaronic phases are stable over the entire range of electron-phonon coupling for one dimension, whereas there is a critical electron-phonon coupling for formation of a stable bipolaron in two and three dimensions. Mobile $S 1$ bipolarons were found in 1D HHM using variational methods also in the non- and near-adiabatic regimes with dynamical quantum phonons (Bonča et al. ., 2000; La Magna and Pucci , 1997). The intersite bipolaron with a relatively small effective mass is stable in a wide region of the parameters of HHM due to both exchange and nonadiabaticity effects (La Magna and Pucci, 1997). Near the strong coupling limit the mobile $S 1$ bipolaron has an effective mass of the order of a single Holstein polaron mass, so that one should not rule out the possibility of a superconducting state of $S 1$ bipolarons with $s$ or $d$-wave symmetry in HHM (Bonča et al. , 2000). The recent diagrammatic Monte Carlo study (Macridin et al. , 2004) found $S 1$ bipolarons for large $U$ at intermediate and large EPI and established the phase diagram of 2D HHM, comprising large and small unbound polarons, $S 0$ and $S 1$ domains. Ref. (Macridin et al. , 2004) emphasised that the transition to the bound state and the properties of the bipolaron in HHM are very different from bound states in the attractive (negative $U$ ) Hubbard model without EPI (Robaszkiewicz et al. , 1981).

The two-dimensional many-body HHM was examined within a fluctuation-based effective cumulant approach by (Hakioglu and Zhuravlev, 1998) confirming that the numerical results on systems with finite degrees of freedom (IV.E) can be qualitatively extended to the systems with large degrees of freedom. When the electron-electron repulsion $U$ is dominant, the 
transition is to a Mott insulator; when EPI dominates, the transition is to a bipolaronic state. In the former case, the transition was found to be second order in contrast to the transition to the bipolaronic state, which is first order for larger values of $U$ (Koller et al. 2004b).

For a very strong electron-phonon coupling polarons become self-trapped on a single lattice site and bipolarons are on-site singlets. A finite bipolaron mass appears only in the second order of polaron hopping, Eq.(252), so that on-site bipolarons might be very heavy in the Holstein model with the short-range EPI. As a result the model led some authors to the conclusion that the formation of itinerant small polarons and bipolarons in real materials is unlikely (De Mello and Ranninger, 1997, 1998a), and high-temperature bipolaronic superconductivity is impossible (Anderson, 1997; Chakraverty et al. , 1998).

\section{Continuum Fröhlich bipolaron}

While polarons repel each other at large distances, two large polarons can be also bound into a large bipolaron by an exchange interaction even without any additional EPI but the Fröhlich one Adamowski, 1989; Bassani et al. . 1991; Hiramoto and Toyozawa , 1985; Kashirina et al. , 2005; Kochetov et al. , 1977; Mukhomorov, 1982; Suprun and Moizhes 1982; Verbist et al. , 1990, 1991; Vinetskii , 1961; Vinetskii and Giterman, 1957) (see also the reviews (Devreese, 1998; Smondyrev and Fomin, 1994)). Large bipolarons in the continuum limit are referred to as Fröhlich bipolarons. Besides the Fröhlich coupling constant, $\alpha$, the Fröhlich bipolaron energy depends also on the dimensionless parameter $U$, a measure for the strength of the Coulomb repulsion between the two electrons (Verbist et al. 1990, 1991), $U=\left(e^{2} / \varepsilon \omega_{0}\right) \sqrt{m \omega_{0}}$. In the discussion of bipolarons the ratio $\eta=\varepsilon / \varepsilon_{0}$ of the high-frequency (electronic) and static dielectric constants is important $(0 \leq \eta \leq 1)$. The following relation exists between $U$ and $\alpha$ : $U=\frac{\sqrt{2} \alpha}{1-\eta}$. Only values of $U$ satisfying the inequality $U \geq \sqrt{2} \alpha$ have a physical meaning. It was shown that bipolaron formation is favoured by larger values of $\alpha$ and by smaller values of $\eta$.

Intuitive arguments suggesting that the Fröhlich bipolaron is stabilised in going from $3 \mathrm{D}$ to $2 \mathrm{D}$ had been given, but the first quantitative analysis based on the Feynman path

integral was presented in Refs. (Verbist et al. , 1990, 1991). The conventional condition for bipolaron stability is $E_{\text {bip }} \leq 2 E_{\text {pol }}$, where $E_{\text {pol }}$ and $E_{\text {bip }}$ denote the ground-state energies 


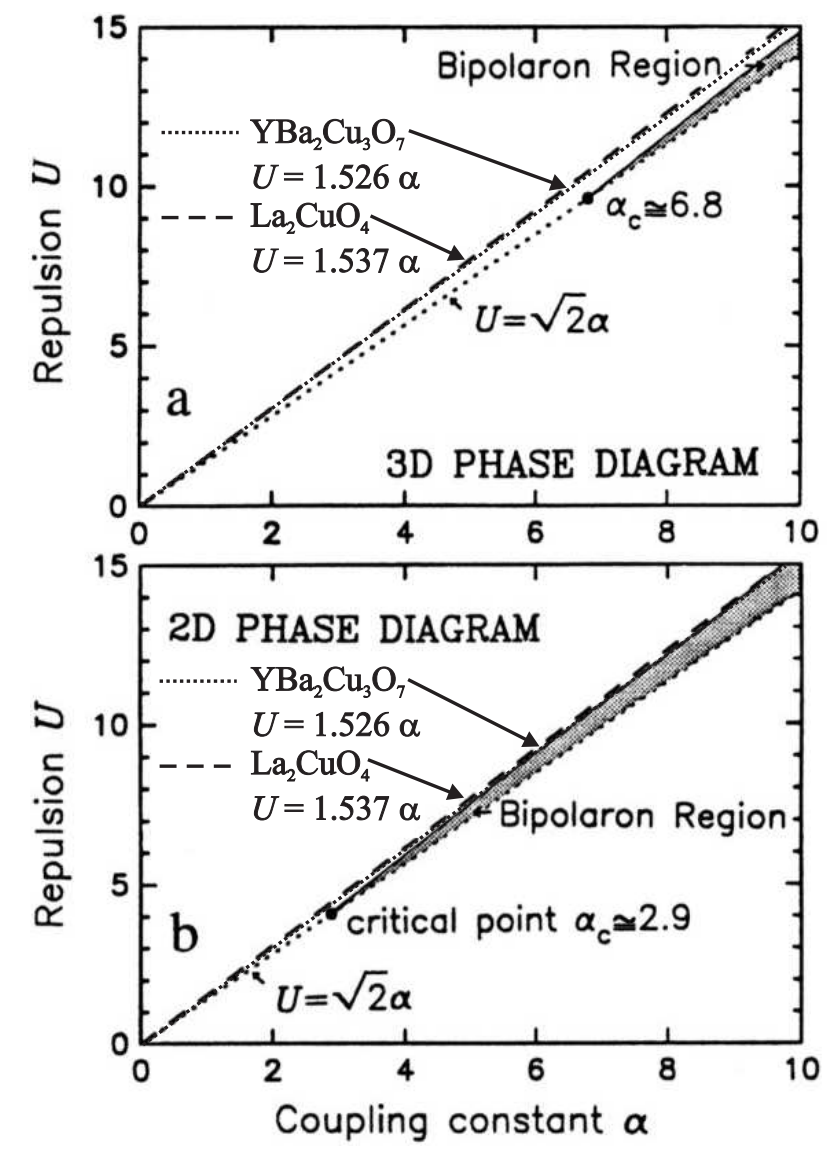

FIG. 14 The stability region for bipolaron formation in 3D (a) and in 2D (b). The dotted line $U=\sqrt{2} \alpha$ separates the physical region $(U \geq \sqrt{2} \alpha)$ from the non-physical $(U \leq \sqrt{2} \alpha)$. The shaded area is the stability region in physical space. The dashed (dotted) "characteristic line" $U=1.537 \alpha$ $(U=1.526 \alpha)$ is determined by $U=\sqrt{2} \alpha /\left(1-\varepsilon / \varepsilon_{0}\right)$ where we took the experimental values $\varepsilon=4$ and $\varepsilon_{0}=50$ for $\mathrm{La}_{2} \mathrm{CuO}_{4}\left(\varepsilon=4.7\right.$ (Genzel et al.), 1989) and $\varepsilon_{0}=64.7$ calculated using the experimental data of (Genzel et al., 1989; Kress et al., 1988) for $\mathrm{YBa}_{2} \mathrm{Cu}_{3} \mathrm{O}_{7}$ ). The critical points $\alpha_{c}=6.8$ for $3 \mathrm{D}$ and $\alpha_{c}=2.9$ for $2 \mathrm{D}$ are represented as full dots. (Reprinted with permission after Ref. (Verbist et al. , 1990). (C)1990 by Elsevier.)

of the polaron and bipolaron at rest, respectively. From this condition it follows that the Fröhlich bipolaron with zero spin is stable (given the effective Coulomb repulsion between electrons) if the electron-phonon coupling constant is larger than a certain critical value: $\alpha \geq \alpha_{c}$.

A "phase-diagram" for the two continuum polarons - bipolaron system is shown in Fig. 14 for 3D and for 2D. The Fröhlich coupling constant as high as 6.8 is needed to 
allow for bipolaron formation in 3D. The confinement of the bipolaron in two dimensions facilitates bipolaron formation at smaller $\alpha$. This can be shown using the PD-type scaling relation between the free energies $F$ in two dimensions $F_{2 D}(\alpha, U, \beta)$ and in three dimensions $F_{3 D}(\alpha, U, \beta)$ (Verbist et al. , 1990, 1991),

$$
F_{2 D}(\alpha, U, \beta)=\frac{2}{3} F_{3 D}\left(\frac{3 \pi}{4} \alpha, \frac{3 \pi}{4} U, \beta\right) .
$$

According to Eq. (257), the critical value of the coupling constant for bipolaron formation $\alpha_{c}$ turns out to scale with a factor $3 \pi / 4 \approx 2.36$ or $\alpha_{c}^{(2 D)}=\alpha_{c}^{(3 D)} / 2.36$. From Fig. 14b it is seen that bipolarons in 2D can be stable for $\alpha \geq 2.9$, a domain of coupling constants which is definitely realised in several solids. The "characteristic line" $U=1.526 \alpha$ for the material parameters of $\mathrm{YBa}_{2} \mathrm{Cu}_{3} \mathrm{O}_{7}$ enters the region of bipolaron stability in $2 \mathrm{D}$ at a value of $\alpha$ which is appreciably smaller than in the case of $\mathrm{La}_{2} \mathrm{CuO}_{4}$. This fact suggests $\mathrm{YBa}_{2} \mathrm{Cu}_{3} \mathrm{O}_{7}$ as a good candidate for the occurrence of stable Fröhlich bipolarons.

An analytical strong-coupling asymptotic expansion in inverse powers of the electronphonon coupling constant for the large bipolaron energy at $T=0$ was derived in (Smondyrev et al. , 1995)

$$
E_{3 D}(\alpha, u)=-\frac{2 \alpha^{2}}{3 \pi} A(u)-B(u)+O\left(\alpha^{-2}\right),
$$

where the coefficients are closed analytical functions of the ratio $u=U / \alpha$ :

$$
A(u)=4-2 \sqrt{2} u\left(1+\frac{u^{2}}{128}\right)^{3 / 2}+\frac{5}{8} u^{2}-\frac{u^{4}}{512}
$$

and for $B(u)$ see the above-cited paper. The scaling relation (257) allows one to find the bipolaron energy in two dimensions as well.

The stability of bipolarons has also been examined with the use of operator techniques with a variational approach (Bassani et al. , 1991). The bipolaron is bound if the electronphonon coupling constant $\alpha$ is larger than $\sim 6$ in three dimensions and larger than $\sim 2$ in two dimensions, provided the ratio $\eta=\varepsilon / \varepsilon_{0}$ is smaller than a critical value $\eta_{c}$ which depends on $\alpha$. The critical value $\eta_{c}$ is larger in the two-dimensional case than in the threedimensional one. The bipolaron radius is shown to be of the order of a few polaron radii. The results of (Bassani et al. , 1991) and (Verbist et al. , 1990, 1991) tend to qualitatively confirm each other. Furthermore, bipolaron states obtained in (Bassani et al. , 1991) under the assumption that the total linear momentum is conserved, have intrinsically high mobility. 
In the framework of the renewed interest in bipolaron theory after the discovery of high$\mathrm{T}_{c}$ superconductivity, an analysis of the optical absorption by large (Devreese et al. , 1995; Emin , 1993) and small (Alexandrov and Bratkovsky, 1999b) bipolarons was given.

\section{Discrete strong-coupling Fröhlich bipolaron}

The Holstein model is an extreme polaron model, and typically yields the highest possible values of the (bi)polaron mass in the strong coupling regime (except the case when the lattice vibrations are polarised along the hopping direction (Trugman et al. , 2001)). Many doped ionic lattices are characterized by poor screening of high-frequency optical phonons and they are more appropriately described by the finite-range Fröhlich EPI (Alexandrov, 1996). The unscreened Fröhlich EPI provides relatively light lattice polarons (IV.C) and also "superlight" small bipolarons, which are several orders of magnitude lighter than bipolarons in HHM (Alexandrov, 1996; (Alexandrov and Kornilovitch, 2002; Hague et al. 2, 2007a).

To illustrate the point let us consider a generic "Coulomb-Fröhlich" model (CFM) on a lattice, which explicitly includes the finite-range Coulomb repulsion and the strong longrange EPI (Alexandrov, 1996; Alexandrov and Kornilovitch , 2002). The implicitly present (infinite) Hubbard $U$ prohibits double occupancy and removes the need to distinguish the fermionic spin, if we are interested in the charge rather than spin excitations. Introducing spinless fermion annihilation operators $c_{\mathbf{n}}$ and phonon annihilation operators $d_{\mathbf{m}}$, the Hamiltonian of CFM is written in the real space representation as (Alexandrov and Kornilovitch, 2002)

$$
\begin{aligned}
H= & \sum_{\mathbf{n} \neq \mathbf{n}^{\prime}} T\left(\mathbf{n}-\mathbf{n}^{\prime}\right) c_{\mathbf{n}}^{\dagger} c_{\mathbf{n}^{\prime}}+\frac{1}{2} \sum_{\mathbf{n} \neq \mathbf{n}^{\prime}} V_{c}\left(\mathbf{n}-\mathbf{n}^{\prime}\right) c_{\mathbf{n}}^{\dagger} c_{\mathbf{n}} c_{\mathbf{n}^{\prime}}^{\dagger} c_{\mathbf{n}^{\prime}}+ \\
& \omega_{0} \sum_{\mathbf{n} \neq \mathbf{m}} g(\mathbf{m}-\mathbf{n})\left(\mathbf{e} \cdot \mathbf{e}_{\mathbf{m}-\mathbf{n}}\right) c_{\mathbf{n}}^{\dagger} c_{\mathbf{n}}\left(d_{\mathbf{m}}^{\dagger}+d_{\mathbf{m}}\right)+\omega_{0} \sum_{\mathbf{m}}\left(d_{\mathbf{m}}^{\dagger} d_{\mathbf{m}}+\frac{1}{2}\right),
\end{aligned}
$$

where $T(\mathbf{n})$ is the bare hopping integral in a rigid lattice.

If we are interested in the non- or near-adiabatic limit and the strong EPI, the kinetic energy is a perturbation. Then the model can be grossly simplified using the Lang-Firsov canonical transformation. In particular lattice structures like a staggered triangular ladder in Fig 16 the intersite lattice bipolarons tunnel already in the first order in $t(\mathbf{n})$. That allows us to average the transformed Hamiltonian over phonons to obtain its polaronic part 
as $H_{p}=H_{0}+H_{\text {pert }}$, where

$$
H_{0}=-E_{p} \sum_{\mathbf{n}} c_{\mathbf{n}}^{\dagger} c_{\mathbf{n}}+\frac{1}{2} \sum_{\mathbf{n} \neq \mathbf{n}^{\prime}} v\left(\mathbf{n}-\mathbf{n}^{\prime}\right) c_{\mathbf{n}}^{\dagger} c_{\mathbf{n}} c_{\mathbf{n}^{\prime}}^{\dagger} c_{\mathbf{n}^{\prime}},
$$

and

$$
H_{\text {pert }}=\sum_{\mathbf{n} \neq \mathbf{n}^{\prime}} t\left(\mathbf{n}-\mathbf{n}^{\prime}\right) c_{\mathbf{n}}^{\dagger} c_{\mathbf{n}^{\prime}} .
$$

is a perturbation. $E_{p}$ is the familiar polaron level shift,

$$
E_{p}=\omega \sum_{\mathbf{m}} g^{2}(\mathbf{m}-\mathbf{n})\left(\mathbf{e} \cdot \mathbf{e}_{\mathbf{m}-\mathbf{n}}\right)^{2},
$$

which is independent of $\mathbf{n}$. The polaron-polaron interaction is

$$
v\left(\mathbf{n}-\mathbf{n}^{\prime}\right)=V_{c}\left(\mathbf{n}-\mathbf{n}^{\prime}\right)-V_{p h}\left(\mathbf{n}-\mathbf{n}^{\prime}\right),
$$

where

$$
V_{p h}\left(\mathbf{n}-\mathbf{n}^{\prime}\right)=2 \omega_{0} \sum_{\mathbf{m}} g(\mathbf{m}-\mathbf{n}) g\left(\mathbf{m}-\mathbf{n}^{\prime}\right)\left(\mathbf{e} \cdot \mathbf{e}_{\mathbf{m}-\mathbf{n}}\right)\left(\mathbf{e} \cdot \mathbf{e}_{\mathbf{m}-\mathbf{n}^{\prime}}\right) .
$$

The transformed hopping integral is $t\left(\mathbf{n}-\mathbf{n}^{\prime}\right)=T\left(\mathbf{n}-\mathbf{n}^{\prime}\right) \exp \left[-g^{2}\left(\mathbf{n}-\mathbf{n}^{\prime}\right)\right]$ with

$$
\begin{aligned}
g^{2}\left(\mathbf{n}-\mathbf{n}^{\prime}\right)= & \sum_{\mathbf{m}} g(\mathbf{m}-\mathbf{n})\left(\mathbf{e} . \mathbf{e}_{\mathbf{m}-\mathbf{n}}\right) \times \\
& {\left[g(\mathbf{m}-\mathbf{n})\left(\mathbf{e} \cdot \mathbf{e}_{\mathbf{m}-\mathbf{n}}\right)-g\left(\mathbf{m}-\mathbf{n}^{\prime}\right)\left(\mathbf{e} \cdot \mathbf{e}_{\mathbf{m}-\mathbf{n}^{\prime}}\right)\right] }
\end{aligned}
$$

at $T \ll \omega_{0}$. The mass renormalization exponent can be expressed via $E_{p}$ and $V_{p h}$ as

$$
g^{2}\left(\mathbf{n}-\mathbf{n}^{\prime}\right)=\frac{1}{\omega_{0}}\left[E_{p}-\frac{1}{2} V_{p h}\left(\mathbf{n}-\mathbf{n}^{\prime}\right)\right] .
$$

When $V_{p h}$ exceeds $V_{c}$ the full interaction becomes negative and polarons form pairs. The real space representation allows us to elaborate more physics behind the lattice sums in $V_{p h}$ (Alexandrov and Kornilovitch , 2002). When a carrier (electron or hole) acts on an ion with a force $\mathbf{f}$, it displaces the ion by some vector $\mathbf{x}=\mathbf{f} / k$. Here $k$ is the ion's force constant. The total energy of the carrier-ion pair is $-\mathbf{f}^{2} /(2 k)$. This is precisely the summand in Eq.(261) expressed via dimensionless coupling constants. Now consider two carriers interacting with the same ion. The ion displacement is $\mathbf{x}=\left(\mathbf{f}_{1}+\mathbf{f}_{2}\right) / k$ and the energy is $-\mathbf{f}_{1}^{2} /(2 k)-\mathbf{f}_{2}^{2} /(2 k)-$ $\left(\mathbf{f}_{1} \cdot \mathbf{f}_{2}\right) / k$. Here the last term should be interpreted as an ion-mediated interaction between the two carriers. It depends on the scalar product of $\mathbf{f}_{1}$ and $\mathbf{f}_{2}$ and consequently on the relative positions of the carriers with respect to the ion. If the ion is an isotropic harmonic 
(a)

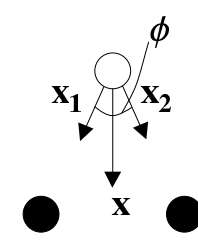

(b)

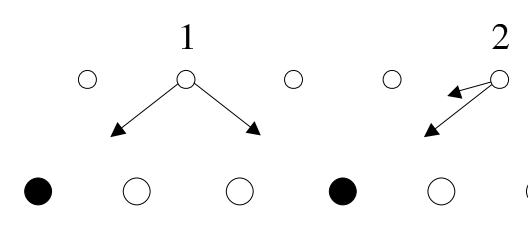

FIG. 15 Mechanism of the polaron-polaron interaction. (a) Together, two polarons (solid circles) deform the lattice more effectively than separately. An effective attraction occurs when the angle $\phi$ between $\mathbf{x}_{1}$ and $\mathbf{x}_{2}$ is less than $\pi / 2$. (b) A mixed situation: ion 1 results in repulsion between two polarons while ion 2 results in attraction. (After (Alexandrov and Kornilovitch, 2002))

oscillator, as we assume here, then the following simple rule applies. If the angle $\phi$ between $\mathbf{f}_{1}$ and $\mathbf{f}_{2}$ is less than $\pi / 2$ the polaron-polaron interaction will be attractive, otherwise it will be repulsive. In general, some ions will generate attraction, and some ions - repulsion between polarons.

The overall sign and magnitude of the interaction is given by the lattice sum in Eq.(263). One should note that according to Eq.(265) an attractive EPI reduces the polaron mass (and consequently the bipolaron mass), while repulsive EPI enhances the mass. Thus, the long-range EPI serves a double purpose. Firstly, it generates an additional inter-polaron attraction because the distant ions have a small angle $\phi$. This additional attraction helps to overcome the direct Coulomb repulsion between polarons. And secondly, the Fröhlich EPI makes lattice bipolarons lighter.

The many-particle ground state of $H_{0}$ depends on the sign of the polaron-polaron interaction, the carrier density, and the lattice structure. Following (Alexandrov and Kornilovitch, 2002), we consider the staggered ladder, Fig.16, assuming that all sites are isotropic twodimensional harmonic oscillators. For simplicity, we also adopt the nearest-neighbour approximation for both interactions, $g(\mathbf{l}) \equiv g, V_{c}(\mathbf{n}) \equiv V_{c}$, and for the hopping integrals, $T(\mathbf{m})=T(a)$ for $l=n=m=a$, and zero otherwise. Hereafter we set the lattice period 
$a=1$. There are four nearest neighbours in the ladder, $z=4$. The single-particle polaronic Hamiltonian takes the form

$$
\begin{aligned}
H_{p}= & -E_{p} \sum_{n}\left(c_{n}^{\dagger} c_{n}+p_{n}^{\dagger} p_{n}\right)+ \\
& \sum_{n}\left[t^{\prime}\left(c_{n+1}^{\dagger} c_{n}+p_{n+1}^{\dagger} p_{n}\right)+t\left(p_{n}^{\dagger} c_{n}+p_{n-1}^{\dagger} c_{n}\right)+\text { H.c. }\right],
\end{aligned}
$$

where $c_{n}$ and $p_{n}$ are polaron annihilation operators on the lower and upper legs of the ladder, respectively, Fig.16. Using Eqs.261]263]265) one obtains $E_{p}=4 g^{2} \omega_{0}, t^{\prime}=$ $T(a) \exp \left(-7 E_{p} / 8 \omega_{0}\right)$, and $t=T(a) \exp \left(-3 E_{p} / 4 \omega_{0}\right)$.

The Fourier transform of $H_{p}$ yields two overlapping polaron bands,

$$
E_{p}(k)=-E_{p}+2 t^{\prime} \cos (k) \pm 2 t \cos (k / 2)
$$

with the effective mass $m^{*}=2 /\left|4 t^{\prime} \pm t\right|$ near their edges.

Let us now place two polarons on the ladder. The nearest neighbour interaction is $v=V_{c}-E_{p} / 2$, if two polarons are on different legs of the ladder, and $v=V_{c}-E_{p} / 4$, if both polarons are on the same leg. The attractive interaction is provided via the displacement of the lattice sites, which are the common nearest neighbours to both polarons. There are two such nearest neighbours for the intersite bipolaron of type $A$ or $B$, Fig 16 , but there is only one common nearest neighbour for bipolaron $C$, Fig [16d. When $V_{c}>E_{p} / 2$, there are no bound states and the multi-polaron system is a one-dimensional Luttinger liquid. However, when $V_{c}<E_{p} / 2$ and consequently $v<0$, the two polarons are bound into an inter-site bipolaron of types $A$ or $B$.

Remarkably, bipolarons tunnel in the ladder already in the first order with respect to the single-polaron tunnelling amplitude. This case is different from both onsite bipolarons discussed above, and from intersite chain bipolarons of (Bonča and Trugman , 2001), where the intersite bipolaron tunnelling appeared in the second order in $t$ as for the on-site bipolarons. Indeed, the lowest-energy configurations $A$ and $B$ are degenerate. They are coupled by $H_{\text {pert }}$. Neglecting all higher-energy configurations, we can project the Hamiltonian onto the subspace containing $A, B$, and empty sites. The result of such a projection is the bipolaronic Hamiltonian

$$
H_{b}=\left(V_{c}-\frac{5}{2} E_{p}\right) \sum_{n}\left[A_{n}^{\dagger} A_{n}+B_{n}^{\dagger} B_{n}\right]-t^{\prime} \sum_{n}\left[B_{n}^{\dagger} A_{n}+B_{n-1}^{\dagger} A_{n}+\text { H.c. }\right],
$$


(a)

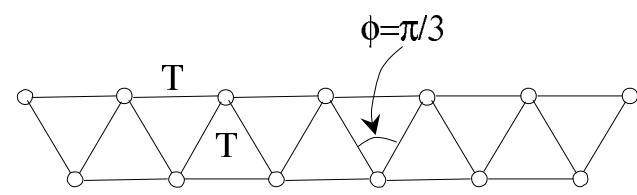

(b)

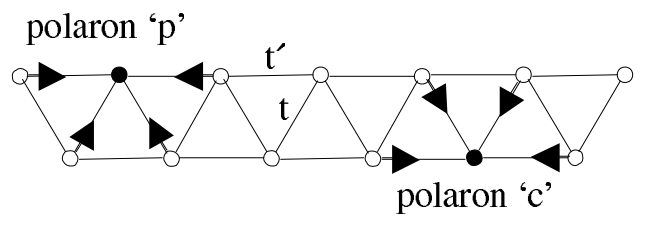

(c)

bipolaron A

polaron ' $\mathrm{c}$ '

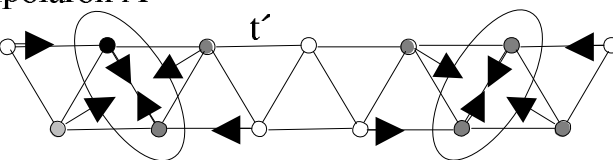

bipolaron B

(d)

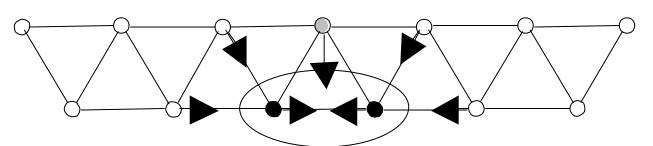

bipolaron C

FIG. 16 One-dimensional zig-zag ladder. (a) Initial ladder with the bare hopping amplitude $T(a)$. (b) Two types of polarons with their respective deformations. (c) Two degenerate bipolaron configurations $\mathrm{A}$ and $\mathrm{B}$ (here $t, t^{\prime}$ are renormalised (i.e. polaronic) hopping integrals). (d) A different bipolaron configuration, $\mathrm{C}$, whose energy is higher than that of $\mathrm{A}$ and $\mathrm{B}$.

where $A_{n}=c_{n} p_{n}$ and $B_{n}=p_{n} c_{n+1}$ are intersite bipolaron annihilation operators, and the bipolaron-bipolaron interaction is omitted. The Fourier transform of Eq.(268) yields two bipolaron bands,

$$
E_{2}(k)=V_{c}-\frac{5}{2} E_{p} \pm 2 t^{\prime} \cos (k / 2) .
$$

with a combined width $4\left|t^{\prime}\right|$. The bipolaron binding energy in zero order with respect to $t, t^{\prime}$ is $\Delta \equiv 2 E_{1}(0)-E_{2}(0)=E_{p} / 2-V_{c}$.

The bipolaron mass near the bottom of the lowest band, $m^{* *}=2 / t^{\prime}$, is

$$
m^{* *}=4 m^{*}\left[1+0.25 \exp \left(\frac{E_{p}}{8 \omega_{0}}\right)\right] .
$$

The numerical coefficient $1 / 8$ in the exponent ensures that $m^{* *}$ remains of the order of $m^{*}$ even at sufficiently large $E_{p}$ up to $E_{p} \approx 10 \omega_{0}$. This fact combined with a weaker renormalization of $m^{*}$ provides a superlight small bipolaron (Alexandrov, 1996; Alexandrov and Kornilovitch, 2002; Hague et al. ., 2007a). 


\section{E. Discrete all-coupling Fröhlich bipolaron}

The CFM model discussed above is analytically solvable in the strong-coupling nonadibatic $\left(\omega_{0} \gtrsim T(a)\right)$ limit using the Lang-Firsov transformation of the Hamiltonian, and projecting it on the inter-site pair Hilbert space (Alexandrov, 1996; Alexandrov and Kornilovitch, 2002). The theory has been extended to the whole parameter space using CTQMC technique for bipolarons (Hague et al. , 2007a, b). Refs. (Hague et al. , 2007a, b) simulated the CFM Hamiltonian on a staggered triangular ladder (1D), triangular (2D) and strongly anisotropic hexagonal (3D) lattices including triplet pairing. On such lattices, bipolarons are found to move with a crab like motion, Fig[16, which is distinct from the crawler motion found on cubic lattices (Alexandrov and Ranninger, 1981a). Such bipolarons are small but very light for a wide range of electron-phonon couplings and phonon frequencies. EPI has been modeled using the force function in the site-representation as in Eq.(194). Coulomb repulsion has been screened up to the first nearest neighbors, with on site repulsion $U$ and nearest-neighbor repulsion $V_{c}$. The dimensionless electron-phonon coupling constant $\lambda$ is defined as $\lambda=\sum_{\mathbf{m}} f_{\mathbf{m}}^{2}(0) / 2 M \omega^{2} z T(a)$ which is the ratio of the polaron binding energy to the kinetic energy of the free electron $z T(a)$, and the lattice constant is taken as $a=1$.

Extending the CTQMC algorithm to systems of two particles with strong EPI and Coulomb repulsion solved the bipolaron problem on different lattices from weak to strong coupling in a realistic parameter range where usual strong and weak-coupling limiting approximations fail. Figure 17 shows the ratio of the polaron to bipolaron masses on the staggered ladder as a function of effective coupling and phonon frequency for $V_{c}=0$. The bipolaron to polaron mass ratio is about 2 in the weak coupling regime $(\lambda \ll 1)$ as it should be for a large bipolaron, section VI.C. In the strong-coupling, large phonon frequency limit the mass ratio approaches 4 , in agreement with strong-coupling results, section VI.D. In a wide region of parameter space, the bipolaron/polaron mass ratio has been found between 2 and 4 and a bipolaron radius similar to the lattice spacing, Figs. 17. Thus the bipolaron is small and light at the same time. Taking into account additional intersite Coulomb repulsion $V_{c}$ does not change this conclusion. The bipolaron is stable for $V_{c}<4 T(a)$. As $V_{c}$ increases the bipolaron mass decreases but the radius remains small, at about 2 lattice spacings. Importantly, the absolute value of the small bipolaron mass is only about 4 times 


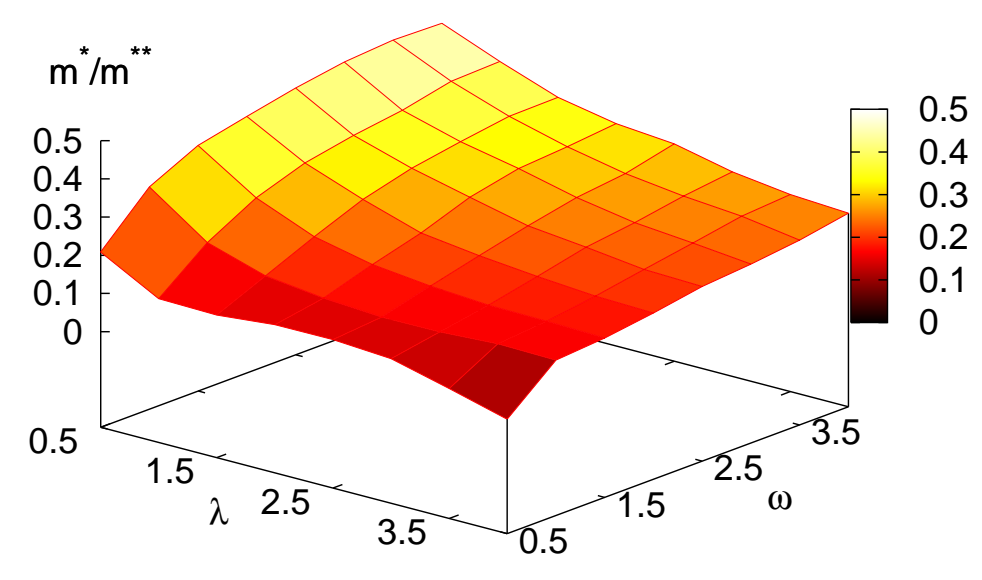

FIG. 17 Polaron to bipolaron mass ratio for a range of $\bar{\omega}=\omega_{0} / T(a)$ and $\lambda$ on the staggered ladder. Mobile small bipolarons are seen even in the adiabatic regime $\bar{\omega}=0.5$ for couplings $\lambda$ up to 2.5 (Reproduced from J. P. Hague et al., Phys. Rev. Lett 98, 037002 (2007), (c) American Physical Society, 2007).

of the bare electron mass $m_{0}$, for $\lambda=\omega / T(a)=1$ (see Fig. 17).

Simulations of the bipolaron on an infinite triangular lattice including exchanges and large on-site Hubbard repulsion $U=20 T(a)$ also lead to the bipolaron mass of about $6 m_{0 x y}$ and the bipolaron radius $R_{b p} \approx 2 a$ for a moderate coupling $\lambda=0.5$ and a large phonon frequency $\omega=T(a)$ (for the triangular lattice, $m_{0 x y}=1 / 3 a^{2} T(a)$ ). Finally, the bipolaron in a hexagonal lattice with out-of-plane hopping $T^{\prime}=T(a) / 3$ has also a light in-plane inverse mass, $m_{x y}^{* *} \approx 4.5 m_{0 x y}$ but a small size, $R_{b p} \approx 2.6 a$ for experimentally achievable values of the phonon frequency $\omega=T(a)=200 \mathrm{meV}$ and EPI, $\lambda=0.36$. Outof-plane $m_{z}^{* *} \approx 70 m_{0 z}$ is Holstein like, where $m_{0 z}=1 / 2 d^{2} T^{\prime}$, ( $d$ is the inter-plane spacing). When bipolarons are small and pairs do not overlap, the pairs can form a BEC at $T_{B E C}=$ $3.31\left(2 n_{B} / a^{2} \sqrt{3} d\right)^{2 / 3} /\left(m_{x y}^{2 / 3} m_{z}^{1 / 3}\right)$. If we choose realistic values for the lattice constants of 0.4 $\mathrm{nm}$ in the plane and $0.8 \mathrm{~nm}$ out of the plane, and allow the density of bosons to be $n_{B}=0.12$ per lattice site, which easily avoids overlap of pairs, then $T_{B E C} \approx 300 \mathrm{~K}$. 


\section{F. Polaronic exciton}

Finally let us briefly mention works on electron-hole bound states coupled with phonons. Such excitonic polaron states have been analysed by (Iadonisi and Bassani), 1983, 1984, 1987). The binding energies of excitonic states in interaction with LO phonons were computed using a phonon coherent state and applying a variational method (Iadonisi and Bassani , 1983). Incomplete relaxation of the lattice is found. The binding energies are larger than those obtained with static dielectric screening when the polaron radius is comparable to the exciton radius. Analysing the relative intensities of the one-phonon and zero-phonon lines for a number of semiconductors, (Iadonisi and Bassani , 1983) observed that the zero-phonon exciton states are generally much more probable than the phonon replicas.

The binding energy of a core exciton, which depends on the interaction of the conduction electron and of the core hole with the valence electrons via a Fröhlich-type coupling with the electron-hole pairs, in addition to the Coulomb attraction, was calculated within a functional variational method (Iadonisi and Bassani), 1984). When the exciton radius is comparable to the polaron radius, the static dielectric screening reduces and the core exciton binding energy increases. The excitonic-polaron effective mass renormalization was analysed by (Iadonisi and Bassani , 1984) using a variational numerical approach. Evidence for this renormalization is found from the experimental data on polariton dispersion in hexagonal $\mathrm{CdS}$ and in $\mathrm{CuCl}$.

The angular momentum as a constant of the motion of a Fröhlich polaron was introduced by (Evrard et al. , 1970). Representing the excitonic polaron problem in angular coordinates and solving it numerically, (Iadonisi et al. , 1989) showed that exciton states with various total angular momenta $L$ are differently affected by the interaction with LO phonons. However, when extending this approach to the bipolaron problem, the same authors found that the Fröhlich interaction is not strong enough to guarantee a stable bipolaron state at least in the case of cubic materials.

Very recently the polaronic exciton problem has been solved using the approximation-free DQMC technique (Burovski et al. , 2008). Numerically exact results for the wave function, ground-state energy, binding energy and effective mass of this quasiparticle were calculated, and the frequently used instantaneous approximation to the retarded interaction due to the 
exchange of phonons was critically analysed.

\section{CURRENT STATUS OF POLARONS AND OPEN PROBLEMS}

At present, the basic properties of single polarons are well understood theoretically, and to a large extent they are analytically under control at all coupling. It is remarkable how the Fröhlich continuum polaron, one of the simplest examples of a Quantum Field Theoretical problem, as it basically consists of a single fermion interacting with a scalar Bose field, has resisted full analytical solution at all coupling since $\sim 1950$, when its Hamiltonian was first written. Although a mechanism for the optical absorption of Fröhlich polarons was already proposed a long time ago (Devreese et al. , 1972a; Kartheuser et al. , 1969), some subtle characteristics were only clarified very recently (De Filippis et al. , 2006) by combining numerical DQMC studies (Mishchenko et al. , 2003) and improved analytical methods (Cataudella et al., 2007; Devreese and Klimin, 2006) (III.F). Of special interest are several sum rules derived for the optical conductivity spectra of arbitrary-coupling Fröhlich polarons (Devreese et al. ., 1977; Lemmens et al., 1973). A variety of magneto-optical and transportexperiments were successfully analysed with Fröhlich polaron theory (see e. g. (Devreese, 2004; Hodby et al., 1987; Peeters and Devreese, 1984; Peeters and Devreese , 1986) and references therein).

The charge carriers in a rich variety of systems of reduced dimension and dimensionality (submicron- and nanostructures including heterojunctions, quantum wells, quantum wires, quantum dots etc.) turn out to be Fröhlich polarons. Several scaling relations were derived (Peeters and Devreese, 1987), which connect polaron characteristics (the self-energy, the effective mass, the impedance and the mobility) in different dimensions. Quite generally confinement enhances EPI and the tendency to polaron formation. A new aspect of the polaron concept has been investigated for semiconductor structures at nanoscale: the excitonphonon states are not factorisable into an adiabatic product Ansatz, so that a non-adiabatic treatment is needed (Fomin et al., , 1998). The excitonic polarons in nanostructures lead to the existence of phonon replicas in the luminescence (Verzelen et al. , 2002). Experimental evidence of the enhanced phonon-assisted absorption due to effects of non-adiabaticity has been provided by the multi-phonon photoluminescence (PL) spectra observed under selective excitation in self-assembled InAs/GaAs quantum dots (García-Cristóbal et al., 1999) and by 
the photoluminescence excitation (PLE) measurements on single self-assembled InAs/GaAs (Lemaître et al., 2001) and InGaAs/GaAs (Zrenner et al., 2001) quantum dots. The polaron concept was also invoked for the explanation of the PLE measurements on self-organised $\mathrm{In}_{x} \mathrm{Ga}_{1-x} \mathrm{As} / \mathrm{GaAs}$ (Heitz et al., 2001) and CdSe/ZnSe (Woggon et al., 2003) quantum dots.

The Fröhlich polaron has led to many generalisations. The stability region of the Fröhlich large bipolaron is now firmly established (Bassani et al. , 1991; Verbist et al. , 1990, 1991)(VI.C). Here the surprise is double (cf. (Devreese et al. , 1995; Verbist et al. , 1991)): a) only in a very limited sector of the phase diagram (Coulomb repulsion versus $\alpha$ ) the bipolaron is stable, b) most traditional Fröhlich polaron materials (alkali halides and the like) lie completely outside (and "far" from) this bipolaron stability sector, but several cuprate superconductors lie very close and even inside this very restricted area of the stability diagram. The stability of a strong-coupling singlet bipolaron was studied in twoand three-dimensional parabolic quantum dots using the Landau-Pekar variational method (Mukhopadhyay et al. , 1996). It was shown that the confining potential of the quantum dot reduces the stability of the bipolaron. A theory of bipolaron states in a spherical parabolic potential well was further developed applying the Feynman variational principle. The basic parameters of the bipolaron ground state (the binding energy, the number of phonons in the bipolaron cloud, and the bipolaron radius were studied as a function of the radius of the potential well (Pokatilov et al., 1999). It was found that confinement can enhance the bipolaron binding energy, when the radius of a quantum dot is of the same order of magnitude as the polaron radius. A unified insight into the stability criterion for bipolaron formation in low-dimensionally confined media was provided by (Senger and Ercelebi, 2000) using an adiabatic variational method for a pair of electrons immersed in a reservoir of bulk LO phonons and confined within an anisotropic parabolic potential box. Bipolaron formation in a two-dimensional lattice with harmonic confinement, representing a simplified model for a quantum dot, was investigated by means of QMC (Hohenadler et al. , 2007a). This method treats all interactions exactly and takes into account quantum lattice fluctuations. Calculations of the bipolaron binding energy reveal that confinement opposes bipolaron formation for weak electron-phonon coupling but abets a bound state at intermediate to strong coupling. We also mention the exciton-polaron formation in nanostructures and quantum-light sources studied recently by QMC in lattice models with short- or long-range carrier-phonon interaction (see (Hohenadler et al. , 2007b), and references therein) 
The richness and profundity of Landau-Pekar's polaron concept is further illustrated by its extensions to discrete (lattice) polarons. Even the simplest two-site polaron model by Holstein (IV.A) proved to be very useful for a qualitative understanding of nontrivial features of the polaron problem, and for obtaining some novel analytical and semi-analytical results (see, for example, (Berciu, 2007; Han et al. ., 2002; Kudinov and Firsov, 1997)). The " $1 / \lambda$ " expansion technique based on the Lang-Firsov transformation (IV.B.1) and unbiased numerical analysis of the finite and infinite Holstein and Fröhlich models combining Lanczos diagonalisations of clusters, density matrix renormalisation group, cluster perturbation theory techniques, DMFT and different QMC algorithms, allowed for a description of properties of a single lattice polaron and a lattice bipolaron. The Lang-Firsov canonical transformation (Lang and Firsov, 1962) was proven particularly instrumental in calculation of different kinetic and optical coefficients, which can be represented as expansions in powers of the unrenormalised hopping integral $t(\mathbb{V})$. Sometimes it is possible to sum the expansion and get results, which are valid for arbitrary values of parameters providing the understanding of the crossover region from the Boltzmann kinetics to thermally activated hopping (Firsov, 2007).

Recent ED (Fehske and Trugman, 2007), CTQMC (Kornilovitch, 2007) and DQMC (Mishchenko and Nagaosa, 2007) techniques allow for determination of the ground-state and excited states of lattice polarons with arbitrary precision in the thermodynamic limit for any dimension and any type of lattices (IV.E.2). The spectral properties (e.g. photoemission), optical response and thermal transport, as well as the dynamics of polaron formation in the Holstein model have been numerically analysed for all EPI strengths and phonon frequencies, including the intermediate-coupling regime (see $\nabla$ and (Fehske and Trugman, 2007)). CTQMC methods have proven to be powerful and versatile tools providing unbiased results for the polaron properties in any lattices for any-range EPI, including Jahn-Teller polarons (IV.E). Combining the Lang-Firsov transformation and quantum Monte Carlo simulations allows for an exact sampling without autocorrelations, which proves to be an enormous advantage for small phonon frequencies or low temperatures (Hohenadler and von der Linden, 2007c).

Importantly, variational and numerical techniques confirmed that the Fröhlich and Holstein-Lang-Firsov theories are asymptotically exact in the weak, $\lambda \ll 1$, and strongcoupling, $\lambda \gg 1$, regimes, respectively, and the polaron formation represents a contin- 
uous crossover of the ground state (IV.E). The crossover is related to the exponential increase of the effective mass, and the band narrowing with a strongly suppressed electronic quasiparticle residue and the Drude weight, accompanied by an increase of the incoherent spectral weight $(\mathrm{V} . \mathrm{C})$. These features strongly depend on the phonon dispersion (Zoli , 1998), EPI radius (Alexandrov, 1996; Alexandrov and Kornilovitch , 1999; Fehske et al. , 2002; Perroni et al., 2005), lattice geometry (Hague et al. , 2006a) and are more pronounced in higher dimensions (Fehske et al. , 2002; Hague et al. , 2006a). Remarkably, the unscreened Fröhlich EPI provides relatively light lattice polarons (IV.C), which are several orders of magnitude lighter than the Holstein small polarons (Alexandrov, 1996; Alexandrov and Kornilovitch , 1999) at strong coupling. This classification of weak- and strong-coupling regimes still leaves room for continuum Fröhlich polarons of not only weak but also intermediate and - in the theoretical analysis - of strong coupling classified with respect to the Fröhlich electron-phonon coupling constant $\alpha$ (II.D) .

While the single polaron has been actively researched for a long time and is now well understood, the multi-polaron physics has gained particular attention in the last two decades. It has been found - unexpectedly for many researchers - that the Migdal-Eliashberg theory breaks down already at $\lambda \sim 1$ for any adiabatic ratio $\omega_{0} / E_{F}$. The effective parameter $\lambda \omega_{0} / E_{F}$ becomes large at $\lambda \gtrsim 1$ since the bandwidth is narrowed and the Fermi energy, $E_{F}$ is renormalised down exponentially (Alexandrov, 1983, 2001a). Extending the BCS theory towards the strong interaction between electrons and ion vibrations, a charged Bose gas of tightly bound on-site small bipolarons was predicted (Alexandrov and Ranninger, 1981a), with a further conclusion that the highest superconducting transition temperature is attained in the crossover region of EPI strength between the BCS-like polaronic and bipolaronic superconductivity (Alexandrov, 1983). Subsequent studies of the Holstein-Hubbard model found also two-site bipolarons (Aubry, 1995; Bonča et al. , 2000; La Magna and Pucci , 1997; Macridin et al. , 2004). Taking into account that many advanced materials with low density of free carriers and poor mobility are characterized by poor screening of high-frequency optical phonons, the Coulomb-Fröhlich lattice polaron model was introduced (Alexandrov, 1996; Alexandrov and Kornilovitch , 2002). The large Hubbard $U$ and intersite Coulomb repulsions and the unscreened Fröhlich EPI provide "superlight" but small intersite bipolarons (VI.D). More recent CTQMC simulations of intersite small bipolarons in the CoulombFröhlich model (Hague et al. , 2007a) have found such quasiparticles in a wide parameter 
range with achievable phonon frequencies and couplings. They could have a superconducting transition in excess of room temperature.

The many-body theory for polarons has been developed for extreme weak and strong coupling regimes. It became clear how - in the weak-coupling limit — this problem can be reduced to the study of the structure factor of a uniform electron gas (Lemmens et al., 1977). For strong coupling the problem is reduced to an interacting Bose gas of on-site (Alexandrov and Ranninger , 1981a) or intersite (Alexandrov, 1996; Alexandrov and Kornilovitch, 2002; Hague et al. , 2007a) small bipolarons in the dilute system.

While correlation effects in transport through metallic quantum dots with repulsive electron-electron interactions received considerable attention in the past, and continue to be the focus of intense investigations, much less has been known about a role of attractive correlations between small polarons mediated by EPI in molecular quantum dots (MQD) and nanowires. In the framework of the negative $U$ Hubbard model (Alexandrov et al. 2003) it has been found that the attractive electron correlations within the molecule could lead to a molecular switching effect where I-V characteristics have two branches with high and low current at the same bias voltage. The switching phenomenon has been also predicted by a theory of correlated polaron transport with a full account of both the Coulomb repulsion and EPI in MQD weakly coupled with electrodes (Alexandrov and Bratkovsky, 2003; Ermakov, 2000). Ref. (Alexandrov and Bratkovsky, 2003) has shown that while the phonon side-bands significantly modify the shape of hysteretic I-V curves in comparison with the negative- $U$ Hubbard model, switching remains robust. It shows up at sufficiently low temperatures when the effective interaction of polarons in MQD is attractive and the molecular level is multiply degenerate. Importantly, the switching has not been found in nonand two-fold degenerate MQDs (Alexandrov and Bratkovsky, 2003). When the polaronic energy shift is very large, the effective charging energy of molecules can become negative, favoring ground states with even numbers of electrons. Ref.(Koch et al., 2006) has shown that charge transport through such molecules is dominated by tunneling of bipolarons which coexists with single-electron cotunneling.

The conductance of deformable molecules with a local magnetic moment has been studied in the framework of a two-impurity Anderson model with positive and negative electronelectron interactions and in the two-impurity Anderson-Holstein model with a single phonon 
mode (Zitko and Bonca, 2006). It has been shown that the spin and charge Kondo effects can occur simultaneously at any coupling strength. At finite bandwidth and strong coupling the lattice effects lead to a renormalization of the effective Kondo exchange constants; nevertheless, universal spin and charge Kondo effects still occur.

The theory of dense polaronic systems in the intermediate regime remains highly cumbersome, in particular when EPI competes with strong electron correlations. The corresponding microscopic models contain (extended) Hubbard, Heisenberg or double-exchange terms, and maybe also a coupling to orbital degrees of freedom along with strong EPI, so that even numerical solutions with the same precision as in the dilute (bi)polaron case are often problematic. A number of ED, QMC, DMFT and combined numerical results give strong evidence that the tendency towards lattice polaron formation is enhanced in strongly correlated electron systems due to a narrowing of the electron band caused by strong correlations (see recent reviews by (Edwards, 2002; Fehske and Trugman , 2007; Hohenadler et al. , 2005b; Hohenadler and von der Linden, 2007c) for more details). Not only antiferromagnetic correlations enhance EPI, resulting in polaron formation for moderate coupling strength, but also EPI strongly enhances spin correlations (Capone and Ciuchi, 2002; Macridin et al. 2006). Some of these studies show that increasing carrier density could be accompanied by a dissociation of polarons leading to normal metallic behavior in the intermediate coupling adiabatic regime (Hohenadler et al. , 2005b) that is reminiscent of the "overcrowding" effect hypothesized by Mott (1995). On the other hand for parameters favoring small polarons no such density-driven crossover occurs in agreement with simple analytical arguments (Alexandrov, 2001a). Thorough investigations of these models will definitely be a great challenge in the near future. Here we have focused on the single and two-body problems leaving theories of strongly-correlated polarons and their applications to high-temperature superconductors and CMR oxides for future reviews (Alexandrov and Devreese , 2009).

Finally, exactly solvable models might give a rather limited, sometimes misleading, description of polarons in real systems. Qualitative inconsistencies can arise when coupling is assumed to be just to one phonon mode, often taken as dispersionless, and ad-hoc approximations for EPI matrix elements are applied. Moreover electronic nanoscale disorder and long-range strain fields can interweave with the microscopic mechanisms of polaronic transport (Phillips et al. , 2003). Hence ab-initio calculations of the phonon spectrum, EPI and polaron properties beyond the adiabatic Born-Oppenheimer approximation are required 
in many cases for which the theory and experiment can be compared in detail (Banacky), 2008; Shluger and Stoneham, 1993).

\section{Acknowledgments}

We have benefited from discussions with many colleagues. While writing this review, conversations with Alexander Andreev, Serge Aubry, Ivan Bozovic, Alex Bratkovsky, Peter Edwards, Janez Bonca, Holger Fehske, Jim Hague, Yurii Firsov, Martin Hohenadler, Viktor Kabanov, Pavel Kornilovitch, Wolfgang von der Linden, Peter Littlewood, Dragan Mihailovic, John Samson, Marshall Stoneham and other participants of the European Science Foundation workshop "Mott's Physics in Nanowires and Quantum Dots" (Cambridge, UK, 31 July-2 August, 2006) were especially helpful. We like to thank Vladimir Fomin for discussions during the preparation of this review, and to acknowledge discussions with Fons Brosens, Dieter Bimberg, Vittorio Cataudella, Paolo Calvani, Giulio De Filippis, Roger Evrard, Vladimir Gladilin, Giuseppe Iadonisi, Eddy Kartheuser, Serghei Klimin, Lucien Lemmens, Andrei Mishchenko, François Peeters and Jacques Tempere. This work was supported in part by EPSRC under grants No. EP/C518365/1 and No. EP/D07777X/1 (UK), IUAP, FWO-V project G.0435.03, the WOG WO.035.04N (Belgium) and the European Commission SANDiE Network of Excellence, contract No. NMP4-CT-2004-500101.

\section{References}

Adamowski, J., 1989, Phys. Rev. B 39, 3649.

Albrecht, U., and P. Leiderer, 1987, Europhys. Lett. 3, 705.

Alexandrov , A. S., 1983, Zh. Fiz. Khim. 57, 273 [Russ. J. Phys. Chem. 57, 167].

Alexandrov, A, 1991, Physica C (Amsterdam) 182, 327.

Alexandrov, A. S., 1992a, Phys. Rev. B 46, 2838.

Alexandrov, A. S., 1992b, Phys. Rev. B 46, 14932.

Alexandrov, A. S., 1996, Phys. Rev. B 53, 2863.

Alexandrov, A. S., 1998, in Proceedings of the International School of Physics "Enrico Fermi", Course CXXXVI, Varenna, 1997, "Models and Phenomenology for Conventional and High- 
Temperature Superconductivity", edited by G. Iadonisi, J. R. Schrieffer and M. L. Chiofalo (IOS Press, Amsterdam), 309.

Alexandrov, A. S., 2000, Phys. Rev. B 61, 12315.

Alexandrov, A. S., 2001a, Europhys. Lett. 56, 92.

Alexandrov, A. S., 2003, Theory of Superconductivity: from Weak to Strong Coupling (IoP, Bristol).

Alexandrov, A. S., and A. M. Bratkovsky, 1999a, Phys. Rev. Lett. 82, 141.

Alexandrov, A. S., and A. M. Bratkovsky, 1999b, J. Phys. Condens. Matter 48, L531.

Alexandrov, A. S., and A. M. Bratkovsky 2000, Phys. Rev. Lett. 84, 2043.

Alexandrov, A. S. and A. M. Bratkovsky, 2003, Phys. Rev. B 67, 235312.

Alexandrov, A. S., A. M. Bratkovsky, and R. S. Williams, 2003, Phys. Rev. B 67, 075301.

Alexandrov, A. S. and H. Capellmann, 1991, Phys. Rev. B 43, 2042.

Alexandrov, A. S., H. Capellmann, and U. Göbel,1992, Phys. Rev. B 46, 4374.

Alexandrov, A. S., and J. T. Devreese, 2009, Advances in Polaron Physics (Springer, Heidelberg).

Alexandrov, A. S., and V. V. Kabanov, 1986, Fiz. Tverd. Tela (St. Petersburg) 28, 1129 [Soviet Phys. Solid St. 28, 631 ].

Alexandrov, A. S., and V. V. Kabanov, 1996, Phys. Rev. B 54, 3655.

Alexandrov, A. S., V. V. Kabanov, and D. K. Ray, 1994a, Phys. Rev. B 49, 9915.

Alexandrov, A. S., V. V. Kabanov, and D. K. Ray 1994b, Physica C (Amsterdam) 224, 247.

Alexandrov, A. S., and P. E. Kornilovitch, 1999, Phys. Rev. Lett. 82, 807.

Alexandrov, A. S., and P. E. Kornilovitch 2002, J. Phys. Condens. Matter 14, 5337.

Alexandrov, A. S., and N. F. Mott, 1994, Rep. Prog. Phys. 57, 1197.

Alexandrov, A. S., and N. F. Mott, 1995, Polarons and Bipolarons ( World Scientific, Singapore).

Alexandrov, A., and J. Ranninger, 1981a, Phys. Rev. B 23, 1796.

Alexandrov, A., and J. Ranninger, 1981b, Phys. Rev. B 24, 1164.

Alexandrov, A. S., and J. Ranninger, 1992, Phys. Rev. B 45, 13109.

Alexandrov, A. S., and C. Sricheewin, 2000, EuroPhys. Lett. 51, 188.

Alexandrov, A. S., and B. Y. Yavidov, 2004, Phys. Rev. B 69, 073101.

Alexandrov, A. S., G. M. Zhao, H. Keller, B. Lorenz, Y. S. Wang, and C. W. Chu, 2001, Phys. Rev. B 64, 140404(R).

Allcock, G. R., 1956, Adv. Phys. 5, 412.

Allcock, G. R., 1963, in Polarons and Excitons, edited by C. G. Kuper and G. D. Whitfield (Oliver 
and Boyd, Edinburgh), 45.

Allen, P. B., and V. Perebeinos, 1999, Phys. Rev. B 60, 10747.

Alvermann, A., H. Fehske, and S. A. Trugman, 2008, Phys. Rev. B 78, 165106.

Anderson, P. W., 1975, Phys. Rev. Lett. 34, 953.

Anderson, P. W., 1997, The Theory of Superconductivity in the Cuprates (Princeton University Press, Princeton, NJ).

Appel, J., 1968, in Solid State Physics edited by F. Seitz, D. Turnbull, and H. Ehrenreich (Academic Press), 21.

Aubry, S., 1993, J. Phys. (France) IV Colloq. C2 3, 349.

Aubry, S., 1995, in Polarons and Bipolarons in High Tc Superconductors and related materials edited by E. K. H. Salje, A. S. Alexandrov and W. Y.Liang (Cambridge University Press, Cambridge), 271.

Aubry, S., 2007, in Polarons in Advanced Materials edited by A. S. Alexandrov (Canopus/Springer, Bristol), 311.

Banacky, P., 2008, J. Phys. Chem. Sol. 69, 2696.

Barisic, O. S., 2004, Phys. Rev. B 69, 064302.

Barisic, O. S., and S. Barisic, 2008, Eur. Phys. J. B 64, 1.

Baryakhtar, V. G., E. V. Zarochentsev, and E. P. Troitskaya, 1999, Theory of Adiabatic Potential and Atomic Properties of Simple Metals (Gordon and Breach, Amsterdam).

Bassani, F., M. Geddo, G. Iadonisi, and D. Ninno, 1991, Phys. Rev. B 43, 5296.

Becker, W., B. Gerlach, and H. Schliffke, 1983 Phys. Rev. B 28, 5735.

Bednorz, J. G., and K. A. Müller, 1988, Rev. Mod. Phys. 60, 585.

Belitz, D., and T. R. Kirkpatrick, 1994, Rev. Mod. Phys. 66, 261.

Benedetti, P., and R. Zeyher, 1998, Phys. Rev. B 58, 14320.

Beni, G., P. Pincus, and J. Kanamori, 1974, Phys. Rev. B 10, 1896.

Berciu, M., 2006a, Phys. Rev. Lett. 97, 036402.

Berciu, M., 2006b, Phys. Rev. B 74, 245104.

Berciu, M., 2007, Phys. Rev. B 75, 081101(R).

Berger, E., P. Valášek, and W. von der Linden, 1995, Phys. Rev. B 52, 4806.

Billinge, S. J. L., R. G. DiFrancesco, G. H. Kwei, J. J. Neumeier, and J. D. Thompson, 1996, Phys. Rev. Lett. 77, 715. 
Bogoliubov, N. N., 1950, Ukr. Math. Zh 2, 3.

Bogoliubov Jr., N. N., 1994, in Superconductivity and Strongly Correlated Electron Systems, ed. C.

Noce, A. Romano, and G. Scarpetta (World Scientific, Singapore), 107.

Bonča, J., T. Katrasnic, and S. A. Trugman, 2000, Phys. Rev. Lett. 84, 3153.

Bonča, J., and S. A. Trugman, 2001, Phys. Rev. B 64, 094507.

Bonča, J., S. A. Trugman, and I. Batistić, 1999, Phys. Rev. B 60, 1633.

Born, M., and R. Oppenheimer, 1927, Rev. Mod. Phys. 84, 457.

Böttger, H., and V. Bryksin, 1985, Hopping Conduction in Solids (Academie-Verlag, Berlin ).

Böttger, H., V. V. Bryksin, and T. Damker, 2007 in Polarons in Advanced Materials edited by A.

S. Alexandrov (Canopus/Springer, Bristol), 107.

Brosens, F., J. T. Devreese, and L. F. Lemmens, 1997a, Phys. Rev. E 55, 6795.

Brosens, F., J. T. Devreese, and L. F. Lemmens, 1997b, Phys. Rev. E 55, 227.

Brosens, F., J. T. Devreese, and L. F. Lemmens, 1998, Phys. Rev. E 58, 1634.

Brown, F. C. 1963, in Polarons and Excitons, ed. C. G. Kuper and G. D. Whitfield (Oliver and Boyd, Edinburgh), 323.

Brown, F. C., 1972, in Point Defects in Solids, edited by J. H. Crawford and L. M. Slifkin (Plenum, New York) 1, 537.

Bryksin, V. V., and Yu. A. Firsov, 1970, Sov. Phys. Sol. St. 12, 627.

Bryksin, V. V., and V. S. Voloshin, 1984, Fiz. Tverd. Tela (St Petersburg) 26, 2357[ Sov. Phys. Sol.

St. 26, 1429 (1984)].

Bryksin, V. V., and A. V. Gol'tsev, 1988, Fiz. Tverd. Tela (St Petersburg) 30, 1476[ Sov. Phys. Sol.

St. 30, 851 (1988)].

Burkov, A. A., and L. Balents , 2003, Phys. Rev. Lett. 91, 057202.

Burovski, E., H. Fehske, and A. S. Mishchenko, 2008, Phys. Rev. Lett. 101, 116403.

Bussmann-Holder, A., and A. R. Bishop, 1999, Phil. Mag, B 79, 119.

Bussmann-Holder, A., H. Keller, A. R. Bishop, A. Simon, R. Micnas, and K. A. Muller, 2005, Europhys. Lett. 72, 423.

Caldeira, A. O., and A. J. Leggett, 1981, Phys. Rev. Lett. 46, 211.

Castro Neto, A. H., and A. O. Caldeira, 1991, Phys. Rev. Lett. 67, 1960.

Calvani, P., 2001, Optical Properties of Polarons (Editrice Compositori, Bologna).

Calvani, P., M. Capizzi, S. Lupi, P. Maselli, A. Paolone, R. Roy, S. W. Cheong, W. Sadowski, and 
E. Walker, 1994, Solid State Commun. 91, 113.

Campbell, B. J., R. Osborn, D. N. Argyriou, L. Vasiliu-Doloc, J. F. Mitchell, S. K. Sinha, U. Ruett, C. D. Ling, Z. Islam, and J. W. Lynn, 2001, Phys. Rev. B 65, 014427.

Campbell, B. J., S. K. Sinha, R. Osborn, S. Rosenkranz, J. F. Mitchell D. N. Argyriou, L. VasiliuDoloc, O. H. Seeck, and J. W. Lynn, 2003, Phys. Rev. B 67, 020409.

Capone, M., and S. Ciuchi, 1997, Phys. Rev. B 65, 104409.

Capone, M., and S. Ciuchi, 2003, Phys. Rev. Lett. 91, 186405.

Capone, M., W. Stephan, and M. Grilli, 1997, Phys. Rev. B 56, 4484.

Cataudella, V., G. De Filippis, and G. Iadonisi, 1999, Eur. Phys. J. B 12, 17.

Cataudella, V., G. De Filippis, and C. A. Perroni, 2007, in Polarons in Advanced Materials edited by A. S. Alexandrov (Canopus/Springer, Bristol), 149.

Chakraverty, B. K., J. Ranninger, and D. Feinberg, 1998, Phys. Rev. Lett. 81, 433.

Chatterjee, J., and A. N. Das, 2000, Phys. Rev. B 61, 4592.

Chatterjee, J., A. N. Das, and P. Choudhury, 2003, Solid State Commun. 126, 113.

Cheng, J.-P., B. D. McCombe, J. M. Shi, F. M. Peeters, and J. T. Devreese, 1993, Phys. Rev. B 48, 7910 .

Ciuchi, S., F. de Pasquale, S. Fratini, and D. Feinberg, 1997, Phys. Rev. B 56, 4494.

De Filippis, G., V. Cataudella, V. Marigliano Ramaglia, C. A. Perroni, and D. Bercioux, 2003, Eur. Phys. J. B 36, 65.

De Filippis, G., V. Cataudella, A. S. Mishchenko, C. A. Perroni, and J. T. Devreese, 2006, Phys. Rev. Lett. 96, 136405.

De Mello, E. V. L., and J. Ranninger, 1997, Phys. Rev. B 55, 14872.

De Mello, E. V. L., and J. Ranninger, 1998a, Phys. Rev. B 58, 9098.

De Mello, E. V. L., and J. Ranninger, 1998b, Phys. Rev. B 58, 14625 .

De Mello, E. V. L., and J. Ranninger, 1999, Phys. Rev. B 59, 12135.

De Raedt, H., and A. Lagendijk, 1982, Phys. Rev. Lett. 49, 1522.

De Raedt, H., and A. Lagendijk, 1983, Phys. Rev. B 27, 6097.

De Raedt, H., and A. Lagendijk, 1984, Phys. Rev. B 30, 1671.

De Raedt, H., and A. Lagendijk, 1985, Phys. Rep. 127, 234.

Deppeler, A., and A. J. Millis, 2002, Phys. Rev. B 65, 224301.

Deville, G., A. Valdes, E. Y. Andrei, and F. I. B. Williams, 1984, Phys. Rev. Lett. 53, 588. 
Devreese, J., 1963, Bull. Soc. Belge de Phys., Ser. III, no. 4, 259.

Devreese, J. T. 1964 Contribution to the polaron theory, Ph.D. Thesis (KU Leuven).

Devreese, J. T., 1972 in Polarons in Ionic Crystals and Polar Semiconductors (North-Holland, Amsterdam), p. 83.

Devreese, J. T., 1996, in Encyclopedia of Applied Physics, edited by G. L. Trigg (VCH, Weinheim), volume 14,383 .

Devreese, J. T., 1998, in Proceedings of the International School of Physics "Enrico Fermi", Course CXXXVI, Varenna, 1997, "Models and Phenomenology for Conventional and High-Temperature Superconductivity", edited by G. Iadonisi, J. R. Schrieffer and M. L. Chiofalo (IOS Press, Amsterdam), 287.

Devreese, J. T., 2003, in Lectures on the Physics of Highly Correlated Electron Systems VII, edited by A. Avella and F. Mancini (AIP, Melville), 3.

Devreese, J. T., 2004, in Encyclopedia of Modern Optics, 5-volume set, edited by B. D. Guenther, D. G. Steel and L. Bayvel (Academic Press), 629.

Devreese, J. T., 2005, in Encyclopedia of Physics, Third Edition, edited by R. G. Lerner and L. Trigg (WILEY-VHC, Weinheim).

Devreese, J. T., 2006, in Proceedings of the "Enrico Fermi" Summer School, Course CLXI "Polarons in Bulk Materials and Systems with Reduced Dimensionality", Varenna, June 21 July 1, 2005, edited by G. Iadonisi, J. Ranninger, and G. De Filippis (IOS Press, Amsterdam), 27.

Devreese, J. T., 2007, J. Phys.: Condens. Matter 19, 255201.

Devreese, J. T., 2007, in Polarons in Advanced Materials, edited by A. S. Alexandrov (Canopus/Springer, Bristol,) 3 .

Devreese, J., and R. Evrard, 1963, Physica Status Solidi (b) 3, 2133.

Devreese, J. T., and R. Evrard, 1964, Phys. Letters 11, 278.

Devreese, J. T., and R. P. Evrard, 1965, Physica Status Solidi (b) 9, 403.

Devreese, J. T., and R. P. Evrard, 1966, Physics Letters 23, 196.

Devreese, J. T., and R. Evrard, 1968, in Proceedings of the British Ceramic Society 10, 151 [reprinted in Path Integrals and Their Applications in Quantum, Statistical, and Solid State Physics (NATO ASI Series B, Physics, vol. 34), edited by G. J. Papadopoulos and J. T. Devreese (Plenum, New York), 344 1977]. 
Devreese, J. T., J. De Sitter, and M. Goovaerts, 1971 Solid State Commun. 9, 1383.

Devreese, J. T., J. De Sitter, and M. Goovaerts, 1972 Phys. Rev. B 5, 2367.

Devreese, J. T., V. M. Fomin and E. P. Pokatilov, 2006, in Handbook of Semiconductor Nanostructures and Nanodevices, edited by A. A. Balandin and K. L. Wang, (American Scientific Publishers, Los Angeles), 4, 339.

Devreese, J., W. Huybrechts, and L. Lemmens, 1971 Phys. Stat. Sol. (b) 48, 77.

Devreese, J. T., and S.N. Klimin, 2006, unpublished.

Devreese, J. T., L. Lemmens, and J. Van Royen, 1977 Phys. Rev. B 15, 1212.

Devreese, J. T., and J. Tempere, 1998, Solid State Commun. 106, 309.

Devreese, J. T., G. Verbist, and F. M. Peeters, 1995 in Polarons and Bipolarons in High-Tc Superconductors and Related Materials, edited by E. K. H. Salje, A. S. Alexandrov, and W. Y. Liang(Cambridge University Press, Cambridge), 385.

Dogan, F., and F. Marsiglio, 2003 Phys. Rev. B 68, 165102.

Eagles, D. M. 1963, Phys. Rev. 130, 1381.

Eagles, D. M., 1966, Phys. Rev. 145, 645.

Eagles, D. M., 1969, Phys. Rev. 181, 1278.

Eagles, D. M., 1969, Phys. Rev. 186, 456.

Eagles, D. M., R. P. S. M. Lobo, and F. Gervais, 1995, Phys. Rev. B 52, 6440.

Edwards, D. M., 2002, Advan. Phys. 51, 1259.

Eliashberg, G. M., 1960, Zh. Eksp. Teor. Fiz. 38, 966 [Sov. Phys. JETP 11, 696 (1960)].

El Shawish, S., J. Bonča, L. C. Ku, and S. A. Trugman, 2003, Phys. Rev. B 67, 014301.

Emin, D., 1971, Ann. Phys. 64, 336.

Emin, D., 1973, Adv. Phys. 22, 57.

Emin, D., 1993, Phys. Rev. B 48, 13691.

Emin, D., and T. Holstein, 1976, Phys. Rev. Lett. 36, 323.

Entin-Wohlman, O., A. Aharony, Y. M. Galperin, V. I. Kozub, and V. Vinokur, 2005, Phys. Rev. Lett. 95, 086603.

Entin-Wohlman, O., A. G. Aronov, Y. Levinson, and Y. Imry, 1995, Phys. Rev. Lett. 75, 4094.

Ermakov, V. N., 1965, Physica E 8, 99.

Evrard, R., 1965, Phys. Lett. 14, 295.

Evrard, R., E. Kartheuser and J. Devreese, 1970, Phys. Stat. Sol. 41, 431-438. 
Fehske, H., D. Ihle, J. Loos, U. Trapper, and H. Buttner, 1994, Z. Phys. B 94, 91.

Fehske, H., H. Röder, G. Wellein, and A. Mistriotis, 1995, Phys. Rev. B 51, 16582.

Fehske, H., J. Loos, and G. Wellein, 1997, Z. Phys. B 104, 619.

Fehske, H., J. Loos, and G. Wellein, 2002, Phys. Rev. B 61, 8016.

Fehske, H., and S. A. Trugman, 2007, in Polarons in Advanced Materials edited by A. S. Alexandrov (Canopus/Springer, Bristol), 393.

Fehske, H., G. Wellein, G. Hager, A. Weiße, and A. R. Bishop, 2004, Phys. Rev. B 69, 165115.

Feynman, R. P., 1951, Phys. Rev. 84, 108.

Feynman, R. P., 1955, Phys. Rev. 97, 660.

Feynman, R. P., 1972, Statistical Mechanics (Benjamin).

Feynman, R. P., R. W. Hellwarth, C. K. Iddings, and P. M. Platzman, 1962, Phys. Rev. 127, 1004.

Finkenrath, H., N. Uhle, and W. Waidelich, 1969, Solid State Commun. 7, 11.

Firsov, Y. A., 1975, Polarons (Nauka, Moscow ), p. 553.

Firsov, Y. A., 2007, in Polarons in Advanced Materials edited by A. S. Alexandrov (Canopus/Springer, Bristol), 63.

Firsov, Y. A., V. V. Kabanov, E. K. Kudinov, and A. S. Alexandrov, 1999, Phys. Rev. B, 59, 12132.

Fisher, D. S., B. I. Halperin, and P. M. Platzman, 1979, Phys. Rev. Lett. 42, 798.

Fomin, V. M., V. N. Gladilin, J. T. Devreese, E. P. Pokatilov, S. N. Balaban, and S. N. Klimin, 1998, Phys. Rev. B 57, 2415.

Fonoberov, V. A., E. P. Pokatilov, V. M. Fomin, and J. T. Devreese, 2004, Phys. Rev. Lett. 92, 127402.

Fradkin, E., and J. E. Hirsch, 1983, Phys. Rev. B 27, 1680.

Frank, T., and M. Wagner, 1999, Phys. Rev. B 60, 3252.

Fratini, S., and S. Ciuchi, 2003, Phys. Rev. Lett. 91, 256403.

Fratini, S., and S. Ciuchi, 2005, Phys. Rev. B. 72, 235107.

Fratini, S., and S. Ciuchi, 2006, Phys. Rev. B. 74, 075101.

Fratini, S., and P. Quémerais, 2000, Eur. Phys. J. B 14, 99.

Friedman, L., and T. Holstein, 1963, Ann. Phys. 21, 494.

Fröhlich, H., 1937, Proc. R. Soc. (London) Ser. A 160, 230.

Fröhlich, H., 1954, Adv. Phys. 3, 325. 
Fröhlich, H., H. Pelzer, and S. Zienau, 1950, Phil. Mag. 41, 221.

Gadermaier, G., A. S. Alexandrov, V. V. Kabanov, P. Kusar, T. Mertelj, and X. Yao, C. Manzoni, G. Cerullo, and D. Mihailovic, 2009, arXiv:0902.1636.

García-Cristóbal, A., A. W. E. Minnaert, V. M. Fomin, J. T. Devreese, A. Y. Silov, J. E. M. Haverkort, and J. H. Wolter, 1999, Physica Status Solidi (b) 215, 331.

Genzel, L., A. Wittlin, M. Bauer, M. Cardona, E. Schönherr, and A. Simon, 1989, Phys. Rev. B 40, 2170.

Gerlach, B., and H. Löwen, 1991, Rev. Mod. Phys. 63, 63.

Gladilin, V. N., S. N. Klimin, V. M. Fomin, and J. T. Devreese, 2004, Physical Review B 69, 155325 (pages 5).

Gobel, U., A. S. Alexandrov, and H. Capellmann, 1994, Zeitschrift fur Physik B 96, 47.

Gogolin, A. A., 1982, Phys.Status Solidi B 109, 95.

Goovaerts, M. J., J. De Sitter, and J. T. Devreese, Phys. Rev. 7, 2639.

Gor'kov, L. P., and D. M. Chernikova, 1973, Pis'ma Zh. Eksp. Teor. Fiz. 18, 119 [JETP Lett. 18, $68,1973]$.

Götze, W., and P. Wolfle, 1972, Phys. Rev. B 6, 1226.

Grimes, C. C., and G. Adams, 1979, Phys. Rev. Lett. 42, 795.

Grosjean, C., 1957, in Lecture Notes on Solid State Physics, edited by D. Pines, (Princeton University Press, Princeton), chapter 8.

Gunnarsson, O., 1997, Rev. Mod. Phys. 69, 575.

Gurari, M., 1953, Phil. Mag. 44, 329.

Gurevich, V. L., I. G. Lang, and Yu. A. Firsov, Fiz.Tverd. Tela (St. Petersburg) 4, 1252 [Sov. Phys. Solid St. 4, 918 (1962)].

Gweon, G.-H., T. Sasagawa, S. Y. Zhou, J. Craf, H. Takagi, D. H. Lee, and A. Lanzara, 2004, Nature (London) 430, 187.

Hague, J. P., 2003, J. Phys. Condens. Matter 15, 2535.

Hague, J. P., P. E. Kornilovitch, A. S. Alexandrov, and J. H. Samson, 2006a, Phys. Rev. B 73, 054303.

Hague, J. P., P. E. Kornilovitch, J. H. Samson, and A. S. Alexandrov, 2007, Phys. Rev. Lett. 98, 037002 .

Hague, J. P., P. E. Kornilovitch, J. H. Samson, and A. S. Alexandrov, 2007, J. Phys.: Condens. 
Matter 19, 255214.

Hakioglu, T., and M. Y. Zhuravlev, 1998, Phys. Rev. B 58, 3777.

Han, R. S., Z. J. Lin, and K. L. Wang, 2002, Phys. Rev. B 65, 174303.

Hartinger, C., F. Mayr, J. Deisenhofer, A. Loidl, and T. Kopp, 2004, Physical Review B 69, 100403(R).

Heitz, R., H. Born, F. Guffarth, O. Stier, A. Schliwa, A. Hoffmann, and D. Bimberg, 2001, Phys. Rev. B 64, 241305(R).

Hellwege, K.-H. (editor in chief), 1982 Landolt-Börnstein Numerical Data and Functional Relationships in Science and Technology, New Series (Springer, New York), group III, Vol. 17b, p. 161.

Hendry, E., F. Wang, J. Shan, T. F. Heinz, and M. Bonn, 2004, Phys. Rev.B 69, 081101(R).

Hellwarth , R. W., and I Biaggio, 1999, Phys. Rev. B 60, 299.

Hendry, E., F. Wang, J. Shan, T. F. Heinz, and M. Bonn, 2004, Phys. Rev. B 69, 081101(R).

Hiramoto, H., and Y. Toyozawa, 1985, J. Phys. Soc. Japan 54, 245.

Hirsch, J. E., and E. Fradkin, 1982, Phys. Rev. Lett. 49, 402.

Hirsch, J. E., and E. Fradkin, 1983, Phys. Rev. B 27, 4302.

Hodby, J. W., G. P. Russell, F. M. Peeters, J. T. Devreese, and D. M. Larsen, 1987, Phys. Rev. Lett. 58, 1471.

Hohenadler, M., M. Aichhorn, and W. von der Linden, 2005, Phys. Rev. B 71, 014302.

Hohenadler, M., H. G. Evertz, and W. von der Linden, 2004, Phys. Rev. B 69, 024301 .

Hohenadler, M., and P. B. Littlewood, 2007a, Phys. Rev. B 76, 155122.

Hohenadler, M., P. B. Littlewood, and H. Fehske, 2007b, Phys. Rev. B 76, 184303.

Hohenadler, M., D. Neuber, W. von der Linden, G. Wellein, J. Loos, and H. Fehske 2005, Phys. Rev. B 71, 245111.

Hohenadler, M., G. Wellein, A. R. Bishop, A. Alvermann, and H. Fehske 2006, Phys. Rev. B 73, 245120.

Hohenadler, M., and W. von der Linden, 2007c, in Polarons in Advanced Materials edited by A.

S. Alexandrov (Canopus/Springer, Bristol), 463.

Hohenberg, P., and W Kohn, 1937, Proc. R. Soc. London A 136, 864.

Holstein, T., 1959, Ann. Phys. 8, 325.

Holstein, T., 1959, Ann. Phys. 8, 343. 
Howarth, D. J., and E. H. Sondheimer, 1953, Proc. R. Soc. (London) Ser. A 219, 53.

Huybrechts, W., and J. Devreese, 1973, Phys. Rev. B 8, 5754.

Huybrechts , W., and J. Devreese, 1975, Solid State Commun. 17, 401.

Iadonisi, G., and F. Bassani, 1983, Il Nuovo Cimento 2D, 1541-1560.

Iadonisi, G., and F. Bassani, 1984, Il Nuovo Cimento 3D, 408-420.

Iadonisi, G., and F. Bassani, 1987, Il Nuovo Cimento 9D, 703-714.

Iadonisi, G., G. Strinati and F. Bassani, 1989, Phys. Stat. Sol. (b) 153, 611-622.

Ioffe, A. F., 1957, Can. Phys. 26, 582.

Itoh, N., and A. M. Stoneham, 2001, Materials Modification by Electronic Excitation(Cambridge University Press, Cambridge).

Jackson, S. A., and P. M. Platzman, 1981, Phys. Rev. B 24, 499.

Jahn, H. A. and E. Teller, 1937, Phys. Rev. B 161, 220.

Jeckelmann, E., and S. R. White, 1998, Phys. Rev. B 57, 6376.

Jeckelmann, E., C. Zhang, and S. R. White, 1999, Phys. Rev. B 60, 7950.

Kabanov, V. V., and O. Y. Mashtakov, 1993, Phys. Rev. B 47, 6060.

Kadanoff, L. P., 1963, Phys. Rev. 130, 1364.

Kalosakas, G., S. Aubry, and G. P. Tsironis, 1998, Phys. Rev. B 58, 3094.

Kanamori, J., 1960, J. Appl. Phys. 31, S14.

Kartheuser, E., E. Evrard, and J. Devreese, 1969, Phys. Rev. Lett. 22, 94).

Kartheuser, E., 1972, in Polarons in Ionic Crystals and Polar Semiconductors, edited by J. T. Devreese (North-Holland, Amsterdam), 717.

Kashirina, N. I., V. D. Lakhno, and V. V. Sychyov, 2005, Phys. Rev. B 71, 134301).

Khasanov, R., D. G. Eshchenko, H. Luetkens, E. Morenzoni, T. Prokscha, A. Suter, N. Garifianov, M. Mali, J. Roos, K. Conder, and H. Keller, 2004, Phys. Rev. Lett. 92, 057602.

Klimin, S. N., F. Brosens, and J. T. Devreese, 2006, in preparation.

Klimin, S. N., V. M. Fomin, F. Brosens, and J. T. Devreese, 2004, Phys. Rev. B 69, 235324.

Klinger, M. I., 1963, Phys. Lett. 7, 102.

Klinger, M. I., 1979, Problems of polaron transport theory in semiconductors (Pergamon Press, Oxford).

Koch, J., M. E. Raikh, and F. von Oppen, 2006, Phys. Rev. Lett. 96, 056803.

Kochetov, E. A., S. P. Kuleshov, V. A. Mateev, and M. A. Smondyrev, 1977, Teor. Mat. Fiz. 30, 
183.

Kohn, W., and L. J. Sham, 1965, Phys. Rev. A 140, 1133.

Koller, W., D. Meyer, Y. Ono, and A. C. Hewson, 2004b, Europhys. Lett. 66, 559.

Kongeter, A., and M. Wagner, 1990, J. Chem. Phys. 92, 4003.

Kornilovitch, P. E., 1998, Phys. Rev. Lett. 81, 5382.

Kornilovitch, P. E., 1999, Phys. Rev. B. 60, 3237.

Kornilovitch, P. E., 2000, Phys. Rev. Lett. 84, 1551.

Kornilovitch, P. E., 2002, EuroPhys. Lett. 59, 735.

Kornilovitch, P. E., 2007, in Polarons in Advanced Materials edited by A. S. Alexandrov (Canopus/Springer, Bristol), 191.

Kornilovitch, P. E., and E. R. Pike, 1997, Phys. Rev. B. 55, R 8634.

Kornilovitch, P. E., and A. S. Alexandrov, 2004, Phys. Rev. B. 70, 224511.

Kress, W., U. Schröder, J. Prade, A. D. Kulkarni, and F. W. de Wette, 1988, Phys. Rev. B 38, 2906.

Krivoglaz, M. A., and S. I. Pekar, 1957, Bull. Acad. Sci. U.S.S.R. 21, 1, 13, 29.

Ku, L. C., S. A. Trugman, and J. Bonča, 2002, Phys. Rev. B 65, 174306.

Kudinov , E. K., and Yu. A. Firsov, 1965, Zh. Eksp. Teor. Fiz. 49, 867 [Sov. Phys. JETP 22, 603 (1965)].

Kudinov , E. K., and Yu. A. Firsov, 1966, Fiz. Tverd. Tela (St.-Petersburg) 8, 666 [Sov. Phys. Solid State 8, 536 (1966)].

Kudinov , E. K., and Yu. A. Firsov, 1997, Fiz. Tverd. Tela (St.-Petersburg) 39, 2159 [Phys. Solid State 39, 1930 (1997)].

Kudinov , E. K., D. N. Mirlin, and Yu. A. Firsov, 1969, Fiz. Tverd. Tela (St.-Petersburg) 11, 2789.

La Magna, A., and R. Pucci, 1997, Phys. Rev. B 55, 14886.

Landau, L. D., 1933, Physikalische Zeitschrift der Sowjetunion 3, 664.

Landau, L. D., and S. I. Pekar, 1948, Zh. Eksp. Teor. Fiz. 18, 419.

Lang, I. G., and Y. A. Firsov, 1962, Zh. Eksp. Teor. Fiz. 43, 1843 [Sov. Phys. JETP 16, 1301 (1962)].

Lang, I. G., and Y. A. Firsov, 1963, Zh. Eksp. Teor. Fiz. 45, 378 [Sov. Phys. JETP 18, 262 (1963)].

Lang, I. G., and Y. A. Firsov, 1968, Zh. Eksp. Teor. Fiz. 54, 826 [Sov. Phys. JETP 27, 443 (1968)].

Langreth, D. C., and L. P. Kadanoff, 1964, Phys. Rev. 133, A1070. 
Lanzara, A., P. V. Bogdanov, X. J. Zhou, S. A. Kellar, D. L. Feng, E. D. Lu, T. Yoshida, H. Eisaki, A. Fujimori, K. Kishio, J. I. Shimoyana, T. Noda, S. Uchida, Z. Hussain, and Z. X. Shen, 2001, Nature (London) 412, 510.

Lee, T. D., and D. Pines, 1952, Phys. Rev. 88, 960.

Lee, T. D., D. Pines, and F. Low, 1953, Phys. Rev. 90, 297.

Legget, A. J., 1980, J. Phys. (Paris) 41, C7.

Lemaître, A., A. D. Ashmore, J. J. Finley, D. J. Mowbray, M. S. Skolnick, M. Hopkinson, and T. F. Krauss, 2001, Phys. Rev. B 63, 161309(R).

Lemmens, L. F., F. Brosens, and D. J. T., 1977, Physica Status Solidi (b) 82, 439.

Lemmens, L. F., J. De Sitter, and J. T. Devreese, 1973, Phys. Rev. B 8, 2717.

Levinson, Y. B., E. I. Rashba, 1973, Rep. Prog. Phys. 36, 1499.

Loos, J., M. Hohenadler, and H. Fehske, 2006, J. Phys. Condens. Matter 18, 2453.

Low, F. E., and D. Pines, 1955, Phys. Rev. 98, 414.

Löwen, H., 1988, Phys. Rev. B 37, 8661.

Lupi, S., P. Maselli, M. Capizzi, P. Calvani, P. Giura, and P. Roy, 1999, Phys. Rev. Lett. 83, 4852.

Macridin, A., B. Moritz, M. Jarrell, and T. Maier, 2006, Phys. Rev. Lett. 97, 056402.

Macridin, A., G. A. Sawatzky, and M. Jarrell, 2004, Phys. Rev. B 69, 245111.

Mahan, G. D. 1972, in Polarons in Ionic Crystals and Polar Semiconductors ed. J. T. Devreese (North-Holland, Amsterdam ), p. 553.

Mahan, G. D., 1990, Many-Particle Physics (Kluwer/Plenum, New York)

Marsiglio, F., 1993, Phys. Lett. A 180, 280.

Marsiglio, F., 1995, Physica C 244, 21.

Maximov, E. G., D. Yu. Savrasov, and S. Yu. Savrasov, 1997, Uspechi Fiz. Nauk. 167, 353.

Meevasana, W., N. J. C. Ingle, D. H. Lu, J. R. Shi, F. Baumberger, K. M. Shen, W. S. Lee, T. Cuk, H. Eisaki, T. P. Devereaux, N. Nagaosa, J. Zaanen and Z. X. Shen, 2005, Phys. Rev. Lett. 96, 157003.

Mertelj, T., V. V. Kabanov, and D. Mihailović, 2005, Phys. Rev. Lett. 94, 147003.

Metropolis, N., A. W. Rosenbluth, M. N. Rosenbluth, A. M. Teller, and E. Teller, 1953, J. Chem. Phys. 21, 1087.

Micnas, R., J. Ranninger, and S. Robaszkiewicz, 1990, Rev. Mod. Phys. 62, 113.

Migdal, A. B., 1958, Zh. Eksp. Teor. Fiz. 34, 1438 [Sov. Phys. JETP 7, 996 (1958)]. 
Mihailović, D., C. M. Foster, K. Voss, and A. J. Heeger, 1990, Phys. Rev. B 42, 7989.

Mihailović, D., and V. V. Kabanov, 2001, Phys. Rev. B 63, 054505.

Millis, A. J., P. B. Littlewood, and B. I. Shraiman, 1995, Phys. Rev. Lett. 74, 5144.

Millis, A. J., B. I. Shraiman, and R. Mueller, 1996, Phys. Rev. Lett. 77, 175.

Mishchenko, A. S., and N. Nagaosa, 2004, Phys. Rev. Lett. 93, 036402.

Mishchenko, A. S., and N. Nagaosa, 2006, Phys. Rev. B 73, 092502.

Mishchenko, A. S., and N. Nagaosa, 2007, in Polarons in Advanced Materials, edited by A. S. Alexandrov (Canopus/Springer, Bristol), 503.

Mishchenko, A. S., N. V. Prokof'ev, A. Sakamoto, and B. V. Svistunov, 1990, Phys. Rev. B 62, 6317.

Mishchenko, A. S., N. Nagaosa, N. V. Prokof'ev, A. Sakamoto, and B. V. Svistunov, 2003, Phys. Rev. Lett. 91, 236401.

Mitra, T. K., A. Chatterjee, and S. Mukhopadhyay, 1987, Phys. Reports 153, 91.

Miura, N., H. Nojiri, P. Pfeffer, and W. Zawadzki, 1997, Phys. Rev. B 55, 13598.

Miyake, S. J., 1975, J. Phys. Soc. Jap. 38, 181.

Mori, H., 1965, Prog. Theor. Phys. 33, 423.

Mott, N. F., and E. A. Davis, 1979, Electronic processes in non-crystalline materials, (2nd edn. Oxford University Press, Oxford).

Mukhomorov, V. K., 1982, Fiz. Tekh. Poluprovodn. (St.-Peterburg) 16, 1095 [Sov. Phys. Semicond. 16, 700 (1982)].

Mukhopadhyay, S., and A. Chatterjee, 1996, J. Phys. Condens. Matter 8, 4017.

Müller, K. A., 2000, Physica C 341, 11.

Nagels, P., M. Denayer, and J. Devreese, 1963 Solid State Commun. 1, 35.

Nozieres , P., and S. Schmitt-Rink, 1985, J. Low Temp. Phys. 59, 195.

Osaka, Y., 1959, Prog. Theor. Phys. 22, 437.

Osaka, Y., 1961, Prog. Theor. Phys. 25, 517.

Paci, P., M. Capone, E. Cappelluti, S. Ciuchi, C. Grimaldi, and L. Pietronero, 2005, Phys. Rev. Lett. 94, 036406.

Peeters, F. M., and J. T. Devreese, 1982, Phys. Status Solidi B 112, 219.

Peeters, F. M., and J. T. Devreese, 1983a, Phys. Rev. B 28, 6051.

Peeters, F. M., and J. T. Devreese, 1983b, Physica Status Solidi (b) 115, 539. 
Peeters, F. M., and J. T. Devreese, 1984, in Solid State Physics, edited by F. Seitz and D. Turnbull, (Academic Press, New York), 38, 81.

Peeters, F. M., and J. T. Devreese, 1985, Phys. Rev. B 31, 6826.

Peeters, F. M., and J. T. Devreese, 1986, Phys. Rev. B 34, 7246.

Peeters, F. M., and J. T. Devreese, 1987, Phys. Rev. B 36, 4442.

Peeters, F. M., W. Xiaoguang, and J. T., Devreese, 1986, Phys. Rev. B 33, 3926.

Peierls, R. E., 1933, Z. Phys. 80, 763.

Pekar, S. I. 1946, Zh. Eksp. Teor. Fiz. 16, 335.

Pekar, S. I. 1951, Research in Electron Theory of Crystals, (US AEC Transl. AEC-tr-555, Russian edition 1951, German edition 1954).

Perroni, . A., V. Cataudella, and G. De Filippis2004, J. Phys. Condens. Matter 16, 1593.

Perroni, C. A., V. Cataudella G. De Filippis, and V. M. Ramaglia, 2005, Phys. Rev. B 71, 054301.

Phillips, J. C., A. R. Bishop, and A. Saxena, 2003, Rep. Prog. Phys. 66, 2111.

Platzman, P. M., 1963, in Polarons and Excitons, edited by C. G. Kuper and G. D. Whitfield, (Oliver and Boyd, Edinburgh), 123.

Pokatilov, E. P., V. M. Fomin, J. T. Devreese, S. N. Balaban, and S. N. Klimin, 1999, J. Phys. Condens. Matter 11, 9033.

Pokatilov, E. P., S. N. Klimin, V. M. Fomin, J. T. Devreese, and F. W. Wise, 2002, Phys. Rev. B 65, 075316 .

Prokof'ev, N. V., and B. V. Svistunov, 1998, Phys. Rev. Lett. 81, 2514.

Proville, L., and S. Aubry, 1998, Physica D 113, 307.

Proville, L., and S. Aubry, 1999, Eur. Phys. J. B 11, 41.

Radovic, Z., N. Bozovic, and I. Bozovic, 2008, Phys. Rev. B 77, 092508.

Ranninger, J., and U. Thibblin, 1992, Phys. Rev. B 45, 7730.

Rashba, E. I., 1957, Optika i spektroskopia 2, 75.

Rashba, E. I., 2005, in Encyclopedia of Condensed Matter Physics edited by G. F. Bassani, G. L. Liedi, and P. Wyder (Elsevier, Amsterdam), p. 347.

Reik, H. G., 1963, Sol. St. Commun. 1, 67.

Reik, H. G., 1972 in Polarons in Ionic Crystals and Polar Semiconductors, edited by J. T. Devreese (North-Holland, Amsterdam), p. 679.

Reznik, D., L. Pintschovius, M. Ito, S. Iikubo, M. Sato, H. Goka, M. Fujita, K. Yamada, G. D. Gu, 
and J. M. Tranquada, 2006, Nature 440, 1170.

Rietschel, H., L. Pintschovius, and W. Reichardt, 1989, Physica C (Amsterdam) 162, 1705.

Robaszkiewicz, S., R. Micnas, and K. A. Chao, 1981, Phys. Rev. B 23, 1447.

Roder, H., J. Zang, and A. R. Bishop, 1996, Phys. Rev. Lett. 76, 1356.

Romero, A. H., D. W. Brown, and K. Linderberg, 1998, J. Chem. Phys. 109, 6540.

Romero, A. H., D. W. Brown, and K. Lindenberg 1999, Phys. Rev. B 59, 13728.

Ruscher, C. H., K. R. Dey, T. Debnath, I. Horn, R. Glaum, and A. Hussain 2008, J. Solid State Chemistry 181, 90.

Salje, E. K. H., A. S. Alexandrov, and W. Y. Liang (eds.), 1995, Polarons and Bipolarons in High-Tc Superconductors and Related Materials (Cambridge University Press, Cambridge).

Salomaa, M. M., and G. A. Williams, 1981, Phys. Rev. Lett. 47, 1730.

Schubert, G., G. Wellein, A. Weiße, A. Alvermann, and H. Fehske, 2005, Phys. Rev. B 72, 104304.

Schultz, T. D., 1962, in Polarons and Excitons edited by C. G. Kuper and G. D. Whitfield (Oliver and Boyd, Edinburgh), 71.

Sendyka, T. R., W. Dmowski, T. Egami, N. Seiji, H. Yamauchi, and S. Tanaka 1995, Phys. Rev. B 51, 6747 .

Senger, R. T., and A. Ercelebi, 2000, Euro. Phys. J. B 16, 439.

Sewell, G. L., 1958, Phil. Mag. 3, 1361.

Shi, J. M., F. M. Peeters, and J. T. Devreese, 1993, Phys. Rev. B 48, 5202.

Shikin, V. B., 1978, JETP Lett. 27, 39.

Shikin, V. B., and Y. P. Monarkha, 1973, Zh. Eksp. Teor. Fiz. 65, 751 [Sov. Phys.—JETP 38, 373, 1973].

Shim, H., P. Chaudhari, G. Logvenov, and I. Bozovic, 2008, Phys. Rev. Lett. 101, 247004

Shluger, A. L., and A. M. Stoneham, 1993, J. Phys. Condens. Matter 1, 3049.

Sil, S., 1999, J. Phys.: Condens. Matter 11, 8879.

Silvera, I. F., 2001, Bull. Am. Phys. Soc. 46, 1016.

Smondyrev, M. A., and V. M. Fomin, 1994, in Polarons and Applications (Proceedings in nonlinear science), edited by V. D. Lakhno (John Wiley \& Sons, Chichester), 13.

Smondyrev, M. A., J. T. Devreese, and F. M. Peeters, 1995, Phys. Rev. B 51, 15008.

Spencer, P. E., J. H. Samson, P. E. Kornilovitch, and A. S. Alexandrov, 1995, Phys. Rev. B 71, 184310. 
Stephan, W., 1996, Phys. Rev. B 54, 8981.

Street, R. A., and N. F. Mott, 1975 Phys. Rev. Lett. 1293, 1975.

Su, W. P., J. R. Schrieffer, and A. J. Heeger, 1979, Phys. Rev. Lett. 42, 1698.

Sumi, H., 1974, J. Phys. Soc. Jpn. 36, 770.

Suprun, S. G., and B. Y. Moizhes, 1982, Fiz. Tverd. Tela (S.-Peterburg) 24, 1571.

Swain, S., 1973, J. Phys. A 6, 192.

Takada, Y., 2000, Phys. Rev. B 61, 8631.

Tempere, J., and J. T. Devreese, 2001 Phys. Rev. B 64, 104504.

Tempere, J., S. N. Klimin, I. F. Silvera, and J. T. Devreese, 2003a, Eur. Phys. J. B 32, 329.

Tempere, J., I. F. Silvera, and J. T. Devreese, 2001, Phys. Rev. Lett. 87, 275301.

Tempere, J., I. F. Silvera, and J. T. Devreese, 2003b, Physical Review B 67(3), 035402 (pages 8).

Thornber, K. K., and R. P. Feynman, 1970, Phys. Rev. B 1, 4099.

Titantah, J. T., C. Pierleoni, and S. Ciuchi, 2001, Phys. Rev. Lett. 87, 206406.

Tokura, Y., 2000, Colossal Magnetoresistance Oxides(Gordon and Breach, New York).

Tomonaga, S., 1947, Prog. Theoret. Phys. 2, 6.

Trugman, S. A., J. Bonča, and L. C. Ku, 2001, Int. J. Mod. Phys. B 15, 2707.

Tyablikov, S. V., 1952, Zh. Eksp. Teor. Fiz 23, 381.

van Mechelen, J. L., D. van der Marel, C. Grimaldi, A. B. Kuzmenko, N. P. Armitage, N. Reyren, H. Hagemann and I. I. Mazin, 2008, Phys. Rev. Lett. 100, 226403.

Verzelen, O., R. Ferreira, and G. Bastard, 2002, Phys. Rev. Lett. 88, 146803.

Verbist, G., F. M. Peeters, and J. T. Devreese, 1990, Sol. St. Commun. 76, 1005.

Verbist, G., F. M. Peeters, and J. T. Devreese, 1991, Phys. Rev. B 43, 2712.

Vinetskii, V. L. 1961, Zh. Eksp. Teor. Fiz. 40, 1459. [Sov. Phys. JETP 13, 1023].

Vinetskii, V. L., and M. S. Giterman, 1957, Zh. Eksp. Teor. Fiz. 33, 730.

Volodin, A. P., M. S. Khaikin, and V. S. Edelman, 1977, JETP Lett. 26, 543.

Weiße, A., G. Wellein, A. Alvermann, and H. Fehske, 2006, Rev. Mod. Phys. 78, 275.

Wellein, G., H. Röder, and H. Fehske, 1996, Phys. Rev. B 53, 9666.

Wellein, G., and H. Fehske 1997, Phys. Rev. B 56, 4513.

Wellein, G., and H. Fehske, 1998, Phys. Rev. B 58, 6208.

Woggon, U., D. Miller, F. Kalina, B. Gerlach, D. Kayser, K. Leonardi, D. Kayser and D. Hommel, 2003, Phys. Rev. B 67, 045204. 
Xiaoguang, W., F. M. Peeters, and J. T. Devreese, 1985, Phys. Rev. B 31, 3420.

Yamashita, J., and T. Kurosawa, 1958, J. Phys. Chem. Solids 5, 34.

Yunoki, S., A. Moreo, and E. Dagotto, 1998, Phys. Rev. Lett. 81, 5612.

Zamboni, R., A. J. Pal, and C. Taliani, 1989, Solid State Commun. 70, 813.

Zhang, C., E. Jeckelmann, and S. R. White, 1999, Phys. Rev. B 60, 14092.

Zhao, G. M., and D. E. Morris, 1995, Phys. Rev. B 51, 16487.

Zhao, G.-M., K. Conder, H. Keller, and K. A. Müller, 1996, Nature (London) 381, 676.

Zhao, G.-M., M. B. Hunt, H. Keller, and K. A. Müller, 1997, Nature (London) 385, 236.

Hang, Z., 1988, Phys. Rev. B 37, 7419.

Zheng, H., and M. Avignon, 2003, Phys. Rev. B 68, 024301.

Zitko, R., and J. Bonca, 2006, Phys. Rev. B 74, 224411.

Zoli, M., 1998, Phys. Rev. B 57, 10555.

Zoli, M., 1998, Phys. Rev. B 61, 14523.

Zoli, M., 2007, in Polarons in Advanced Materials edited by A. S. Alexandrov (Canopus/Springer, Bristol), 231.

Zoli, M., and A. N. Das, 2004, J. Phys.: Condens. Matter 16, 3597.

Zrenner, A., F. Findeis, M. Baier, M. Bichler, and G. Abstreiter, 2001, Physica B 298, 239. 